\title{
Cognitive Influences on Sensory Processing OF VisUAL Motion
}

\author{
Dissertation \\ zur Erlangung des Doktorgrades \\ der Mathematisch-Naturwissenschaftlichen Fakultäten \\ der Georg-August Universität zu Göttingen
}

\author{
vorgelegt von \\ Dipl. Psych., M. Sc. \\ Steffen Katzner \\ aus Weil im Schönbuch
}

Göttingen 2006 
D 7

Referent: Prof. Dr. Stefan Treue

Korreferent: Prof. Dr. Julia Fischer

Tag der mündlichen Prüfung: 04.07.2006 


\section{Acknowledgments}

Given the span of research questions this dissertation addresses, I would have found it impossible to conduct any of these studies without abundant expert assistance. I therefore wish to thank Stefan Treue for supervising this work. Throughout these years, I have enormously benefited from being exposed to his way of thinking. I also wish to acknowledge the special efforts of Christoph Nothdurft and Fred Wolf in providing me with their constructive criticism and helpful suggestions, and the kind support of Julia Fischer in evaluating this dissertation.

Many people have contributed to the different studies presented here. First of all, I would like to thank Dirk Prüsse for providing expert knowledge on all questions of animal care-taking, and for his continuous availability and commitment. In the few cases where Dirk was indisposed, Pinar Boyraz and Daniel Kaping took over the daily routine, which I am grateful for. I also appreciate the help of Ralf Brockhausen and Sabine Stuber for computer-related expertise and administrative support throughout the years.

I have benefited from discussions with Thilo Womelsdorf, Florian Pieper, Tzvetomir Tzvetanov, Alexander Gail, and Christine Tillmann. Also, I was fortunate to have Florian's technical support and assistance concerning the electrophysiological part of this work. Special Thanks go to Robert Niebergall for making the lab an enjoyable place, being the only one who knows how it feels to put a volley into the top right corner.

I am also happy to thank those kind people who have always encouraged me and have seen so little of me during the course of this work: my Dad, Uschi, Anja, Jürgen, Julia, Craig.

Last, and most, thank you Laura. 


\section{Contents}

1 Introduction 1

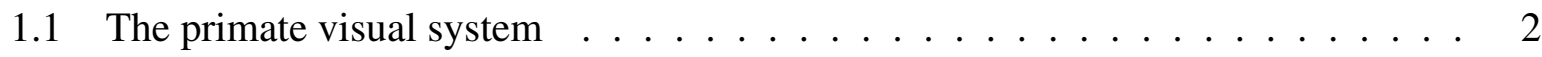

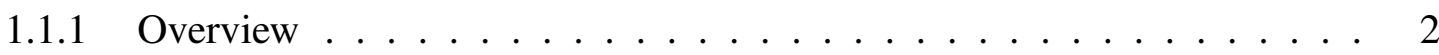

1.1.2 Properties of the motion-sensitive middle temporal area (MT) . . . . 3

1.2 Cognitive influences on sensory visual processing . . . . . . . . . 4

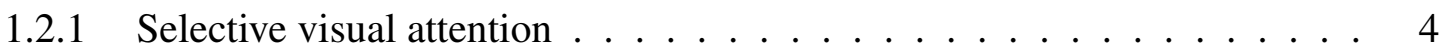

1.2.2 Effects of attention on visual motion processing . . . . . . . . 7

2 Original articles and manuscripts 9

2.1 Object-based attention in area MT . . . . . . . . . . . . . 10

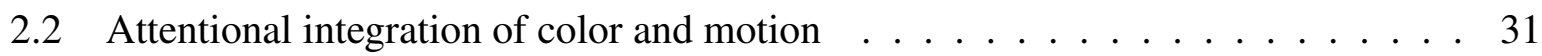

2.3 Benefits of signal anticipation $\ldots \ldots \ldots \ldots \ldots \ldots$

2.4 Exogenous cueing of motion processing . . . . . . . . . . . . . 64

2.5 Time course of shifting visual attention $\ldots \ldots \ldots \ldots \ldots$

$\begin{array}{lll}3 & \text { Summary } & 77\end{array}$

$\begin{array}{ll}\text { Bibliography } & 79\end{array}$

$\begin{array}{lr}\text { Curriculum Vitae } & 85\end{array}$ 


\section{Chapter 1}

\section{Introduction}

The current work represents a compilation of three scientific articles and two manuscripts that have been prepared for submission, all of them addressing the same central question: How do cognitive factors influence the sensory processing of visual information? While four of these studies examine the role of selective attention in visual processing, one focuses on the influence of stimulus predictability on perceptual sensitivity.

The model system we are studying in our research group is the highly developed ability to process visual motion information in higher primates. To investigate cognitive influences on the processing of visual motion I have used two different, but complementary approaches: On the one hand, I have recorded extracellular activity from individual, motion-sensitive neurons in the visual cortex of awake behaving macaque monkeys, on the other hand, I have performed psychophysical experiments with human observers.

This chapter is divided into two parts. Part 1 provides an overview of the primate visual system, emphasizing the processing of visual motion in the middle temporal area (MT) of extrastriate cortex. Part 2 describes how sensory information in the visual system is shaped by cognitive influences from higher-level areas in the brain. Here, the focus will mainly be on selective attention, which is one of the most prominent top-down mechanisms modulating sensory processing.

The main part of this work consists of original research articles, whose specific objectives will briefly be introduced in separate sections preceding each manuscript. 


\subsection{The primate visual system}

\subsubsection{Overview}

Light entering our eyes is first processed in the retina, where photoreceptors transduce electromagnetic radiation within the visible spectrum into electrical signals. These signals are transferred, through a network of interneurons (horizontal, bipolar, and amacrine cells), to the retinal ganglion cells. Each ganglion cell responds to stimulation of a restricted area of the retina, constituting that cell's receptive field (RF). Presenting a spot of light inside the RF of a ganglion cell causes a change in the neuronal response, whereas light placed anywhere outside this region has no influence on the cell's activity. In general, RFs are a universal property of visual neurons and represent an important concept for understanding visual information processing. In the primate visual system, the majority of retinal ganglion cells can be assigned to one of two functional categories: M ganglion cells have large RFs and respond to transient visual events, e.g., to rapidly moving objects, whereas $\mathrm{P}$ ganglion cells have smaller RFs and are sensitive to different wavelengths, thereby contributing to the perception of color. This functional separation is maintained along the central visual pathway and has led to the influential concept of anatomically different and functionally separate processing streams, each of them performing a distinct analysis of the visual information (Ungerleider and Mishkin, 1982).

The axons of the ganglion cells leaving the retina form the bilateral optic nerves that converge at the optic chiasm. Here, the optic nerve from each eye branches into two segments: fibers originating from the nasal part of the retinas cross to the opposite hemisphere (contralateral fibers), while fibers covering the temporal part of the retinas remain on the same side (ipsilateral fibers). Within the chiasm, contralateral fibers from one eye join ipsilateral fibers from the other eye, resulting in the left and right part of our visual world being represented in the right and left hemispheres of our brain, respectively. These re-arranged ganglion cell axons form the optic tract, projecting to subcortical brain structures. The major subcortical targets for the optic tract consist of the pretectum, the superior colliculus (SC) and the lateral geniculate nucleus (LGN) of the thalamus. While the SC controls saccadic eye movements, the pretectum is involved in the regulation of pupillary reflexes. However, with $90 \%$ of the retinal axons terminating in the LGN, this structure represents the major relay station for input to the visual cortex.

The projection of ganglion cells to the LGN is highly ordered, such that each LGN contains a retinotopic representation of the contralateral visual field, with neighboring cells coding information from neighboring regions on the retina. The LGN itself consists of six layers of cell bodies differing in size. The two most ventral layers contain large cells and are therefore termed magnocellular layers. In contrast, the remaining 4 layers contain smaller cells and are referred to 
as parvocellular layers. Whereas the P-type retinal ganglion cells provide input to the parvocellular layers, the $\mathrm{M}$ ganglion cells project to the magnocellular layers. Like the retinal ganglion cells, LGN cells have circular RFs. Recently, another population of cells, the koniocellular, or $\mathrm{K}$ cells has been identified within the LGN. While K cells also have distinct patterns of connections to the visual cortex their functional role is less clear (Hendry and Reid, 2000). Neurons in magnocellular and parvocellular layers send their axons to different layers in the primary visual cortex (V1), strictly maintaining the functional separation beginning in the retina. V1 is composed of six layers, and the RFs of its neurons are fundamentally different from those of retinal ganglion and LGN cells. Instead of small spots of light, these neurons prefer gradients in illumination, such as produced by bars, edges, and borders. Moreover, neurons in V1 are selective for orientation and direction, and they also encode information about the color of a stimulus.

Beyond V1 and along the hierarchy of cortical visual processing, RFs change in at least two fundamental ways. First, they progressively increase in size, covering larger and larger portions of the visual field. Second, lower-tier visual areas preferentially process simple stimulus attributes, like oriented lines (V1), while higher visual cortical areas respond optimally to increasingly complex stimulus properties, such as specific patterns of motion (MT/MST, Saito et al., 1986), objects of a certain shape (IT, Kreiman et al., 2006), or even individual faces (temporal lobe along the STS, Tsao et al., 2006). According to their functional specialization, the cortical visual areas can be broadly grouped into two major processing streams (Ungerleider and Mishkin, 1982; Felleman and Van Essen, 1991). Areas constituting the ventral pathway (V1, V2, V4, TEO, IT) are specialized for the processing of color, texture, shape, and object identity. In contrast, areas forming the dorsal pathway (V1, V2, V3, MT/MST) are involved in processing information about motion, binocular disparity, and spatial relations.

\subsubsection{Properties of the motion-sensitive middle temporal area (MT)}

Since all our experiments examine cognitive influences on the processing of visual motion, this section shortly reviews relevant properties of the middle temporal area (MT) in the dorsal processing stream (for more detailed reviews see Born and Bradley, 2005; Britten, 2004). MT is a heavily myelinated structure, located on the posterior bank of the superior temporal sulcus (STS) (Van Essen et al., 1981). The major input to MT comes directly from V1. In addition to V1 input, MT receives ascending signals from $\mathrm{V} 2, \mathrm{~V} 3$, and the lateral subdivision of the pulvinar complex. MT projects to a variety of other cortical areas in the STS (FST, MST), but also to parietal lobe (VIP, LIP, 7a), and frontal lobe areas (SEF, FEF). In addition, there are descending connections to the brainstem and the midbrain (SC). Like V1, MT is retinotopically organized with each hemisphere containing a map of the contralateral visual field (Gattass and Gross, 1981). Neurons 
representing the fovea are found in the lateral part of MT, while neurons whose RFs cover eccentric parts of the visual field are located more medially. The lower visual hemifield has a larger representation residing in the posterior part, the upper visual hemifield in the anterior part of MT. About $90 \%$ of neurons in area MT are highly selective for processing of motion direction and speed, while they are poorly suited to signal the color or the shape of a moving stimulus. Moreover, MT also exhibits a columnar organization, with neurons of similar direction preferences forming elongated clusters perpendicular to the surface of the cortex (Albright, 1984; DeAngelis and Newsome, 1999). The receptive fields of MT neurons are well-defined, being $\sim 10$ times larger than those in area V1. As a rule of thumb, the diameter of an MT receptive field is related to its eccentricity by a factor of 0.8 (Maunsell and Van Essen, 1983).

Direction preferences of individual MT neurons can be quantified by plotting a cell's response strength as a function of the direction of motion of the stimulus inside the RF. The resulting tuning curve has a bell-shaped form which is well described by a Gaussian model function. The direction of motion evoking the strongest response from a given neuron is called the preferred direction, while the opposite direction is commonly referred to as null- or anti-preferred direction. An individual MT neuron's selectivity is described by the bandwidth of the tuning curve, which has been reported to be, on average, $\sim 50-60^{\circ}$ half-width at half-height (Maunsell and Van Essen, 1983). Speed-tuning properties of MT neurons have been investigated less systematically. In general, neurons in area MT are bandpass-tuned with a preference for medium speeds, and decreases in response to either slower or faster speeds (Maunsell and Van Essen, 1983). Several lines of evidence have established a major role of area MT in the perception of visual motion (for a review see, Parker and Newsome, 1998). First, measures for sensitivity of individual neurons in MT can account for the behavioral performance in a direction discrimination task (Britten et al., 1992). Second, lesioning area MT results in considerable elevations of discrimination thresholds for the direction of moving stimuli with no impairment in the processing of stimulus contrast (Newsome and Pare, 1988). Third, micro-stimulation of MT neurons biases the monkey's report of perceived direction of motion towards the direction preferentially coded by the stimulated neurons (Salzman et al., 1990).

\subsection{Cognitive influences on sensory visual processing}

\subsubsection{Selective visual attention}

At any given moment, our visual system is confronted with much more information than we can fully process. To cope with this deluge, powerful mechanisms have evolved enabling our visual system to emphasize currently relevant over less important aspects in our environment. 
Such mechanisms are already present in the very first steps of visual processing. For example, visual information is enormously condensed in the retina, where 130 million photoreceptors converge onto only 1 million retinal ganglion cells. The circuitry in the retina performs major computations, such as enhancement of contrast between adjacent stimuli by lateral inhibition. This is only one of many examples demonstrating that vision is an active process during which the most important information is given priority in processing. In addition to such bottom-up mechanisms, top-down cognitive influences also strongly modulate the processing of sensory information. The most prominent of these cognitive influences is selective attention.

In the context of this work, the term attention refers to a selective modulation of sensory processing according to behavioral relevance. In everyday life, attention is tightly coupled to eye movements, such that relevant information is typically foveated. To disentangle mechanisms related to eye movements from those important for attention, laboratory studies generally investigate covert visual attention, i.e., to attentional improvements of visual processing in the absence of eye movements. Previous studies have described at least three different types of covert visual attention. Spatial attention refers to the ability to attend to a restricted location in the visual field. Processing of sensory information presented at the attended location is enhanced compared to processing of information outside the spatial focus of attention (Posner, 1980; Eriksen and St. James, 1986). Feature-based attention, on the other hand, describes a mechanism by which attending to a particular feature (i.e., the color red, or upward motion) enhances processing of the attended feature across the entire visual scene, independent of the spatial focus of attention (Treue and Martínez-Trujillo, 1999; Sàenz et al., 2002). Finally, object-based attention means that attention can also be directed to perceptual groups, or objects, and that all features belonging to the same object receive enhanced processing, even if they are irrelevant (O'Craven et al., 1999; Blaser et al., 2000). These three different types of visual attention are subject of the experiments presented in chapter 2 .

The neuronal correlates of spatial attention are well documented. It has been shown by a variety of brain activity measures that attention to a certain spatial location enhances activity of those neurons processing the sensory information at the attended location (e.g., Woldorff et al., 1997; Luck et al., 1997; Brefczynski and DeYoe, 1999). Modulation of neuronal activity by spatial attention can be observed in all areas of visual cortex, and a few studies have even reported effects of spatial attention in the LGN (O'Connor et al., 2002; McAlonan et al., 2006). While it has previously been assumed that the effects of attention increase with increasingly higher visual areas (Maunsell, 2004), a recent study combining functional magnetic resonance imaging (fMRI) and magnetoencephalography (MEG) has provided evidence that this is not necessarily the case (Hopf et al., 2006b). Rather, it seems that the effects of attention are strongest in those visual areas whose RF properties best match the spatial scale of the attended stimulus. In addition to 
the neuronal enhancement at the spatial focus of attention, computational models incorporating the anatomy and physiology of the primate visual system (Tsotsos et al., 1995) have predicted a suppressive zone around the focus of attention. While such center-surround organization of the spatial focus of attention has been observed in behavioral studies before (Cutzu and Tsotsos, 2003), a neuronal correlate for an inhibitory surround has been demonstrated only recently (Hopf et al., 2006a). Furthermore, recent studies argue that the spatial focus of attention can be split, such that attention can simultaneously be directed to multiple regions in the visual field (Awh and Pashler, 2000; McMains and Somers, 2004). Together, these studies illustrate some of the currently debated issues in the context of spatial attention.

In comparison to spatial attention, feature-based attention has been studied considerably less (for a recent review see Maunsell and Treue, 2006). Knowledge of the mechanisms of featurebased attention has been gained from visual search paradigms, in which an observer is instructed to find a predefined target item embedded in a display of distractors. In visual search tasks, feature-based attention might be particularly useful, since it enhances processing of the attended feature across the whole display. Using combined EEG/MEG recordings, Hopf et al. (2004) have demonstrated that feature-based attention can in fact be used to highlight potential target items, based on which spatial attention can then be oriented. Recently, evidence for such a mechanism has also been found for individual neurons in area V4. Here, single-unit activity was modulated if the attended target feature matched the preferences of the recorded neurons, independent of the spatial location (Bichot et al., 2005). Furthermore, several studies have examined the effects of feature-based attention for different dimensions. For instance, neuronal responses of orientationselective neurons in area V4 were modulated when attention was directed to the color versus the orientation of a stimulus outside the RF of the recorded neuron (McAdams and Maunsell, 2000). Similarly, a number of fMRI studies have demonstrated that neuronal activity is modulated selectively in areas that are specialized for processing of the attended feature (Corbetta et al., 1990; Watanabe et al., 1998; Beauchamp et al., 1997). In addition, some of the most important insights into mechanisms of feature-based attention have been obtained from single-unit studies in area MT. Section 1.2.2 describes these findings in more detail.

An increasing number of studies have demonstrated that attention can also be directed to whole objects. In general, they revealed that the overall organization of visual features into perceptual groups, or objects, can strongly influence the allocation of attention. Evidence for object-based attention comes from psychophysical experiments, in which subjects were cued to direct attention to a spatially defined part of one of two presented objects. Here, target-detection performance is better for stimuli at an unexpected location within the cued object, compared to stimuli at an equidistant location within the uncued object (Egly et al., 1994). This finding has been supported by a recent fMRI study reporting enhancement of lower-tier visual cortical activ- 
ity at retinotopic representations of both the cued and the uncued locations within the attended object, whereas retinotopic representations of locations in the uncued object were not modulated by attention (Müller and Kleinschmidt, 2003). Moreover, multi-unit recordings from primary visual cortex show that firing rates of V1 neurons can depend on whether the stimulus inside their RF is part of a relevant or irrelevant object (Roelfsema et al., 1998). Mechanisms of objectbased attention have also been examined in paradigms in which two objects were superimposed, such that spatial location by itself could not be used to orient attention. Typically, it has been found that performance was better for judging two properties of the same object than for two properties belonging to different objects ('same-object-advantage') (Duncan, 1984; Blaser et al., 2000). These findings already suggest that attention directed to a single feature of an object transfers to other features of the same object. In line with this prediction, a fMRI study (O'Craven et al., 1999) has demonstrated that attending to a single attribute of a visual object enhances the neuronal representation of another feature belonging to the same object, even if this feature is irrelevant.

\subsubsection{Effects of attention on visual motion processing}

For a long time, visual motion processing has been viewed as taking place pre-attentively. Meanwhile, a number of studies have revealed strong influences of both spatial and feature-based attention on sensory processing of visual motion. In a seminal paper, Treue and Maunsell (1999) demonstrated effects of spatial attention on firing rates of individual MT neurons by comparing responses to identical visual stimulation under different attentional conditions. In these experiments, two stimuli were presented, one of them inside the RF of the neuron under study, the other one outside. When the animal direction spatial attention to the stimulus inside versus outside the $\mathrm{RF}$, firing rates increased by $\sim 20 \%$.

In addition to these spatial effects of attention, subsequent studies have also demonstrated feature-based effects in area MT. Here, the spatial focus of attention was always directed to the stimulus outside the RF of the recorded neuron, and a preferred-direction stimulus was presented inside. Attending to the preferred versus null direction of the stimulus outside increased firing rates by $\sim 13 \%$ (Treue and Martínez-Trujillo, 1999). Similar effects of feature-based attention for processing of motion have also been reported in a recent fMRI study (Sàenz et al., 2002).

For both spatial and feature-based attention, the underlying mechanism is an increase in response gain at the single-neuron level, which often leads to a multiplicative scaling of the neuron's tuning curve without changes in its bandwidth (Treue and Martínez-Trujillo, 1999). Furthermore, it has been shown that feature-based attention to a particular direction of motion only increases responses of neurons preferring the attended direction, while it decreases responses of 
neurons preferring the opposite direction. On the population level, this results in an increased selectivity for the attended feature (Martínez-Trujillo and Treue, 2004).

In summary, attention strongly modulates neuronal activity at all levels of cortical visual processing. Both spatial and feature-based attention exert prominent influences on processing of visual motion in area MT. The following work demonstrates, for the first time, a neuronal correlate of object-based attention for individual MT neurons. Furthermore, this work includes several behavioral experiments examining influences of the different types of attention on processing of visual motion. 


\section{Chapter 2}

\section{Original articles and manuscripts}

This chapter contains the following articles and manuscripts:

- Katzner, S., Busse, L. \& Treue, S. Object-based attention modulates activity of single neurons in primate visual cortex. Prepared for submission.

- Katzner, S., Busse, L. \& Treue, S. (2006). Feature-based attentional integration of color and visual motion. Journal of Vision, 6(3), 269-284.

- Katzner, S., Busse, L. \& Treue, S. Anticipation of impending signals lowers decision criterion without affecting perceptual sensitivity. Prepared for submission.

- Busse, L. \& Katzner, S. (2006). The time course of shifting visual attention. Journal of Neuroscience, 26(15), 3885-3886.

- Busse, L., Katzner, S. \& Treue, S. (2006). Spatial and feature-based effects of exogenous cueing on visual motion processing. Vision Research, 46(13), 2019-2027. 


\subsection{Object-based attention modulates activity of single neu- rons in primate visual cortex}

Recent single-unit studies in awake behaving primates have revealed the neural correlates of spatial and feature-based attention in various cortical visual areas. First, when observers direct attention to a certain location in the visual field (spatial attention), neurons whose receptive fields overlaps with the attended region respond more strongly compared to when attention is directed somewhere else. Second, when attention is directed to non-spatial stimulus features, like a color or direction of motion (feature-based attention), neuronal activity is enhanced if the attended feature matches the preferences of the neurons under study, independent of the spatial focus of attention.

While recent psychophysical and fMRI studies have also shown that attention can be directed to objects, there is yet no single-unit evidence for the core prediction of theories of object-based attention: attending to a single feature of an objects results in an enhanced processing of all other features belonging to the same object, even if they are irrelevant.

In the current study, we have directly tested this prediction by recording extracellular activity from individual direction-selective neurons in macaque area MT, while two monkeys were attending to the color of a moving stimulus. Our results are consistent with theories of objectbased attention and provide the first single-unit evidence for an object-based transfer of attention between visual features of the an attended object. 


\title{
Object-based Attention Modulates Activity of Single Neurons in Primate Visual Cortex
}

\author{
Steffen Katzner, ${ }^{1,2}$ Laura Busse, ${ }^{1,2}$ Stefan Treue ${ }^{1,2,3 *}$ \\ ${ }^{1}$ Cognitive Neuroscience Laboratory, German Primate Center, Göttingen, Germany \\ ${ }^{2}$ Bernstein Center for Computational Neuroscience, Göttingen, Germany \\ ${ }^{3}$ Department of Biology, University of Göttingen, Germany
}

*To whom correspondence should be addressed; E-mail: treue@ gwdg.de

The behavioral and neurophysiological consequences of directing visual attention to a spatial location or to non-spatial stimulus features are well-known. Additionally, psychophysical and imaging studies show that attending to one feature of an object, such as its color, leads to a transfer of attention to all the other features of the same object. Here we document for the first time the neural correlate of such a transfer and its consequences for the processing of unattended stimuli in single-unit recordings from area MT in primate visual cortex.

Visual scenes typically contain much more information than our brains can fully process. To cope with this deluge, we can direct attention to a spatial location in the visual field (1), to non-spatial stimulus features, like a specific color or motion direction (2), or even to whole objects (3). Many single-unit studies in awake behaving primates have demonstrated neural correlates of spatial and feature-based attention in various areas of visual cortex (4-7). While 
recent psychophysical (8-12) and functional brain imaging studies $(13,14)$ have shown that the overall organization of visual features into perceptual groups, or 'objects', can also strongly influence the allocation of attention, to date there is no single-unit evidence for the core prediction of theories of object-based attention: attention directed to a single feature of an object will transfer to all other features of the same object, thereby enhancing the processing of even those features that are currently irrelevant. To test this prediction, we recorded extracellular activity from individual neurons in the middle temporal area (MT) of the visual cortex of two macaque monkeys while they were attending either to the direction of motion or to the color of a moving random dot pattern (RDP). Because MT neurons are highly selective for processing of motion direction and speed, rather than color $(15,16)$, MT activity provides a sensitive and reliable measure of motion signal processing under different attentional conditions. Hence, an objectbased attentional transfer between visual features should be reflected in an enhanced processing of the motion signal, even if attention is directed to the color of a moving stimulus.

Our display consisted of two colored RDPs moving either in the preferred or in the null direction of the neuron under study. One RDP was placed inside the classical receptive field (RF) of the neuron, the other one outside at an equal eccentricity in the opposite visual hemifield. In separate blocks of trials, the monkeys attended either to the motion direction or to the color in one of the two RDPs in order to perform a direction or a color task, respectively. In the direction task, the monkeys had to detect a brief change in the direction of motion of the cued stimulus. In contrast, in the color task, the monkeys were required to detect a brief change in the color (Fig. 1). To ensure that the monkeys attended to the cued feature of the cued stimulus, we used different types of distractors, randomized in time and order (fig. S1). For instance, in the direction task, the cued stimulus could also change its color, or the irrelevant stimulus could change its direction of motion or color. To further ensure that the monkeys were accurately following the attentional instructions, we only included those individual blocks of 
trials in all subsequent analyses, for which statistical evaluation confirmed behavior according to the attentional instructions (fig. S2).

Data from a representative MT neuron are shown in Fig. 2A. The dotted traces show the well-known neural correlate of spatial attention (17): Attending to the motion signal of a preferred-direction stimulus inside (dotted red trace) versus outside the RF (dotted black trace) enhances neuronal activity. Remarkably, processing of the motion signal is also enhanced if attention is directed to the color of a preferred-direction stimulus inside (solid red trace) versus outside the RF (solid black trace). This effect is also evident in the population average (Fig. 2B) and is consistent with theories of object-based attention. Figure $2 \mathrm{C}$ shows the distribution of the attentional effect for the 58 individual neurons recorded, separately for the direction (gray bars) and the color task (blue bars). In the direction task, attention enhances processing of the motion signal by $18.8 \% \pm 6.8 \%(18)\left(p=2.17^{-6}\right.$, one-sample $t$ test). Most important, attention to the color of a moving stimulus enhances processing of the irrelevant motion signal by $18.3 \% \pm$ $6.6 \%$ ( $p=2.21^{-6}$, one-sample $t$ test). This effect is statistically reliable for individual monkeys ( $p=0.02$ with $n=15$ cells for monkey A, and $p=0.00002$ with $n=43$ cells for monkey B). The size of the attentional effects in the direction and color task are positively correlated across single neurons (fig. S3A), ruling out the possibility that some neurons show an attentional effect only in the direction, and others only in the color task. Furthermore, if attention is directed to the stimulus inside the RF, average activity in the color task (Fig. 2B, solid red trace) is not different from average activity in the direction task (Fig. 2B, dotted red trace) $(p=0.89$, paired t-test). In fact, single-unit activity in these two conditions is perfectly correlated $(r=0.99$, $p=2.2^{-16}$ ). These results show that attention to the color of a moving stimulus transfers to its direction of motion, resulting in an enhanced processing of the irrelevant motion signal. This is consistent with a recent report, where multi-unit activity in primary visual cortex was enhanced if distant segments of a relevant object overlapped with the RFs of the recording site (19). 
Where could such a transfer take place? Recent behavioral (20) and functional magnetic resonance imaging (fMRI) (21) studies have reported a global attentional modulation of responses to irrelevant stimulus features, even outside the spatial focus of attention. According to the proposed mechanism, attending to a particular color will first result in a global enhancement of processing of this color (feature-based attention). Second, spatio-temporally co-localized color and motion features will be bound together automatically throughout the visual field, leading to an enhanced processing of the irrelevant motion signal in those stimuli having the attended color. Our experimental paradigm allows us to test for such an enhanced processing of the irrelevant feature outside the spatial focus of attention by comparing neuronal activity between conditions in which the monkeys' attention was directed to the motion or the color of a preferred- versus null-direction stimulus outside the RF, while the stimulus inside always moved in the preferred direction of the recorded neuron. Activity for a single MT neuron is shown in Fig. 3A. The dotted lines confirm the effect of feature-based attention in the direction task $(22,23)$, i.e., attending to the preferred (dotted red trace) versus null direction (dotted black trace) outside the RF increased responses to the irrelevant stimulus in the RF. Of central importance, this increase in firing rates was also observed in the color task (solid lines): firing rates were increased if attention was directed to the color of a preferred- (solid red trace) versus null-direction stimulus (solid black trace) outside the RF. Fig. 3B documents the same effects when pooling across our sample of 58 neurons. The distribution of attentional modulation for all neurons studied is shown in Fig. 3C. Attending to the preferred versus null direction outside the RF increases responses by $11.6 \% \pm 5.7 \%(18)$ in the direction task (gray bars) $(p=0.0002$, one-sample $t$ test). When attention is directed to the color of a preferred- versus null-direction stimulus outside the RF, activity is enhanced by $9.2 \% \pm 5.5 \%$ (blue bars, $p=0.0015$, onesample $t$ test). Again, this effect is also statistically significant for the monkeys individually ( $p=0.04$ with $n=15$ in monkey A, and $p=0.008$ with $n=43$ for monkey B). There is no 
difference in the strength of the attentional effect between the color and direction task ( $p=0.45$, paired $t$ test), and the size of the attentional effects across individual neurons in these two tasks is positively correlated (S2B). Thus, the results in the color task demonstrate enhanced processing of irrelevant motion signals outside the spatial focus of attention. However, our data are inconsistent with an attentional enhancement that results from an automatic binding of features throughout the visual field. According to such a mechanism, enhanced processing of the irrelevant motion signal outside the spatial focus of attention should only be observed if the color of the irrelevant stimulus matches the attended color. Fig. 4A shows that there is no difference in firing rates between identically colored (red trace) and differently colored (black trace) stimulus pairs ( $p=0.23$, paired t-test), with the distribution of the corresponding attentional index being centered on zero (Fig. 4B). Consequently, the pattern of our results is best accounted for by an object-based transfer of attention from color to direction of motion, taking place at the spatial focus of attention, which is then followed by a global activation of the corresponding motionfeature system. Thus, firing rates of MT neurons can be modulated by attention if one of the irrelevant features of an attended object outside the RF matches the preferences of the neuron under study. Such a mechanims could also account for a recent psychopysical demonstration of an attentional transfer not only between features of an attended object, but also to remote locations in the visual field (24).

While it is generally assumed that color and direction of motion are processed in anatomically distinct and functionally separate visual processing streams, several neurophysiological studies have shown that information about color is indeed available to the visual motion processing system (25-30). Given such evidence, we want to emphasize that our findings do not reflect a feature-based effect of color. First, we established neuronal isoluminance by presenting pairs of yellow and blue RDPs that provided equally strong input for individual MT cells (31). Second, even if MT neurons showed a preference for one over the other color, any 
feature-based of color effect would cancel out since we averaged across the two colors. Third, we also separately analyzed those trial pairs where the color of the attended preferred- and the attended null-direction stimulus outside the RF was the same. We found a strong trend for the same object-based transfer of attention, but the reduced number of trial pairs in the analysis prevented the effect from reaching statistical significance $(p=0.09)$. Taken together, we can reject the possibility that our results reflect a feature-based effect of color.

In summary, our data show a correlate of object-based attention at the single-neuron level in primate visual cortex. In macaque area MT, processing of an irrelevant motion signal was enhanced if attention was directed to the color of a moving stimulus, that matched the neurons preferences for direction of motion. Beyond a simple object-based effect, we demonstrate that, even outside the spatial focus of attention, irrelevant motion signals receive enhanced attentional processing. Therefore, we propose a mechanism consisting of an object-based attentional transfer at the spatial focus of attention, followed by a global activation of the motion-feature system. Assuming that the transfer applies to all stimulus features, this object-based attentional mechanism would enhance the saliency of all those objects in a visual scene that share at least one feature with the currently attended object.

\section{References and Notes}

1. M. I. Posner, Quarterly Journal of Experimental Psychology 32, 3 (1980).

2. M. Sàenz, G. T. Buraĉas, G. M. Boynton, Nature Neuroscience 5, 631 (2002).

3. J. Duncan, Journal of Experimental Psychology: General 113, 501 (1984).

4. R. Desimone, J. Duncan, Annual Review of Neuroscience 18, 193 (1995).

5. S. Treue, Trends in Neurosciences 24, 295 (2001). 
6. J. H. Reynolds, L. Chelazzi, Annual Review of Neuroscience 27, 611 (2004).

7. J. H. R. Maunsell, S. Treue, Trends in Neurosciences In Press (2006).

8. J. H. Reynolds, S. Alborzian, G. R. Stoner, Vision Research 43, 59 (2003).

9. J. F. Mitchell, G. R. Stoner, M. Fallah, J. H. Reynolds, Vision Research 43, 1323 (2003).

10. J. F. Mitchell, G. R. Stoner, J. H. Reynolds, Nature 429, 410 (2004).

11. E. Blaser, Z. W. Pylyshyn, A. O. Holcombe, Nature 408, 196 (2000).

12. M. Valdés-Sosa, A. Cobo, T. Pinilla, Cognition 66, B13 (1998).

13. K. M. O'Craven, P. E. Downing, N. Kanwisher, Nature 401, 584 (1999).

14. M. A. Schoenfeld, et al., Proceedings of the National Academy of Sciences 100, 11806 (2003).

15. D. J. Felleman, D. C. Van Essen, Cerebral Cortex 1, 1 (1991).

16. L. G. Ungerleider, M. Mishkin, Analysis of Visual Behavior, D. J. Ingle, M. A. Goodale, R. J. W. Mansfield, eds. (MIT Press, Cambridge, MA, 1982), pp. 549-586.

17. S. Treue, J. H. R. Maunsell, Nature 382, 539 (1996).

18. $95 \%$-confidence interval.

19. P. R. Roelfsema, V. A. Lamme, H. Spekreijse, Nature 395, 376 (1998).

20. D. Melcher, T. V. Papathomas, Z. Vidnyanszky, Neuron 46, 723 (2005).

21. W. Sohn, S. C. Chong, T. V. Papathomas, Z. Vidnyanszky, Neuroreport 16, 1389 (2005).

22. S. Treue, J. C. Martínez-Trujillo, Nature 399, 575 (1999). 
23. J. C. Martínez-Trujillo, S. Treue, Current Biology 14, 744 (2004).

24. G. M. Boynton, V. M. Ciaramitaro, A. C. Arman, Vision Research In Press, (2006).

25. K. R. Gegenfurtner, et al., Visual Neuroscience 11, 455 (1994).

26. K. R. Dobkins, T. D. Albright, Journal of Neuroscience 14, 4854 (1994).

27. L. J. Croner, T. D. Albright, Journal of Neuroscience 19, 3935 (1999).

28. A. Thiele, K. R. Dobkins, T. D. Albright, Neuron 32, 351 (2001).

29. E. Seidemann, A. B. Poirson, B. A. Wandell, W. T. Newsome, Neuron 24, 911 (1999).

30. J. J. Nassi, D. C. Lyon, E. M. Callaway, Neuron 50, 319 (2006).

31. Materials and methods are available as supporting material on Science Online.

32. This research is supported by the BMBF grant 01GQ0433. 


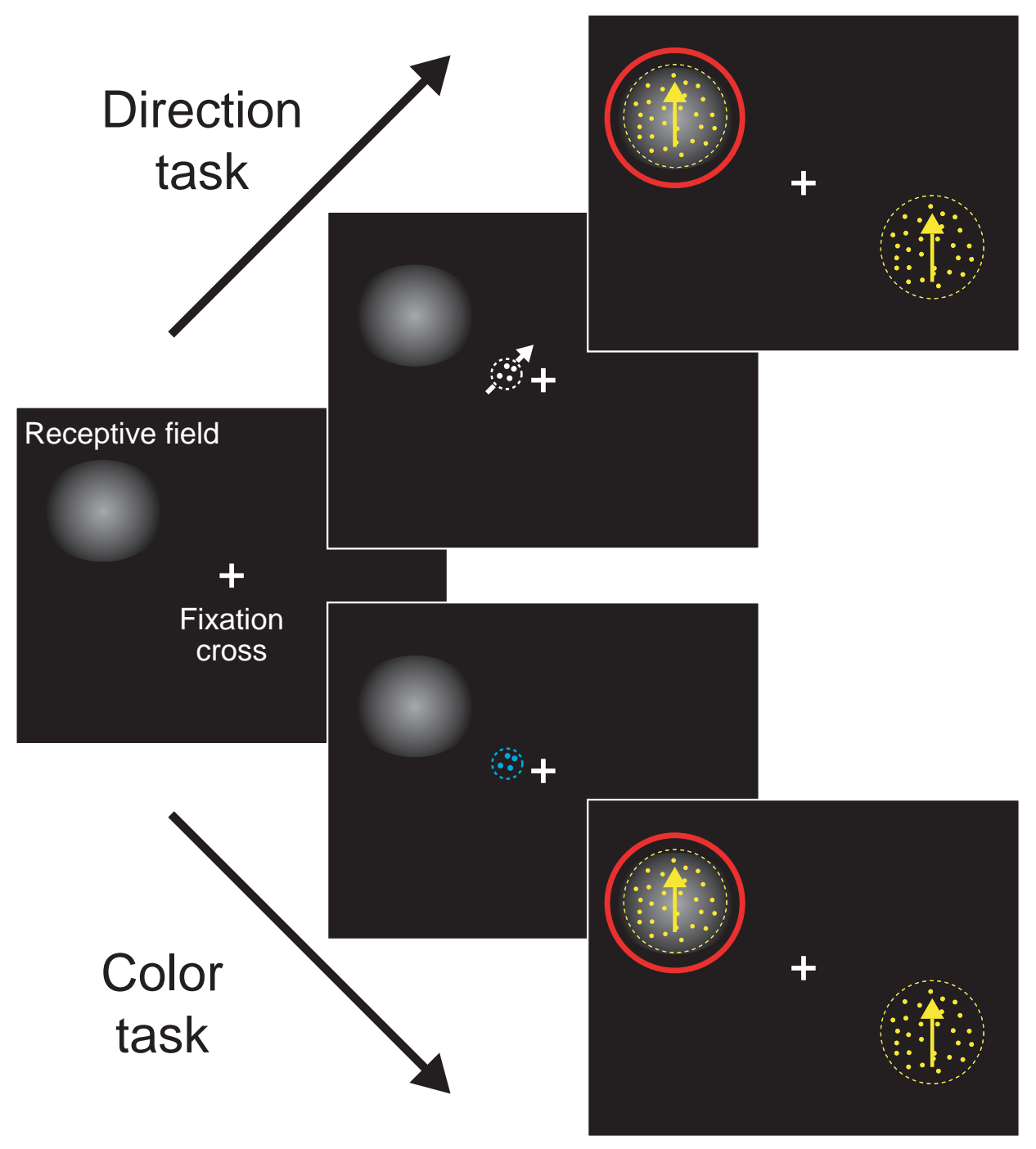

Figure 1: Selective attention task. Trial sequence for the direction task (upper sequence) and color task (lower sequence). After the monkeys fixated and depressed a lever, a small cue briefly appeared, indicating the spatial location of the upcoming target stimulus and the attentional task to be performed. In the direction task, the cue was a small, achromatic moving RDP and instructed the monkey to respond to a change in direction of the cued stimulus. In the color task, the cue was a stationary, colored RDP, instructing the monkey to respond to a change in the color of the cued stimulus. After cue offset, two RDPs were presented at equal eccentricity in opposite visual hemifields, one of them inside the RF of the neuron under study. The red circle indicates the focus of attention. 

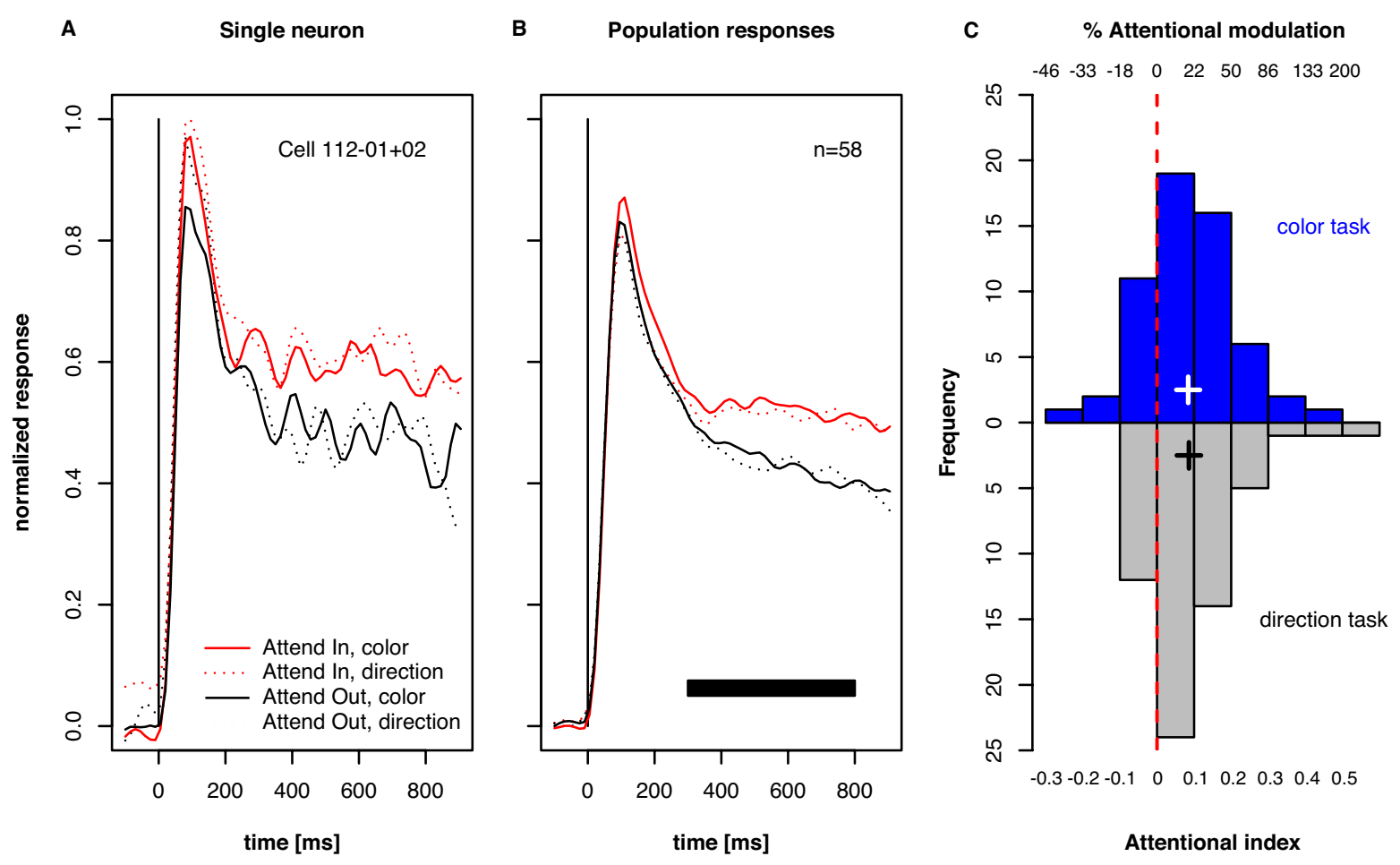

Figure 2: Effects of directing attention to different features of a colored, moving stimulus. (A) Attending to the motion signal of a preferred-direction stimulus inside (red dotted trace) versus outside (black dotted trace) the RF enhances activity of a representative MT neuron. Remarkably, the same modulation is evident if attention is directed to the color of these stimuli (solid traces). (B) Firing rates averaged over a population of 58 MT neurons. (C) Distribution of the attentional effects for the recorded population, separately for conditions in which attention was directed to the motion signal (gray bars) or to the color (blue bars). Binning is based on the attentional index $A I=\left(f r_{\text {in }}-f r_{\text {out }}\right) /\left(f r_{\text {in }}+f r_{\text {out }}\right)$, where $f r$ represents the firing rate in the corresponding attentional condition for the time window marked by the black horizontal bar. The top scale gives the corresponding percentages. Both histograms are shifted to positive values, indicating that attention modulates processing of the motion signal in MT, even if directed to the color of a moving stimulus (the cross indicates the mean attentional index, i.e. the geometric mean of the attentional modulation, horizontal arms span the 95\%-confidence interval). 

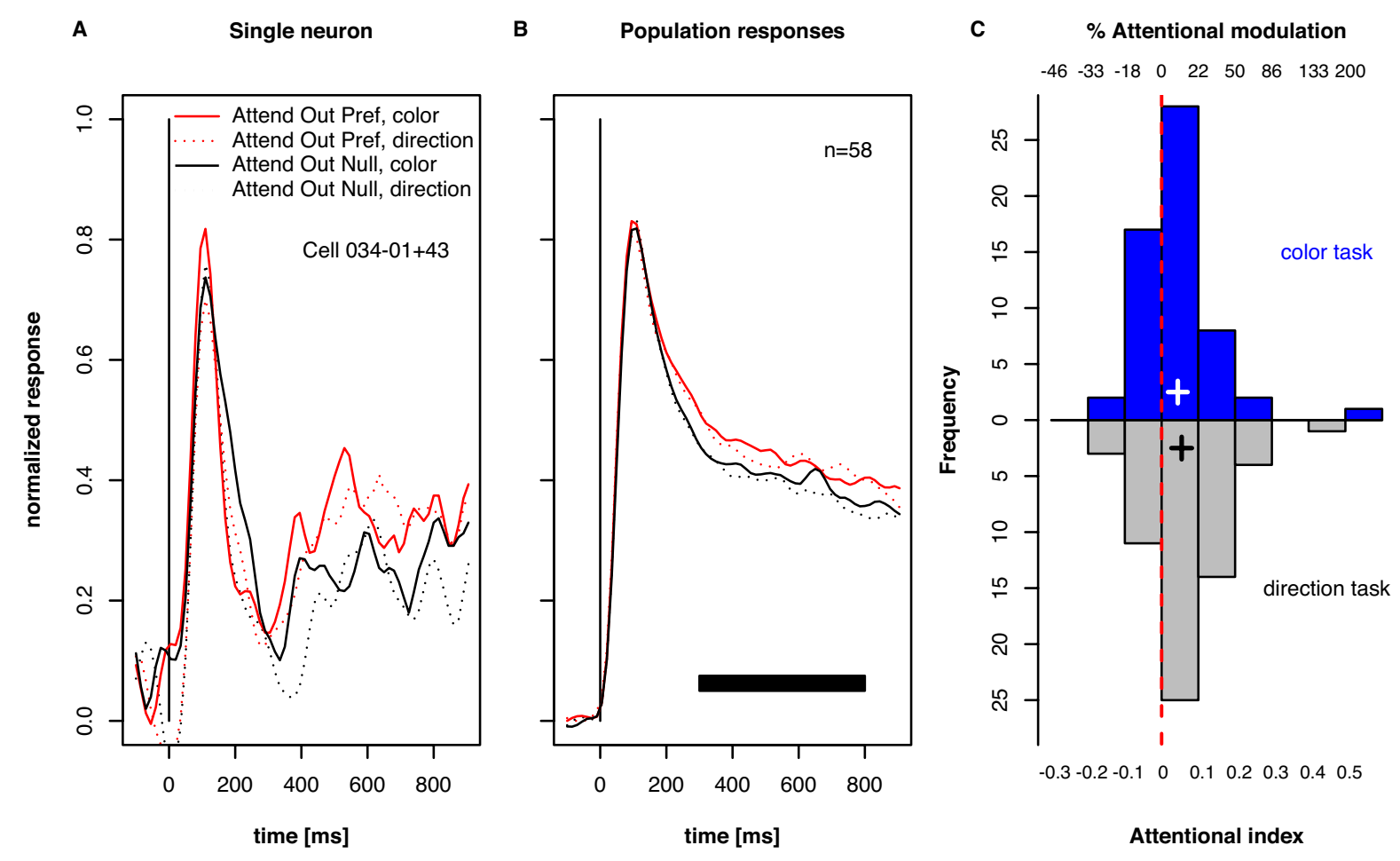

Figure 3: Effects of directing attention to different features of a colored motion stimulus outside the RF. (A) Attending to the motion signal of a preferred-direction (red dotted trace) versus null-direction stimulus (black dotted trace) outside the RF enhances activity of a representative MT neuron. Most important, the same modulation is evident if attention is directed to the color of these stimuli (solid traces). (B) Firing rates averaged over a population of 58 MT neurons. (C) Distribution of the strength of attentional modulation for the recorded population, separately for conditions in which attention was directed to the motion signal (gray bars) or to the color (blue bars). Binning is based on the attentional index $A I=\left(f r_{\text {pref }}-f r_{\text {null }}\right) /\left(f r_{\text {pref }}+f r_{\text {null }}\right)$, where $f r$ represents the firing rate in the corresponding attentional condition for the time window marked by the black horizontal bar. The top scale gives the corresponding percentages. Both histograms are shifted to positive values, indicating that attention to color modulates processing of the irrelevant motion signal in MT, even outside the spatial focus of attention (the cross indicates the mean attentional index, horizontal arms span the 95\%-confidence interval). 

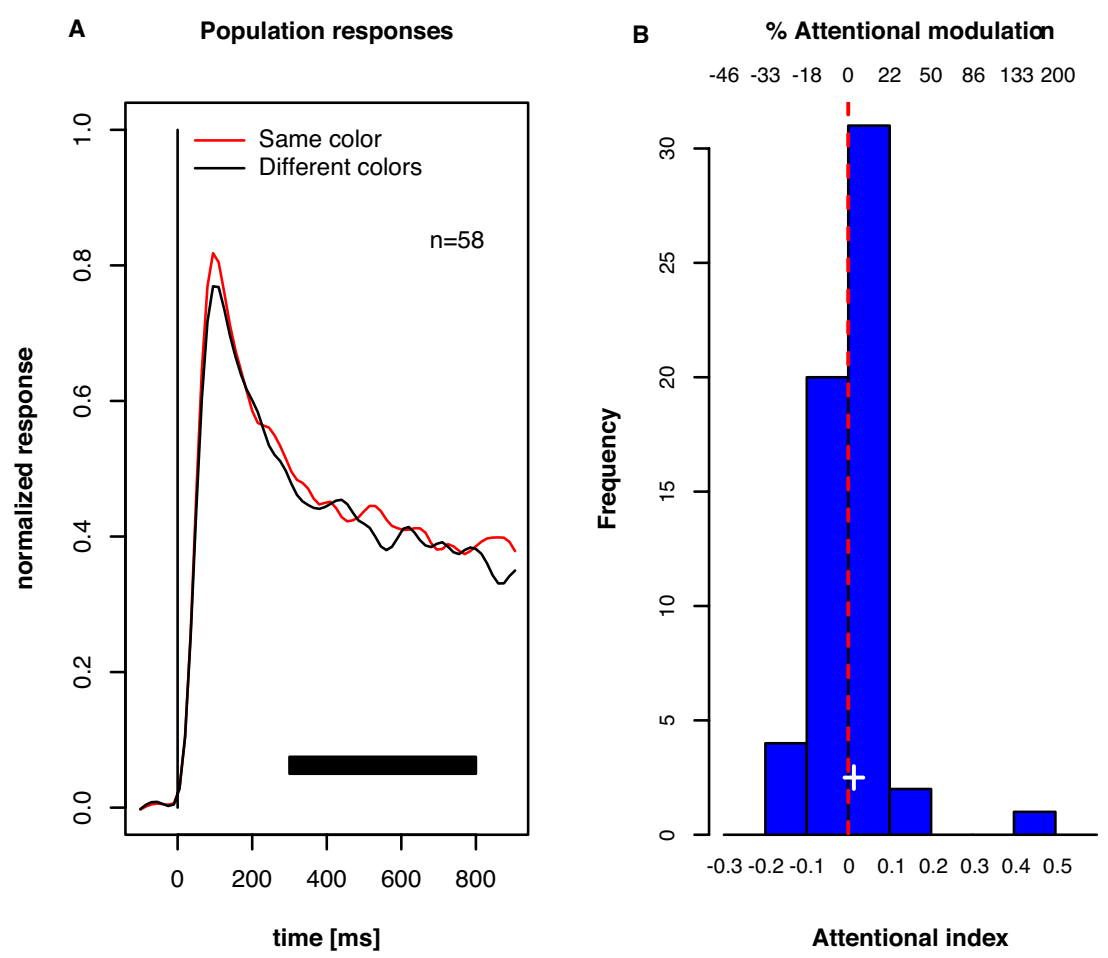

Figure 4: The object-based transfer of attention cannot be explained by mechanisms of perceptual grouping. (A) Average firing rates of $58 \mathrm{MT}$ neurons with attention directed to the color of the stimulus outside the RF. Here, both stimuli always move in the preferred direction of the recorded neuron, but have the same versus different colors. If global enhancement of processing of the irrelevant motion signal were mediated by a perceptual grouping mechanism, firing rates should be higher if the attended color matches (red trace) the color of the stimulus inside the RF, compared to when these colors are different (black trace). Inconsistent with this mechanism, average activity in the time window from 300-800 ms (black horizontal bar) showed no difference ( $p=0.23$, paired t-test). (B) Distribution of attentional indices $A I=\left(f r_{\text {same }}-f r_{\text {diff }}\right) /\left(f r_{\text {same }}+f r_{\text {diff }}\right)$ for the indicated time window, which is centered on zero. 


\title{
SUPPORTING ONLINE MATERIAL
}

\section{Object-based Attention Modulates Activity of Single Neurons in Primate Visual Cortex}

\author{
Steffen Katzner, Laura Busse, Stefan Treue
}

\section{Materials and Methods}

All experimental procedures were approved by the local animal research committee and complied with relevant laws and institutional guidelines. Headpost and recording chamber were implanted using standard surgical techniques (23).

Behavioral task. Two monkeys (Macaca mulatta) were trained to attend either to the motion signal (direction task) or to the color (color task) of a moving random dot pattern (RDP) (Fig. 1). To start a trial, the animal had to maintain fixation within a window of $1.25^{\circ}$ radius, centered on a $0.2^{\circ}$ fixation square. $150 \mathrm{~ms}$ after depressing a lever, a cue appeared for $500 \mathrm{~ms}$, signaling the position of the relevant stimulus (target location) and indicating whether a direction or a color task was required (target feature). After the cue was removed, two moving colored RDPs were presented at equal eccentricity in opposite visual hemifields, one of them inside the classical receptive field $(\mathrm{RF})$ of the neuron under study. To ensure that the monkeys were correctly attending to the cued feature of the cued stimulus, one or two of the following events could happen within the next 500-3550 ms, randomized in time and order: the target feature appeared at the target location, the target feature appeared at the uncued location ('wrong location' distractor), the uncued feature appeared at the target location ('wrong feature' distractor), or the uncued feature appeared at the uncued location ('wrong location and feature' distractor) (fig. S1). In the direction task, the monkeys received a liquid reward for releasing the lever within 
a response time window of $100-500 \mathrm{~ms}$ following a brief change in the direction of motion of the cued stimulus. In contrast, in the color task the monkeys were rewarded for responding to a brief change in the color of the cued stimulus. Trials were ended immediately following any response. If only distractor events occurred, the monkeys were required to continue depressing the lever and were rewarded at the end of the trial (4050 ms after cue offset). While distractor events at the wrong location allowed us to control that the monkeys attended to the target location, distractor events in the wrong feature guaranteed that they selectively attended to the target feature. Trials were terminated without reward if the monkeys responded to any of the distractor types, did not respond to the target feature at the target location, or broke fixation. Color and direction tasks were performed in separate blocks of trials, alternating with every 20 correctly completed trials.

Apparatus and visual stimuli. Monkeys were seated in a primate chair with their head restrained at a distance of $57 \mathrm{~cm}$ from a computer monitor (resolution 40 pixels per degree of visual angle, refresh rate $76 \mathrm{~Hz}$ ). The eye position was monitored with a high-speed videobased eye tracker at a sampling rate of $230 \mathrm{~Hz}$ (ET49, Thomas Recording, Giessen, Germany). Our stimuli consisted of RDPs moving within a stationary virtual aperture. A single dot subtended $0.1^{\circ}$ of visual angle and the dot density was 8 dots $/ \mathrm{deg}^{2}$. The size of the RDP, the speed of the dots, and the direction of motion were selected to match the preferences of the neuron under study. On a given trial, each RDP was independently assigned one of two neuronally isoluminant colors (blue versus yellow), and one of two motion directions (preferred versus null direction of the recorded neuron). In case of a direction change, all dots simultaneously changed their direction by $30-60^{\circ}$, depending on eccentricity and speed of the target stimulus. For color changes, $80 \%$ of all dots changed their color to either yellow or blue, depending on the original stimulus color. All changes lasted for $132 \mathrm{~ms}$ before the original stimulus properties were restored. The cues consisted of small RDPs subtending $0.75^{\circ}$ of visual angle, with a dot size 
of $0.075^{\circ}$ and a density of $40 \mathrm{dots} / \mathrm{deg}^{2}$. They were always presented at a distance of $2^{\circ}$ from fixation, positioned on a virtual line connecting the fixation point to the target location. For the direction cue, dots were achromatic and moved at a speed of $3^{\circ} / \mathrm{s}$ in the direction which had to be detected. In the color task, the dots were stationary and were plotted in the to-be-detected color.

Neuronal recording and data collection. Single-unit activity was recorded from area MT using a five-channel multi-electrode recording system (Mini-matrix, Thomas Recording, Plexon data acquisition system). For most of the recording sessions, five electrodes were simultaneously advanced to isolate individual MT neurons with overlapping RFs. Cells were characterized as MT neurons based on directional tuning, receptive field location, and position in the cortex. The locations and sizes of individual RFs were mapped manually using a moving bar. Directional and speed tuning were determined by presenting a single RDP inside the joint RF, moving in 12 different directions for each of 8 different speeds $(0.5-64 \mathrm{deg} / \mathrm{s})$, while monkeys were maintaining fixation. Preferred direction was estimated by fitting a Gaussian to the individual neuronal responses, plotted as a function of stimulus direction, separately for each speed. Out of the 58 recorded neurons, 23 were obtained from parallel recordings (either from two, three, or four cells simultaneously). For these groups of cells, the stimulus for the subsequent experiment was optimized for the neuron exhibiting the strongest direction selectivity. Neuronal isoluminance was established by presenting a preferred-direction RDP inside the receptive field (3.7-5.7 s) that changed its color every $500 \mathrm{~ms}$, while the monkey held fixation. Colors were randomly selecting from a sample consisting of a single blue of fixed intensity $\left(45 \mathrm{~cd} / \mathrm{m}^{2}\right)$ and various intensities of yellow, bracketing the level of intensity for blue (31-63 $\mathrm{cd} / \mathrm{m}^{2}$ ). By selecting the appropriate level of intensity for yellow we made sure that both colors provided equally strong inputs for individual MT neurons. 
Analysis of behavioral data. The comparison between neuronal activity between the direction and color task critically requires that the monkeys were reliably following the attentional instructions given by the cue. To ensure this, we examined the monkeys' behavioral performance in every single block of trials for each recording session. Specifically, we calculated the probability that the number of hits within a block could be obtained by chance, given the total number of trials in that block, and assuming that the monkey was guessing as to whether he should be responding to a color or a direction change (binomial test with probability $p$ of success $=0.5$ ) (fig. S2A). For all further analyses, we included only those individual blocks of trials, for which the behavioral performance was reliably different from chance level $(p<0.05)$. Subsequently, an Analysis of variance (ANOVA) was performed, separately for targets and distractors, to test for systematic dependencies of performance on the two attentional tasks (fig. S2B). Target-related performance was assessed with a one-way ANOVA involving the withinsubjects factor type of task (direction task vs. color task). Both monkeys successfully detected most of the targets, with a slightly better performance in the color task $(91.08 \%)$ than in the direction task (88.61\%). Distractor-related performance was examined with a two-way ANOVA involving the within-subjects factors type of task and type of distractor ('wrong location' versus 'wrong dimension' versus 'wrong location \& dimension'). On average, both monkeys successfully ignored most of the distractors $(89.32 \%)$, with better performance for location distractors (96.89\%) than for dimension \& location distractors $(89.54 \%)$, and for dimension distractors $(81.53 \%)$. Most important, performance differences between the different distractor types were identical for the color and direction task ( $p=0.1$, Greenhouse-Geyser corrected).

Analysis of neuronal data. For all cells included in the analysis of neuronal data, responses to the preferred direction were at least three times as large as responses to the null direction. For any given recording session, only those blocks of trials were included for which the analysis of behavior ensured that the monkeys were following the attentional instructions (fig. S2). Finally, 
individual trials were only included if they were correctly completed, and for these trials neuronal data were only analyzed until the first change in either of the two stimuli occurred. All firing rates plotted represent values of the spike density function at steps of $15 \mathrm{~ms}$, which were obtained by convolving spike trains with a Gaussian kernel $(\sigma=30 \mathrm{~ms})$. Attentional effects were quantified by computing an attentional index, defined as the difference in firing rates between two conditions, divided by their sum (Fig. 2C, 3C, 4B), after subtraction of spontaneous firing rate. For plotting single neuron data and population activity, neuronal responses were normalized to the peak amplitude evoked by an unattended preferred-direction stimulus presented inside the RF. 

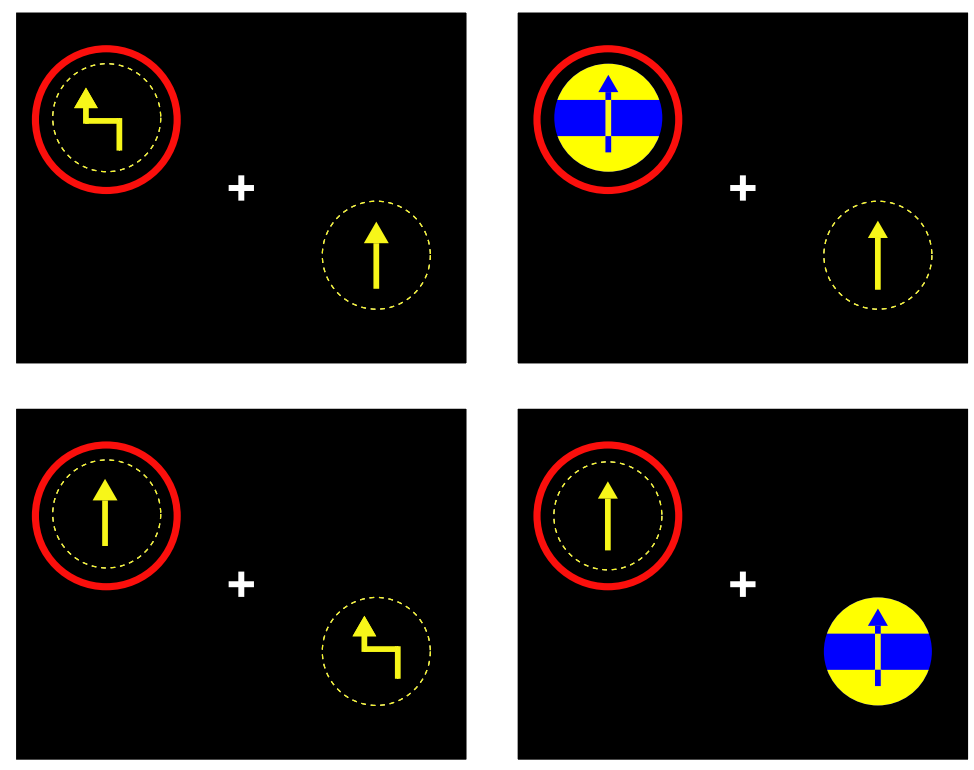

Figure S1: Target event and three different distractor events in the direction task. The monkeys were only rewarded for responding to a change in the direction of motion of the cued stimulus (top left panel). Trials were terminated without reward if they responded to any of the following distractor types: a change in the color of the cued stimulus (top right, 'dimension distractor'), a change in the direction of motion of the uncued stimulus (bottom left, 'location distractor'), or a change in the color of the uncued stimulus (bottom right, 'dimension \& location distractor'). Trials were also terminated without reward if the monkeys missed the target or broke fixation. Corresponding events were used in the color task. 
A

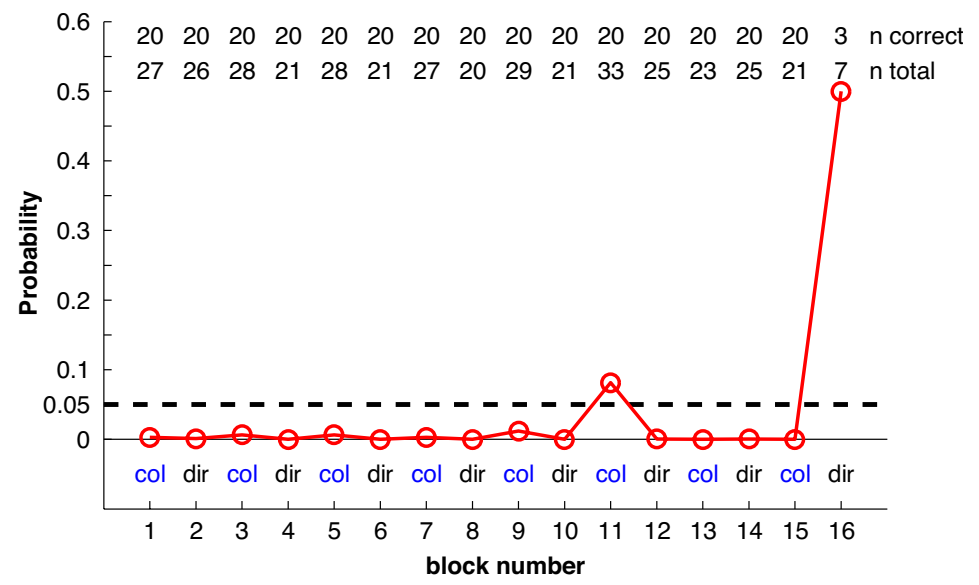

B

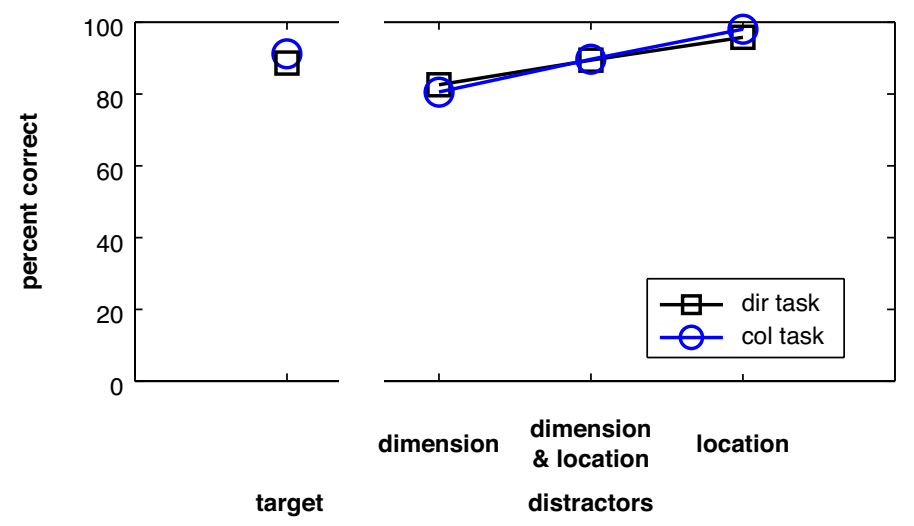

Figure S2: Analyses of behavioral performance. (A) Blockwise analysis of overall behavioral performance. Representative recording session during which the monkey performed 16 blocks of color (col) and direction (dir) tasks, alternating every 20 correctly completed trials. Rows at the top indicate the number of correct trials and the total number of trials (excluding trials that were terminated because of fixation breaks), separately for every block. Depicted is, for every single block, the probability that 20 hits can be obtained purely by chance, given the total number of trials in that block, and assuming that the monkey was guessing (binomial test with probability $p$ of success $=0.5$ ). The dashed line marks the performance-based criterion for including individual blocks in all subsequent analyses. In this particular session, blocks 11 and 16 were excluded (in the last block the monkey only performed 7 trials, after which the recording session was ended). For the remaining 14 blocks, it is obvious that the monkey reliably performed alternating color and direction tasks. (B) Average performance for targets and for the different distractor types. Included are only blocks that meet the behavioral performance criterion. Performance for the targets (left part) was very high and the different distractor types (right part) had, on average, very little impact on performance. The pattern of results validates that the monkeys followed the attentional instructions and selectively attended to the cued feature of the cued stimulus. 
A

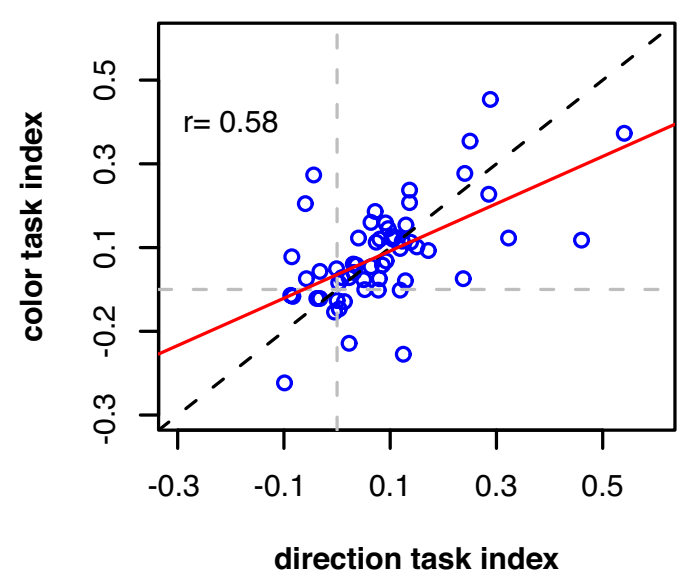

B Attend pref out vs. null out

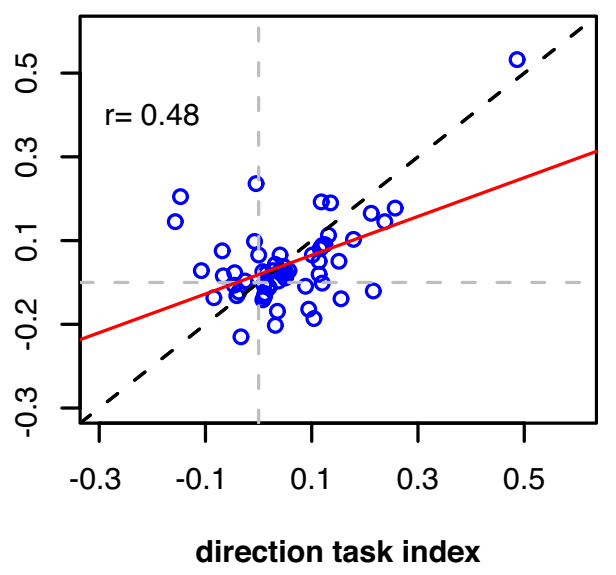

Figure S3: Attentional effects in the direction task and in the color task are positively correlated. The scatter plots shows attentional indices in the direction and the color task, if attention is directed to the preferred-direction stimulus inside versus outside the $\mathrm{RF}(\mathbf{A})$, or to a preferredversus null-direction stimulus outside the RF $(B)$. Circles represent single neurons $(n=58)$. The best-fit least-squares lines are shown in red. An Analysis of covariance (ANCOVA) confirmed that the intercept of the fitted line is not different from zero $(p=0.18)$, and that there is no difference in intercepts between A and B $(p=0.42)$. Here, an intercept term different from zero would indicate systematic differences between the attentional indices in the direction task and color task. While the slope parameter significantly deviates from 1 ( 0.46 for $\mathrm{A}, p=0.00016)$, there is also no difference in slopes between the two conditions $(p=0.51)$. The slope is smaller than 1 because some neurons showed strong attentional effects in the direction task, but somewhat weaker effects in the color task. In addition, there were also a few neurons showing suppression by attention in the direction task, but enhancement in the color task. Overall, however, the ANCOVA confirms that there is a strong positive relationship between attentional effects in the direction and in the color task for individual cells, and that this relationship holds true for both attentional comparisons. 


\subsection{Feature-based attentional integration of color and visual motion}

While the previous manuscript has investigated the effects of focused attention, the current study examines influences of divided attention on processing of color and motion. In divided attention paradigms, subjects are typically required to attend to more than one part of the visual scene or to more than one feature of a single stimulus.

This study consists of four behavioral experiments with human observers. The subjects were instructed to make speeded responses whenever a moving RDP changed its direction of motion, its color, or both. We applied a formal race model to the analysis of RT data to demonstrate that color and motion signals are perceptually integrated rather than processed separately and independently in such a task. We have further examined whether the observed integration requires that changes in color and direction of motion occur at the same location or within the same object. We find that perceptual integration of color and motion signals persists even if these signals occur at separate locations or in different objects. The overall pattern of these results can best be accounted for by a feature-based mechanism of attention which enhances processing of relevant stimulus features across the whole visual field. 


\section{Feature-based attentional integration of color and visual motion}

\section{Steffen Katzner}

\section{Laura Busse}

\section{Stefan Treue}

\author{
Cognitive Neuroscience Laboratory, German Primate Center, \\ Göttingen, Germany

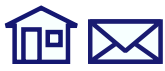 \\ Cognitive Neuroscience Laboratory, German Primate Center, \\ Göttingen, Germany

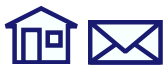 \\ Cognitive Neuroscience Laboratory, German Primate Center, \\ Göttingen, Germany
}

In four variants of a speeded target detection task, we investigated the processing of color and motion signals in the human visual system. Participants were required to attend to both a particular color and direction of motion in moving random dot patterns (RDPs) and to report the appearance of the designated targets. Throughout, reaction times (RTs) to simultaneous presentations of color and direction targets were too fast to be reconciled with models proposing separate and independent processing of such stimulus dimensions. Thus, the data provide behavioral evidence for an integration of color and motion signals. This integration occurred even across superimposed surfaces in a transparent motion stimulus and also across spatial locations, arguing against object- and location-based accounts of attentional selection in such a task. Overall, the pattern of results can be best explained by feature-based mechanisms of visual attention.

Keywords: reaction time, race model, attention, color, motion, transparent motion

\section{Introduction}

Functional specialization is one of the hallmarks of the primate visual cortex. Different attributes of a visual stimulus, like motion, depth, form, and color, are known to be processed in separate areas or even pathways of the visual cortex (Felleman \& Van Essen, 1991). A number of such specialized cortical areas have been identified, and they seem to form at least two processing streams (Ungerleider $\&$ Mishkin, 1982). The ventral pathway (mainly involving areas V1, V2, V4, TEO, and IT) shows specialization for the processing of color and shape. In contrast, areas in the dorsal pathway (V1, V2, V3, MT/MST) analyze information about motion and spatial relations. This notion of spatially separate and functionally independent, parallel processing streams represents an important conceptualization of visual information processing. However, the functional separation is far from complete (Ferrera, Nealey, \& Maunsell, 1992), and a large number of anatomical connections between these two pathways have been demonstrated, providing a neural substrate for interactions.

In reaction time $(\mathrm{RT})$ research, the combined processing of separable sensory signals has been studied with the redundant-target paradigm (Miller, 1982, 1986; Mordkoff \& Yantis, 1993). In a typical experimental situation, two different sensory signals are defined as targets and participants are required to make speeded responses if either of the two targets is detected. Of special interest is a condition in which both targets are presented simultaneously (redundant-target trials). This condition is then compared with those in which either of the targets is presented alone (single-target trials). It is typically found that RTs to redundant targets are faster than RTs to single targets, and this finding is commonly referred to as redundancy gain (Giray \& Ulrich, 1993; Iacoboni \& Zaidel, 2003; Krummenacher, Müller, \& Heller, 2001, 2002; Miller, 1982, 1986, 2004; Miller, Ulrich, \& Lamarre, 2001; Miniussi, Girelli, \& Marzi, 1998; Mordkoff, Miller, \& Roch, 1996; Mordkoff \& Yantis, 1991, 1993; Turatto, Mazza, Savazzi, \& Marzi, 2004).

Essentially, two classes of models have been advanced to explain this redundancy gain. The first class consists of race models. They are based on the idea that the two concurrent sensory signals are processed separately and independently and that responses can be initiated as soon as one of the two signals is detected. Sensory information for the two signals is not combined to initiate a response. Responses to redundant targets are particularly fast because they are produced by the faster of the two detection processes. The term race model illustrates the fact that responses are thought to be initiated by the winner of a race between the two separate detection processes. If one assumes that processing time randomly varies from trial to trial, and that the distributions of processing times for the two signals overlap, it follows that, on average, the time needed by the winner will be less than the time needed by either racer. For this reason, race models have also been characterized as producing statistical facilitation 
(Raab, 1962). The second class of models consists of coactivation models (Miller, 1982). In contrast to race models, sensory information for the two signals is combined to reach some decision criterion based on which responses are initiated. Here, responses to redundant targets are particularly fast because such a criterion will be reached faster if two processes combine in satisfying it. The term coactivation reflects the fact that two processes combine in activating a response.

\section{The race-model inequality}

Miller (1982) has provided a formal test to decide between these two classes of models with experimental RT data. In brief, he showed that all race models have to fulfill the following inequality:

$P\left(\mathrm{RT}<t \mid S_{1}\right.$ and $\left.S_{2}\right) \leq P\left(\mathrm{RT}<t \mid S_{1}\right)+P\left(\mathrm{RT}<t \mid S_{2}\right)$,

where $t$ is the time needed to respond to a signal and $S_{1}$ and $S_{2}$ are the two targets. Intuitively, this inequality formalizes an implicit constraint applying to all variants of race models: Responses to redundant targets $\left(S_{1}\right.$ and $S_{2}$, presented together) cannot be faster than the fastest response to either of the single targets ( $S_{1}$ or $S_{2}$, presented alone) of the single targets. Note that the three terms in this inequality represent the cumulative probability density function (CDF) of RT on redundant- and single-target trials, respectively. The CDFs obtained in a speeded detection task just need to be evaluated at the different values of $t$ to decide between race and coactivation models: If the inequality is violated for any of them, all variants of race models can be rejected.

In this study, we apply this logic to the processing of color and motion signals. We consider performance that is consistent with predictions of race models as evidence for separate and independent processing of these two stimulus dimensions. Conversely, performance inconsistent with predictions of race models would argue against separate and independent processing and rather support crossdimensional integration of color and motion signals.

In Experiment 1, we establish that redundancy gains can be observed for the stimulus dimensions color and direction of motion. We further demonstrate that explanations based on race models can safely be rejected, favoring crossdimensional integration of color and motion signals in speeded target detection tasks. In three subsequent experiments, we investigate in more detail whether the observed integration depends on specific stimulus attributes or task demands. Throughout, we find robust redundancy gains, as well as strong evidence for cross-dimensional processing of color and motion signals.

\section{Experiments}

\section{Experiment 1}

The purpose of Experiment 1 was to investigate whether redundancy gains can be observed for targets defined by a change in color or in the direction of motion. We used moving random dot patterns (RDPs) in a go/no-go target detection task, requiring speeded responses if a particular color (single color target), a particular direction of motion (single direction target), or both (redundant target) were presented and no response if the stimulus did not contain any of the designated targets.

\section{Methods}

The stimulus (Figure 1a) was composed of dots moving within a virtual circular aperture with a diameter of $5 \mathrm{deg}$. The dot density was 2 dots $/ \mathrm{deg}^{2}$ of visual angle. Each dot subtended 0.1 deg of visual angle. The RDP was plotted against a black background and centered on a yellow fixation point that was presented in the middle of the screen. Each trial started with an RDP that rotated around the fixation point at an angular speed of $2.9 \mathrm{deg} / \mathrm{s}$; all the dots were gray. In case of a color change, the dots changed to red, green, or blue. The four colors were equiluminant $\left(25 \mathrm{~cd} / \mathrm{m}^{2}\right)$. In case of a direction change, $70 \%$ of all dots started to translate into one of three linear motion directions: either upward, to the left, or to the right. The remaining $30 \%$ of the dots moved in random directions. This was done to prevent participants from solving the task by tracking a single dot. The stimulus was presented on a VGA monitor (Lacie, Electron22 Blue IV) operating at a refresh rate of $85 \mathrm{~Hz}$ and a resolution of 80 pixels/deg of visual angle. Presentation of the stimulus and recording of the responses were controlled by an Apple Power Mac G4 computer.

At the beginning of an experiment, participants were told which color and direction of motion were defined as targets. They were instructed to respond with a keypress on a computer keyboard (" $\mathrm{H}$ ") if the target color, the target
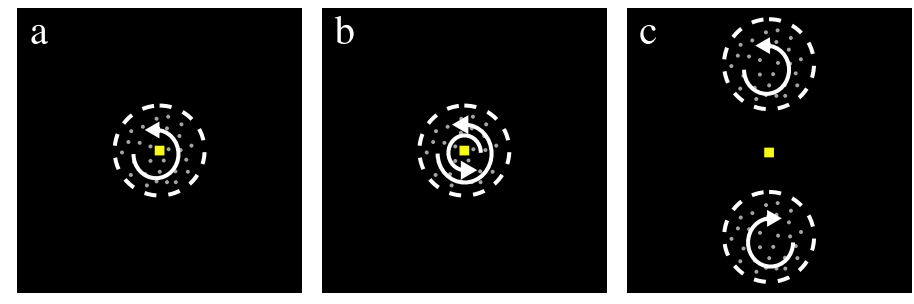

Figure 1. Stimuli used in Experiments 1-4. (a) Experiment 1. A single RDP rotating around the fixation square. (b) Experiment 2. Two superimposed RDPs rotating against each other. (c) Experiments 3 and 4 . Two rotating RDPs centered 3.75 and 7.5 deg above and below fixation in Experiments 3 and 4, respectively. 


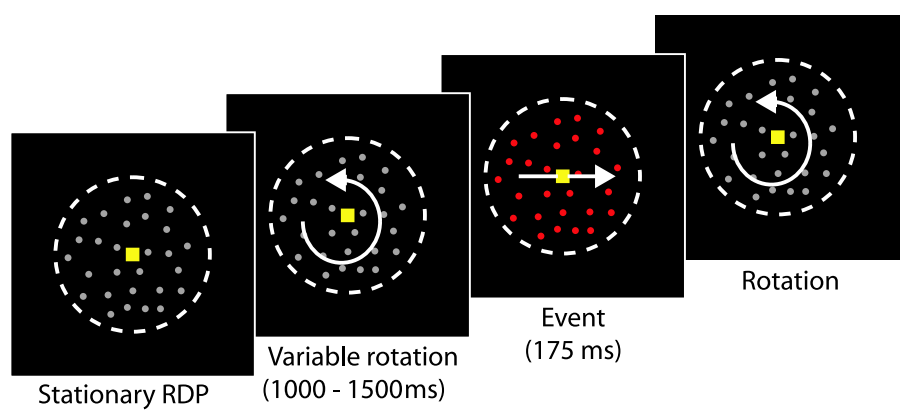

Figure 2. Trial sequence. A stationary pattern of gray dots was present at the beginning of each trial. It then rotated around the fixation square for 1,000-1,500 ms before changing its color, direction of motion, or both. After another period of $175 \mathrm{~ms}$, the RDP changed back to its initial rotation and color.

direction, or both were presented (go trials) and to withhold their response if no target was presented (no-go trials). The participants were told to respond as quickly as possible without making too many errors. The trial sequence is illustrated in Figure 2. A stationary dot pattern was presented, together with the fixation point, until participants triggered the beginning of a trial by pressing the space bar on the keyboard. Following this, the RDP rotated around the fixation point for $1,000-1,500 \mathrm{~ms}$. Then, one of the following events occurred: a change in color, a change in direction of motion, or both. The changes lasted for $175 \mathrm{~ms}$ before the original stimulus properties were restored. Following the participants' response, the stimulus was removed. If no response had been given within 1,000 ms after onset of the event, the trial was terminated and the response was considered a no-go. Auditory feedback was given at the end of each trial. The participants triggered the beginning of the next trial when they were ready to proceed.

Any redundancy gain would result in shorter RTs to redundant targets as compared to single targets. This, however, would be a comparison between a condition with two sensory events (redundant-target trials, with simultaneous changes in direction of motion and color) against a condition with only a single sensory event (single-target trials, with changes either in direction of motion or in color). Because two sensory events represent a much stronger signal compared with a single sensory event, this difference by itself could speed RTs in the redundant-target condition.
To make sure that potential redundancy gains do not result from a difference in the number of sensory events per se, we introduced single-target control conditions having two sensory events. In these control conditions, the single targets were combined with neutral events in the other stimulus dimension (i.e., a single color target was combined with a neutral change in direction, and a single direction target was combined with a neutral change in color). A neutral event is "neutral" in the sense that it appeared equally often in combination with go signals as well as with no-go signals. Consequently, the appearance of a neutral event was uninformative as to whether the participant should respond, whereas the appearance of a no-go event always signaled to withhold the response. Experiment 1 was divided into six blocks of 100 trials each. In a single block, there were 10 signal conditions (5 go and 5 no-go conditions, listed in that order): redundant targets, single color targets, single direction targets (the latter two will be referred to as "single target alone"), single color targets combined with neutral direction changes, single direction targets combined with neutral color changes ("single target + neutral"), redundant no-gos, single color no-gos, single direction no-gos, single color nogos combined with neutral direction changes, and single direction no-gos combined with neutral color changes. Overall, there were $50 \%$ go and $50 \%$ no-go trials. The 10 signal conditions were presented in a pseudorandomized order until 10 correct responses had occurred in each condition. Within each condition, the initial rotation of the RDP was clockwise for five trials and counterclockwise for the remaining five trials. Between blocks, participants were given a break of $5 \mathrm{~min}$. The assignment of three particular colors to go, no-go, and neutral conditions was counterbalanced across participants and remained unchanged throughout the entire experiment. To achieve the same level of difficulty for the go and no-go motion tasks, we always treated upward motion as the neutral direction for all participants. For half of the participants, rightward motion represented the go direction, whereas leftward motion represented the no-go direction; for the other half, this assignment was reversed. Table 1 summarizes one such combination of go, no-go, and neutral events forming the 10 signal conditions.

\section{Participants}

Each participant was tested in two sessions that were performed on separate days. A single session was divided

\begin{tabular}{|c|c|c|c|c|c|c|c|}
\hline & \multirow[b]{2}{*}{ Go signals } & \multirow[b]{2}{*}{ No-go signals } & & \multicolumn{2}{|c|}{ Go signals } & \multicolumn{2}{|c|}{ No-go signals } \\
\hline & & & & Color & Direction & Color & Direction \\
\hline Redundant & Red + right & Green + left & $\begin{array}{l}\text { Single alone } \\
\text { Single }+ \text { neutral }\end{array}$ & $\begin{array}{l}\text { Red } \\
\text { Red + up }\end{array}$ & $\begin{array}{l}\text { Right } \\
\text { Right + blue }\end{array}$ & $\begin{array}{l}\text { Green } \\
\text { Green + up }\end{array}$ & $\begin{array}{l}\text { Left } \\
\text { Left }+ \text { blue }\end{array}$ \\
\hline
\end{tabular}

Table 1. Experiment 1. Combination of go, no-go, and neutral events for participants instructed to detect rightward motion (direction target) or the color red (color target). The color green and leftward motion did not require a response. In the "single + neutral" condition, single color signals were accompanied by neutral direction changes (upward motion), whereas single direction signals were accompanied by neutral color changes (blue). 
into three blocks of approximately $10 \mathrm{~min}$ each. Each participant was instructed to respond to a particular color and a particular direction of motion at the beginning of the experiment. During the first session, participants completed a practice block to become familiar with the task. These practice blocks were not included in the analyses. Twelve participants (four men and eight women; ages 22-36, $M=27.1$, $S D=5.3$ ) were tested in Experiment 1 and were paid for their participation. All had normal or corrected-to-normal vision. They gave informed written consent and were naive as to the purpose of the experiment. All sessions were conducted in a dimly illuminated, quiet testing booth. Participants were comfortably seated with their head resting in a chin and forehead rest at a distance of $114 \mathrm{~cm}$ from the computer monitor.

\section{Data analyses}

Two analyses were performed. The first analysis addressed the redundancy gain and examined whether RTs to redundant targets were faster than RTs to single targets. Mean RTs were determined for each combination of participant and signal condition (redundant target, single color alone, single direction alone, single color + neutral direction, single direction + neutral color). For each participant, we selected the faster of the two mean RTs from the "single target alone" conditions ("fastest single alone"). Analogously, we determined the faster of the two mean RTs in the "single target + neutral" condition ("fastest single + neutral"; see Miller, 1982). Statistical significance was evaluated with a one-way ANOVA involving the withinsubjects factor of target type (redundant target vs. fastest single alone vs. fastest single + neutral).

The second analysis compared RT distributions between conditions to test for violations of the race-model inequality. Following Miller (1982), we compared the sum of the CDFs for the single-target conditions to the CDF for the redundant-target condition. First, we determined the CDFs for each combination of participant, block, and signal condition (redundant target, single color alone, single direction alone, single color + neutral direction, single direction + neutral color) by rank ordering the 10 RTs, which then represent an estimate of the unknown, true $\mathrm{CDF}$ at 10 percentiles (.05-.95). We then computed the sum of the CDFs in the "single target alone" condition ("sum of single alone") and, analogously, the sum of the CDFs in the "single target + neutral" condition ("sum of single + neutral"). The obtained CDFs were then averaged across blocks for a given participant and finally across participants. To test for violations of the race-model inequality, we compared the CDF for the redundant-target condition to the sum of the CDFs for the "single target alone" condition. Analogously, we compared the CDF for the redundant-target condition to the sum of the CDFs for the "single target + neutral" condition. The race-model inequality would be violated if RTs from the redundant-target CDF were faster than corresponding RTs from the summed CDF at any of the percentiles. Statistical significance was evaluated by conducting paired $t$ tests across participants at each of the 10 percentile pairs (redundant vs. "sum of single").

\section{Results and discussion}

\section{Redundancy gain}

Across participants, performance reached $89 \%$ correct for the no-go trials and varied between $99 \%$ and $100 \%$ correct for the five go conditions. Mean RTs across participants are shown in Figure 3. The ANOVA revealed a highly significant difference between mean RTs, $F(2,22)=33.73$, $M S E=126.39, p<.001$, Greenhouse-Geyser corrected. Post hoc analyses confirmed that RTs for redundant targets (328 ms) were faster than RTs for both "fastest single alone" (358 ms, $p<.001)$ and "fastest single + neutral" (363 ms, $p<.001)$. However, RTs for "fastest single alone" were not different from RTs for "fastest single + neutral" $(p=.28)$. A 95\% confidence interval for the main effect of target type indicates that redundancy gains are in the range of 26-40 ms under the present conditions.

\section{Race-model inequality}

Mean CDFs across participants are shown in Figure 4. Comparing the redundant-target $\mathrm{CDF}$ (pentagrams) to the sum of single alone CDF (circles) reveals a clear violation of the race-model inequality because the redundant-target CDF lies above and to the left of the "sum of single alone" CDF (upper panel in Figure 4).

Paired $t$ tests across participants at each of the 10 percentile points confirmed that RTs from the redundant signal CDF were reliably faster than RTs from the "sum of

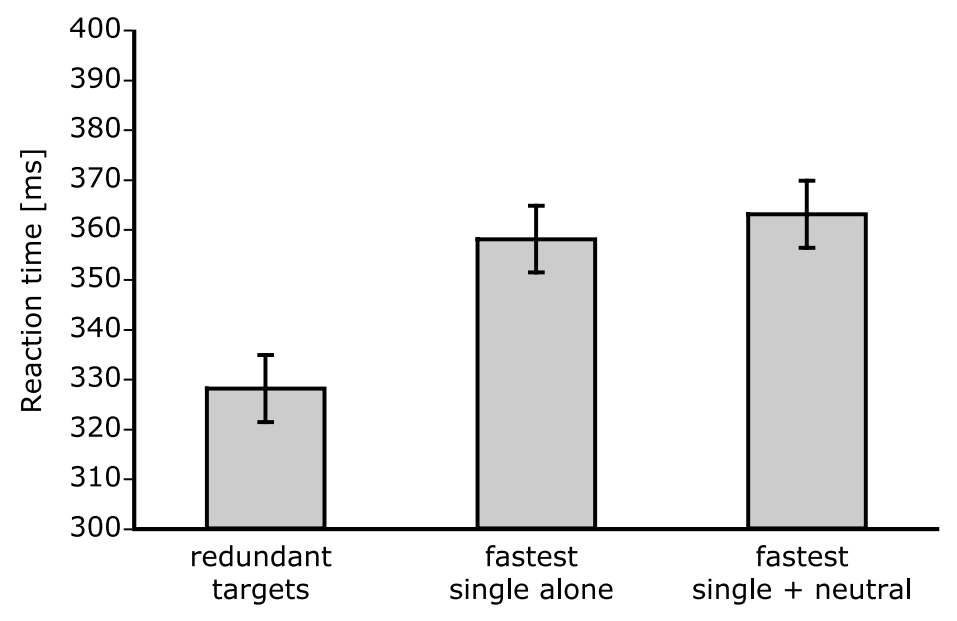

Figure 3. Experiment 1. RTs as a function of target type (redundant target vs. fastest single alone vs. fastest single + neutral). RTs to redundant targets are lower than RTs in the other two conditions ( $p<.001$ for both comparisons). There is no statistical difference between "fastest single alone" and "fastest single + neutral." Error bars indicate the 95\% confidence interval for the main effect of target type (see Loftus \& Masson, 1994). 

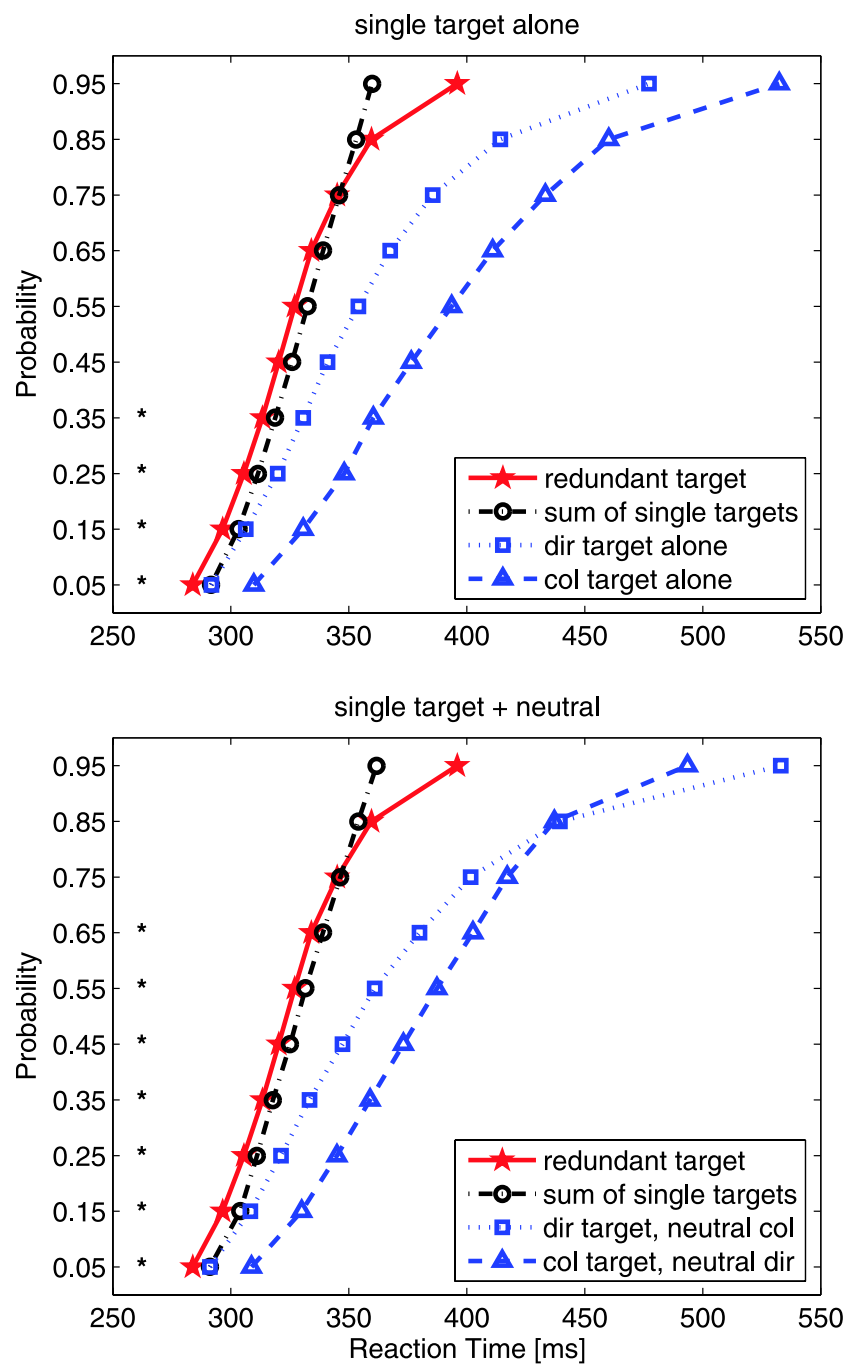

Figure 4. Experiment 1. Violations of the race-model inequality are revealed by comparing the redundant-target CDF (pentagrams) with the sum of the single-target CDFs (circles). Inconsistent with predictions of all race models, the redundant-target CDF lies above and to the left of the CDF for the sum of the single targets. This is the case for both "sum of single alone" (upper panel) and "sum of single + neutral" (lower panel). It shows that the fastest response to redundant targets can be faster than the fastest responses to single targets. Significance markers along the $Y$-axis indicate the percentiles at which RTs to redundant targets were reliably faster than corresponding RTs for the sum of the single CDFs.

single alone" $\mathrm{CDF}$ at the 5th, 15th, 25th, and 35th percentiles $(.001<p<.02)$. This shows that the fastest responses to redundant targets can in fact be faster than the fastest response to single targets, which is inconsistent with predictions of all race models. Analogously, we compared the redundant-target CDF to the sum of the CDFs in the "single target + neutral" condition to test for a violation of the race-model inequality when single targets are combined with neutral events (lower panel in Figure 4). As is apparent from this figure, the race-model inequality was again considerably violated. RTs were reliably faster for the redundant-target $\mathrm{CDF}$ at percentiles $0.05,0.15$, $0.25,0.35,0.45,0.55$, and $0.65(.001<p<.005)$.

The results argue against separate and independent processing of color and motion signals in a speeded target detection task with moving RDPs. We interpret this finding as evidence for cross-dimensional integration of color and motion information. In Experiment 1, neither the examination of redundancy gains nor the test for violations of the race-model inequality revealed any difference depending on whether we used single signals alone or single signals combined with neutral events. This finding is inconsistent with the idea that redundancy gains in the "single target + neutral" condition are simply due to response interference or cognitive inhibition effects. For instance, one could conceive that participants internally define the stimuli such that the designated color and direction were labeled as "go signals" and all others were considered "nogos." Resolving this conflicting information might have slowed down responses in the "single target + neutral" condition, thereby artificially producing a redundant-target effect (RTE), because redundant targets never contained conflicting information. However, because there is no difference between responses to single targets and single targets combined with neutral events (Figure 3), we can safely reject this possibility. Having demonstrated that adding a neutral signal to a single target has no inhibitory effect, we did not include the "single target alone" condition in all the succeeding experiments (i.e., we only used single targets that were combined with neutral events).

\section{Experiment 2}

The purpose of Experiment 2 was to investigate whether the observed cross-dimensional integration of color and motion signals requires that the two target signals belong to the same object. We rotated two populations of dots against each other, resulting in the percept of two superimposed surfaces (i.e., objects). This enabled us to present redundant targets always at the same location but either on the same surface or on different surfaces. If color and motion signals are integrated only if they belong to the same object, then we should observe violations of the race-model inequality only if redundant targets are presented on the same surface but not if they are presented on different surfaces.

\section{Methods}

The basic go/no-go target detection paradigm was identical to Experiment 1 except for the following changes. First, two RDPs were rotated against each other (Figure 1b). Second, because of superimposing two RDPs, there were twice as many dots in the transparent motion stimulus (4 dots/ $/ \mathrm{deg}^{2}$ of visual angle) as compared with the single surface used in Experiment 1. Third, single targets in a given stimulus dimension were always combined with neutral events in the other dimension. Fourth, six blocks 


\begin{tabular}{|c|c|c|c|c|c|c|c|}
\hline & \multicolumn{3}{|c|}{ Go signals } & \multicolumn{3}{|c|}{ No-go signals } \\
\hline & & Redundant & Single color & Single direction & Redundant & Single color & Single direction \\
\hline \multirow[t]{2}{*}{ Same surface } & Surface 1 & Red + right & Red + up & Right + blue & Green + left & Green + up & Left + blue \\
\hline & Surface 2 & No change & No change & No change & No change & No change & No change \\
\hline \multirow[t]{2}{*}{ Different surfaces } & Surface 1 & Red & Red & Right & Green & Green & Left \\
\hline & Surface 2 & Right & Up & Blue & Left & Up & Blue \\
\hline
\end{tabular}

Table 2. Experiment 2. One possible combination of go-, no-go, and neutral events for participants instructed to detect rightward motion (direction target) or the color red (color target). Single color signals were always accompanied by a neutral change in direction (upward motion); single direction signals were always presented together with a neutral change in color (blue). All events occurred either on the same surface or on different surfaces.

consisting of 120 trials each were run on two consecutive days. In a single block, there were 12 signal conditions: 6 go conditions comprising redundant targets with both signals in the same surface or in different surfaces, single color target + neutral direction event in the same surface or in different surfaces, and single direction target + neutral color event in the same surface or in different surfaces and also 6 no-go conditions comprising redundant no-gos in the same surface or in different surfaces, single color no-go + neutral direction event in the same surface or in different surfaces, and single direction no-go + neutral color event in the same surface or in different surfaces. See Table 2 for an overview of a possible combination of go, no-go, and neutral events in Experiment 2. Twelve participants (five men and seven women; ages $20-33, M=24.9, S D=3.4$ ) participated in Experiment 2. They were informed that the changes in color and direction of motion could either occur in the same surface or in different surfaces.

\section{Data analyses}

As before, redundancy gains were examined by comparing RTs to redundant targets with the fastest RTs to single targets. Mean RTs were computed for each combination of participant, target type (redundant target vs. single target), and target surface (same vs. different). The average of the faster of the two single-target conditions was calculated across participants, separately for each target surface condition. Statistical significance was evaluated with a twoway ANOVA involving the within-subjects factors target type (redundant target vs. "fastest single target") and target surface (same vs. different). Violations of the race-model inequality were evaluated as in Experiment 1.

\section{Results and discussion}

\section{Redundancy gain}

Across participants, performance reached $92 \%$ correct for the no-go trials and varied between $98 \%$ and $100 \%$ correct for the five go-conditions. Mean RTs across participants are shown in Figure 5. The ANOVA revealed a highly significant main effect of target type, $F(1,11)=$ 53.68, $M S E=192.67, p<.001$, indicating that responses to redundant targets were reliably faster than responses to the single targets (326 vs. $355 \mathrm{~ms}$ ). A $95 \%$ confidence interval for the main effect of target type indicates that redundancy gains are in the range of $20-38 \mathrm{~ms}$. Most importantly, neither the main effect of target surface $(p=.17)$ nor the interaction between target type and target surface $(p=.87)$ reached significance. This clearly shows that there is no difference in redundancy gains between changes occurring in the same surface $(29 \mathrm{~ms})$ and changes occurring in different surfaces $(30 \mathrm{~ms})$ in a transparent motion stimulus.

\section{Race-model inequality}

Mean CDFs across participants are shown in Figure 6. The upper panel represents conditions in which changes in color, direction of motion, or both occur in the same surface of a transparent motion stimulus. Contrasting the redundant-target CDF (pentagrams) with the "sum of single targets" CDF (circles) reveals a clear violation of the race-model inequality because the redundant-target CDF lies above and to the left of the "sum of single targets" CDF. Paired $t$ tests across participants at each of the 10 percentile

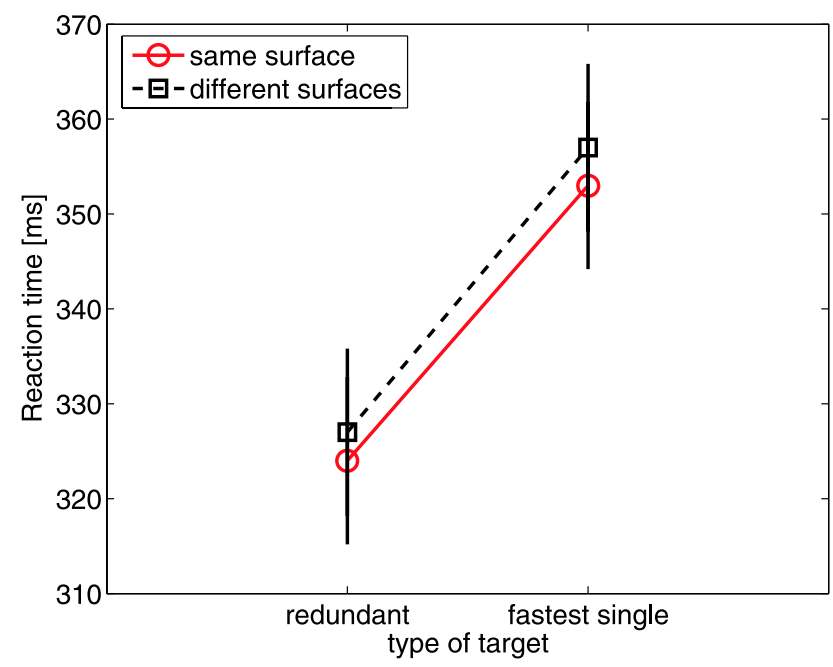

Figure 5. Experiment 2. RTs as a function of target type (redundant target vs. fastest single target) and target surface (same surface vs. different surfaces). RTs to redundant targets are faster than RTs to single targets. This effect does not depend on whether redundant targets are presented on the same surface or on different surfaces. Error bars indicate the $95 \%$ confidence interval for the main effect of target type (see Loftus \& Masson, 1994). 

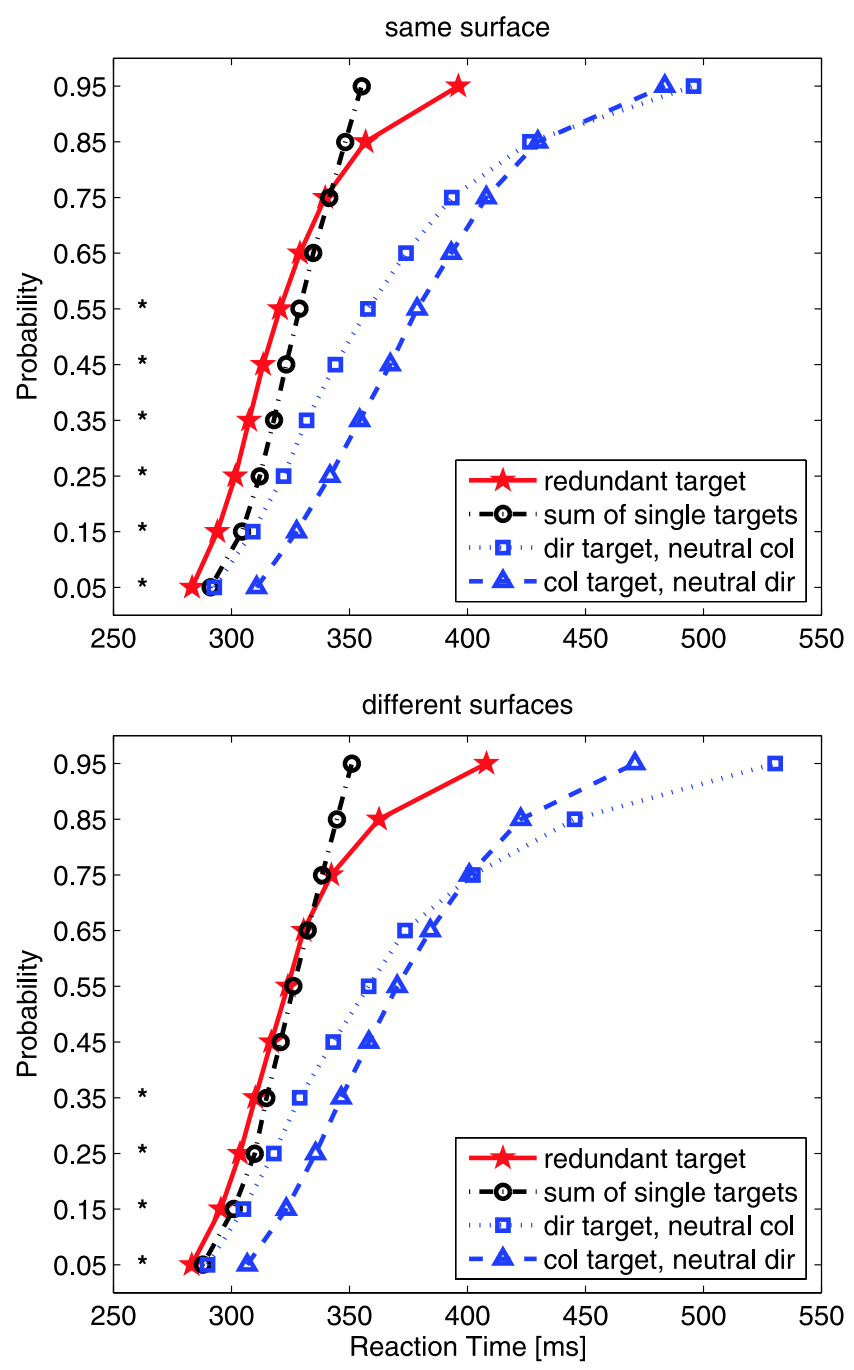

Figure 6. Experiment 2. Violations of the race-model inequality are revealed by comparing the redundant-target CDF (pentagrams) with the sum of the single-target CDFs (circles). Inconsistent with predictions of all race models, the redundant-target CDF lies above and to the left of the CDF for the sum of the single targets. This is the case not only if redundant targets occur on the same surface (upper panel) but also if they appear on different surfaces (lower panel). Significance markers along the $Y$-axis indicate the percentiles at which RTs to redundant targets were reliably faster than corresponding RTs for the sum of the single CDFs.

pairs confirmed that RTs from the redundant-target CDF were reliably faster than RTs from the "sum of single targets" CDF between the 5th and 55th percentiles $(.001<p<.002)$. Analogously, we compared the redundant-target CDF to the "sum of single targets" CDF in the condition where changes in color, direction of motion, or both occurred in different surfaces (lower panel in Figure 6). The race-model inequality was again considerably violated. RTs were reliably faster for the redundant-target $\mathrm{CDF}$ between percentiles 0.05 and $0.35(.01<p<.02)$.
The results of Experiment 2 show that color and motion signals are integrated, even if they occur in different surfaces of a transparent motion paradigm. This is evident from the fact that the magnitude of redundancy gains does not depend on whether two target signals occur in the same surface or in different surfaces. In addition, violations of the race-model inequality are prominent in both situations, suggesting integration of color and motion information across overlapping surface borders. Alternatively, one might argue that these two signals are integrated simply because they occur at the same location. Next, we tested whether integration of color and motion signals persists even if the two surfaces are positioned at different spatial locations.

\section{Experiment 3}

The purpose of Experiment 3 was to investigate whether the integration of color and motion signals across surfaces requires that the two surfaces are spatially overlapping. Instead of superimposing the two RDPs, we separated them spatially (Figure 1). If integration is restricted to a common spatial location, we should observe violations of the race-model inequality only if the target signals are presented at the same location but not if they are presented at different locations.

\section{Methods}

The basic go/no-go target detection paradigm was identical to Experiment 2 except for the following changes. The two RDPs were presented at spatially separate locations, centered 3.75 deg above and below fixation. Each of the 12 signal conditions was presented 12 times (6 go conditions: redundant targets with both signals in the same location or in different locations, single color target + neutral direction event in the same location or in different locations, and single direction target + neutral color event in the same location or in different locations; 6 no-go conditions: redundant no-go in the same location or in different locations, single color no-go + neutral direction event in the same location or in different locations, and single direction no-go + neutral color event in the same location or in different locations). Within each condition, three trials were used for each possible combination of the upper RDP's initial direction of rotation (clockwise vs. counterclockwise) and the location of the target event (upper vs. lower RDP). See Table 3 for a possible combination of go, no-go, and neutral events. Twelve participants (six men and six women; ages $20-27, M=23, S D=2.6$ ) were tested in Experiment 3. They were instructed to fixate on the central fixation square during the trials. Throughout each session, the experimenter monitored eye fixation with an infrared camera connected to a monitor outside the testing booth. 


\begin{tabular}{|c|c|c|c|c|c|c|c|}
\hline & \multicolumn{3}{|c|}{ Go signals } & \multicolumn{3}{|c|}{ No-go signals } \\
\hline & & Redundant & Single color & Single direction & Redundant & Single color & Single direction \\
\hline \multirow[t]{2}{*}{ Same location } & Location 1 & Red + right & Red + up & Right + blue & Green + left & Green + up & Left + blue \\
\hline & Location 2 & No change & No change & No change & No change & No change & No change \\
\hline \multirow[t]{2}{*}{ Different locations } & Location 1 & Red & Red & Right & Green & Green & Left \\
\hline & Location 2 & Right & Up & Blue & Left & Up & Blue \\
\hline
\end{tabular}

Table 3. Experiment 3. One possible combination of go-, no-go, and neutral events for participants instructed to detect rightward motion (direction target) or the color red (color target).

\section{Data analyses}

Redundancy gains were examined as before. Mean RTs were determined for each combination of participant, target type (redundant target vs. single target), and target location (same vs. different). For each participant, the faster of the two mean RTs in the single-target conditions was selected ("fastest single same" and "fastest single different," respectively). Statistical significance was evaluated with a two-way ANOVA involving the within-subjects factors target type (redundant target vs. fastest single target) and target location (same vs. different).

\section{Results and discussion}

\section{Redundancy gain}

Across participants, performance reached 97\% correct for the no-go trials and varied between $96 \%$ and $100 \%$ correct for the five go-conditions. Mean RTs across participants are shown in Figure 7. The ANOVA revealed a

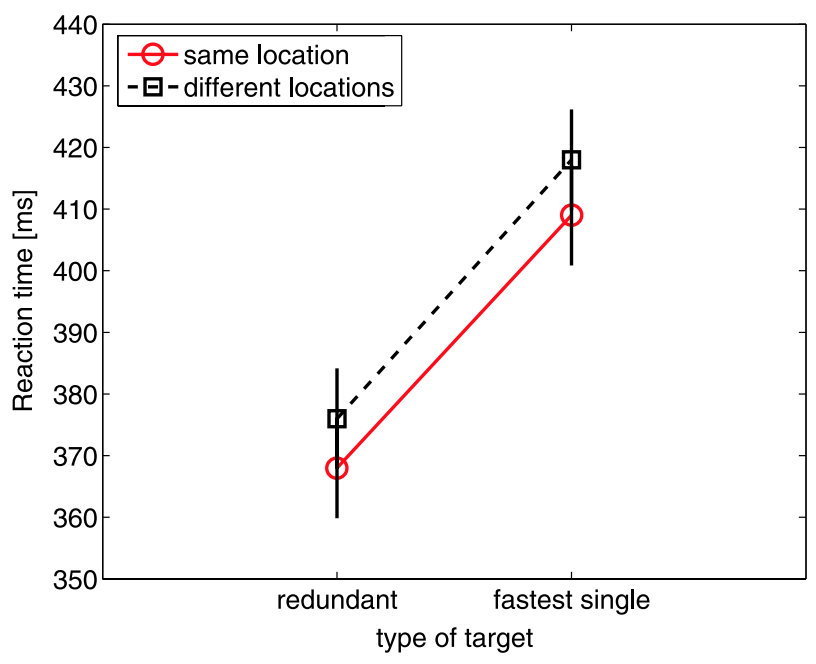

Figure 7. Experiment 3. RTs as a function of target type and target location. RTs to redundant targets are faster than RTs to single targets. RTs are also faster for events occurring at the same location than for those occurring at different locations. The absence of a significant interaction shows that the RTE does not depend on whether redundant targets are presented at the same location or at different locations. Error bars indicate the 95\% confidence interval for the main effect of target type (see Loftus \& Masson, 1994). highly significant main effect of target type, $F(1,11)=$ $125.74, M S E=165.08, p<.001$, indicating that responses to redundant targets $(372 \mathrm{~ms})$ were reliably faster than responses to the single targets $(414 \mathrm{~ms})$. A $95 \%$ confidence interval for the main effect of target type indicates that redundancy gains are in the range of 34-50 ms. The main effect of target location also reached significance, $F(1,11)=$ $7.15, M S E=118.51, p<.05$, indicating that responses to targets presented at the same location $(389 \mathrm{~ms})$ were faster than to targets presented at different locations (397 ms). Most important, however, was the absence of an interaction between target type and target location $(p=.8)$. This clearly shows that redundancy gains do not depend on whether the target events are presented at the same location (41 $\mathrm{ms})$ or at different locations (42 ms).

\section{Race-model inequality}

Mean CDFs across participants are shown in Figure 8. The upper panel represents conditions in which changes in color, direction of motion, or both occur at the same location. Paired $t$ tests across participants at each of the 10 percentile pairs confirmed that RTs from the redundanttarget CDF (pentagrams) were reliably faster than RTs from the "sum of single targets" CDF (circles) everywhere between the 5th and 55th percentiles $(.001<p<.05)$. The same comparison for the condition in which the target events occurred at different locations (lower panel in Figure 7) also revealed faster RTs for the redundant-target CDF everywhere between percentiles 0.05 and $0.65(.001<p<.05)$. Hence, in both cases, strong violations of the race-model inequality were observed.

These results show that redundancy gains and violations of the race-model inequality are prominent even if the two target events occur at separate spatial locations. It provides evidence for integration of color and motion signals across the visual field. To further assess the generality of this conclusion, we next tested whether the observed effects depend on the extent of spatial separation between the two stimuli.

\section{Experiment 4}

The purpose of Experiment 4 was to investigate whether the cross-dimensional integration observed in Experiment 3 is affected by the spatial distance between two RDPs. 

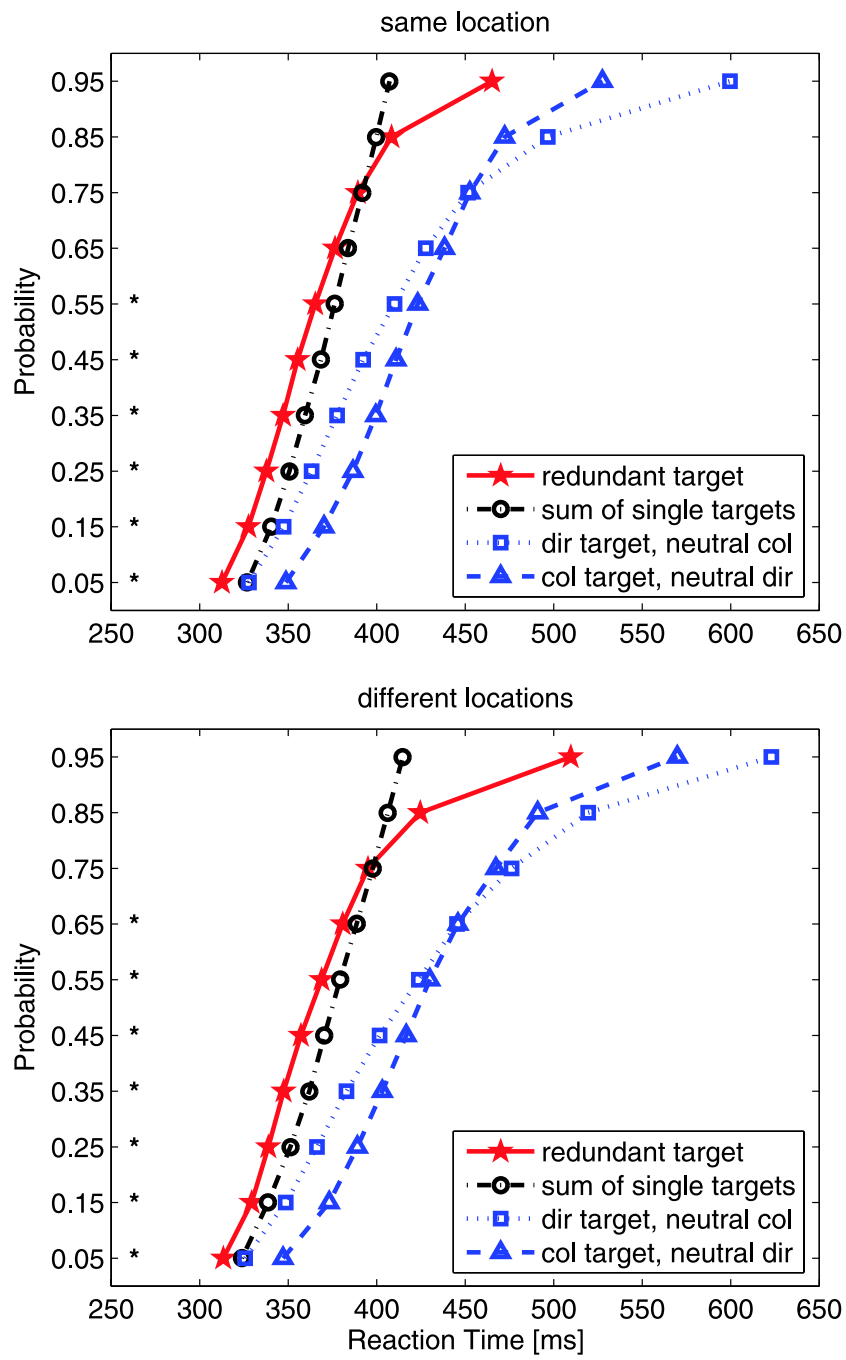

Figure 8. Experiment 3. Violations of the race-model inequality are revealed by comparing the redundant-target CDF (pentagrams) with the sum of the single-target CDFs (circles). Inconsistent with predictions of all race models, the redundant-target CDF lies above and to the left of the CDF for the sum of the single targets. This is evident irrespective of whether redundant targets occur at the same location (upper panel) or at different locations (lower panel). Significance markers along the $Y$-axis indicate the percentiles at which RTs were reliably faster for redundant targets than for the sum of single targets.

\section{Methods}

Methods and design were identical to Experiment 3 except for the spatial distance between the two RDPs, which were centered $7.5 \mathrm{deg}$ above and below fixation here (Figure 1c). To achieve this, we reduced the distance between participant and monitor to $57 \mathrm{~cm}$, resulting in a monitor resolution of 40 pixels/deg of visual angle. All stimulus properties were adjusted to ensure identity of the retinal image. Twelve participants (five men and seven women; ages $22-32, M=24.2, S D=2.9$ ) were tested in Experiment 4.
Redundancy gains and violations of the race-model inequality were evaluated as in the preceding experiments.

\section{Results and discussion}

\section{Redundancy gain}

Across participants, performance reached $97 \%$ correct for the no-go trials and varied between $92 \%$ and $100 \%$ correct for the five go-conditions. Mean RTs across participants are shown in Figure 9.

The ANOVA revealed a highly significant main effect of target type, $F(1,11)=159.18, M S E=100.50, p<.001$, indicating that responses to redundant targets were reliably faster than responses to the single targets (412 vs. $448 \mathrm{~ms}$ ). A 95\% confidence interval for the main effect of target type indicates that redundancy gains are in the range of 30-42 ms. The main effect of target presentation also reached significance, $F(1,11)=14.58, M S E=$ $178.27, p<.01$, indicating that responses to targets presented in the same location were faster than to targets presented in different locations (423 vs. $438 \mathrm{~ms}$ ). However, as in Experiment 3, the interaction between target type and target location did not reach significance $(p=.14)$, indicating that there is no difference in redundancy gains between changes in the same location ( $38 \mathrm{~ms}$ ) and changes in different locations $(31 \mathrm{~ms})$. It clearly shows that the magnitude of the redundancy gains is not affected by the spatial distance between the two stimuli. This conclusion is further supported by Miller (1982), who also reported

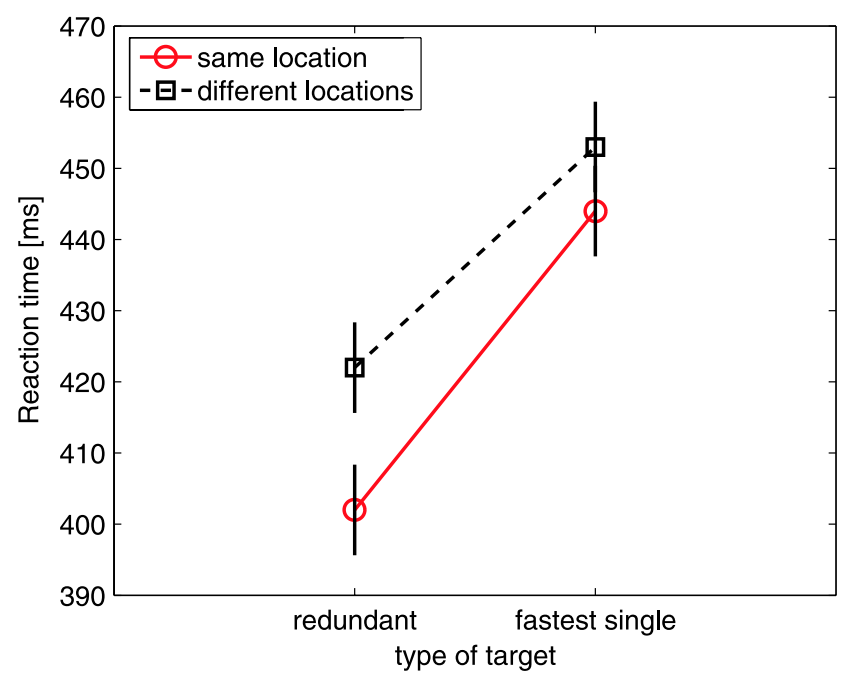

Figure 9. Experiment 4. RTs as a function of target type and target location. RTs to redundant targets are faster than RTs to single targets. RTs are also faster for events occurring in the same location than for those occurring in different locations. The absence of a significant interaction shows that the RTE does not depend on whether redundant targets are presented in the same location or in different locations. Error bars indicate the 95\% confidence interval for the main effect of target type (see Loftus \& Masson, 1994). 
the absence of distance effects in a different variant of the redundant-target paradigm.

\section{Race-model inequality}

Mean CDFs across participants are shown in Figure 10. Conditions in which changes in color, direction of motion, or both occur at the same location are shown in the upper panel. Paired $t$ tests across participants at each of the 10 percentile pairs confirmed that RTs from the redundant-
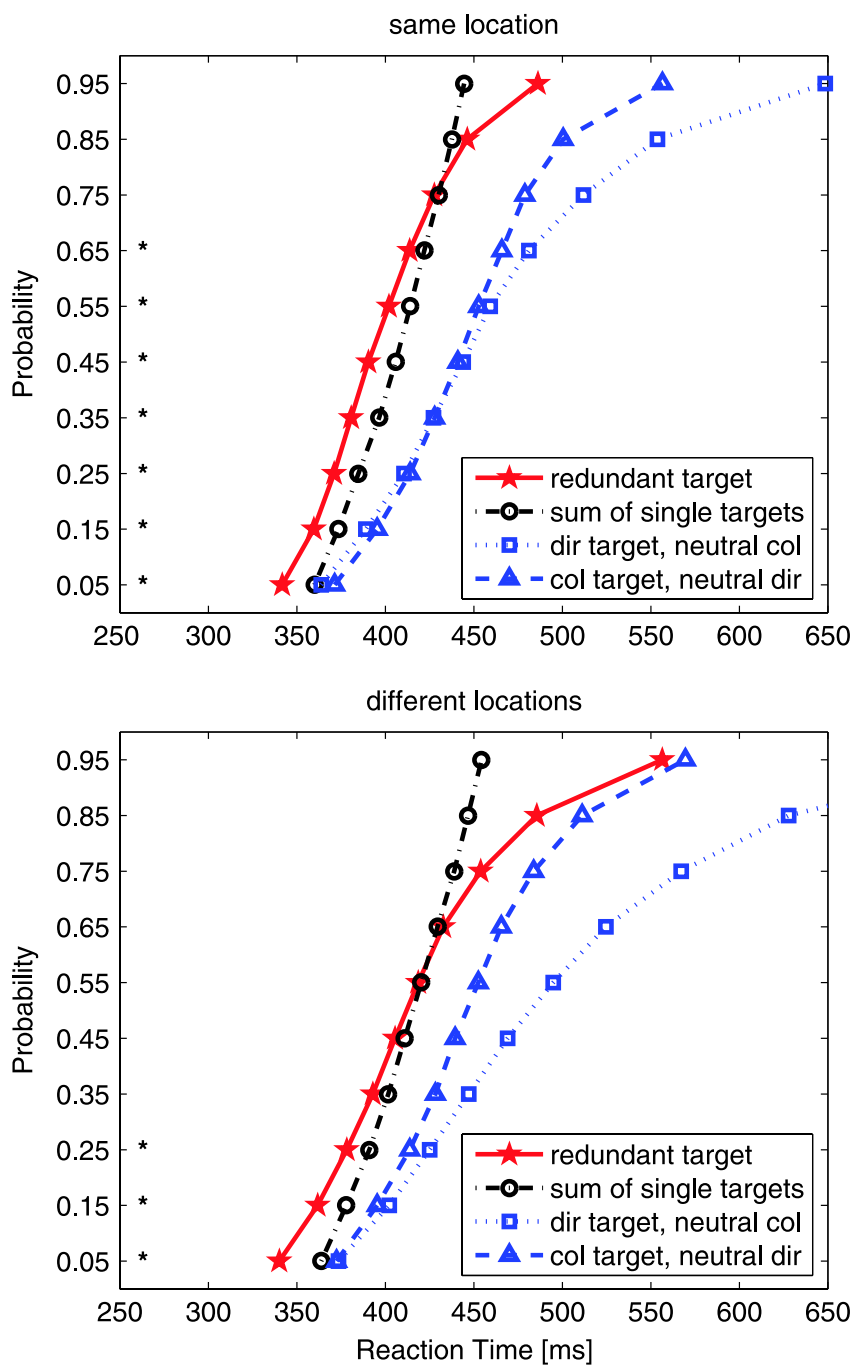

Figure 10. Experiment 4. Violations of the race-model inequality revealed by comparing the redundant-target CDF (pentagrams) with the sum of the single-target CDFs (circles). Inconsistent with predictions of all race models, the redundant-target CDF lies above and to the left of the CDF for the sum of the single targets. This is evident irrespective of whether redundant targets occur at the same location (upper panel) or at different locations (lower panel). It shows that the fastest response to redundant targets can be faster than the fastest responses to single targets. Significance markers along the $Y$-axis indicate the percentiles at which RTs were reliably faster for redundant targets than for the sum of single targets. target CDF (pentagrams) were reliably faster than RTs from the "sum of single targets" CDF (circles) everywhere between the 5th and 65th percentiles $(.001<p<.008)$. The lower panel of Figure 10 shows the condition in which the target events occurred at different locations. RTs for the redundant-target $\mathrm{CDF}$ were significantly faster between percentiles 0.05 and $0.25(.001<p<.01)$, with the difference at the 35 th percentile just closely failing to reach statistical significance $(p=.057)$. Hence, for targets at the same and at distant locations, strong violations of the race-model inequality were observed.

The outcome of Experiment 4 further supports the notion that color and motion information is integrated across stimuli presented at different locations in the visual field. Even when the stimuli are separated by 15 deg (center-tocenter distance), strong redundancy gains and reliable violations of the race-model inequality are observed.

\section{General discussion}

In four experiments, we investigated the cross-dimensional integration of color and visual motion signals by using the redundant-target paradigm. We presented moving RDPs in a speeded go/no-go target detection task, in which participants were required to respond to changes in the direction of motion (single target), color (single target), or both (redundant target). Experiment 1 established that redundancy gains are present for targets defined by their direction of visual motion and color: Responses to redundant targets were faster than responses to single targets. There was no difference between single targets presented alone and single targets that were combined with neutral events in the other stimulus dimension, which argues against the possibility that our redundancy gains are caused by inhibitory influences due to the presentation of a neutral event. Furthermore, the redundancy gains were inconsistent with predictions based on race models. We consider this as evidence that color and motion signals are integrated rather than processed separately and independently in this particular task. Experiment 2 shows that such integration is not restricted to a single surface in a transparent motion paradigm. Whereas redundant targets were presented either in the same surface or in different surfaces, integration of color and motion signals was revealed in both cases. Finally, Experiments 3 and 4 show that the integration of target information is independent of the spatial separation between the stimuli: Integration was observed irrespective of whether redundant targets were presented at the same location or at different locations, even if the spatial distance between the stimuli was as large as 15 deg. Taken together, this series of experiments demonstrates the integration of visual features that are represented in distinct visual areas across different stimulus constellations and task demands.

Our data allow us to draw conclusions about potential mechanisms of attention underlying performance in the 
redundant-target paradigm. Essentially, three different types of attentional mechanisms have been reported. Spatial attention refers to the ability to attend to a particular location in the visual field. All sensory information presented at the attended location is processed more efficiently than information outside the spatial focus of attention (Eriksen \& St. James, 1986; Posner, 1980). Feature-based attention, on the other hand, describes a mechanism by which attending to a particular feature (i.e., the color "red") enhances processing of only the attended feature, independent of the spatial focus of attention. Finally, object-based attention means that attention can be directed to perceptual groups or "objects" and that features of the same object are processed more efficiently than features belonging to different objects ("same-object advantage"; Blaser, Pylyshyn, \& Holcombe, 2000; Duncan, 1984; O’Craven, Downing, \& Kanwisher, 1999; for reviews, see Driver \& Baylis, 1998; Scholl, 2001). Strong evidence for object-based attentional mechanisms comes from studies in which two objects are superimposed, such that spatial location by itself cannot be used to orient attention. As for visual motion, superimposed objects are created by overlaying two RDPs moving coherently in opposite directions, thereby generating the percept of two surfaces sliding across each other. By assuming an objectbased mechanism of visual attention, one would predict that redundancy gains and violations of the race-model inequality should be obtained only for redundant targets occurring on the same but not on different surfaces (sameobject advantage). In contrast, we find strong redundancy gains and reliable violations of the race-model inequality in both conditions (Experiment 2), ruling out an object-based account of the effect. By assuming a spatial attentional mechanism, on the other hand, one would expect that redundancy gains and race-model violations should become evident for redundant targets occurring at the same location but not for different locations. However, the effects were consistently independent of spatial location (Experiments 3 and 4). Therefore, we propose that a feature-based attentional mechanism can best account for our findings. Several studies have demonstrated that observers can effectively attend to nonspatial stimulus features, and this has also been shown for color (Anllo-Vento \& Hillyard, 1996; Corbetta, Miezin, Dobmeyer, Shulman, \& Petersen, 1990; Sàenz, Buracas, \& Boynton, 2003) and direction of motion (Martínez-Trujillo \& Treue, 2004; Sàenz, Buracas, \& Boynton, 2002; Treue \& MartínezTrujillo, 1999; Treue \& Maunsell, 1996). In our task, participants have divided their attention between the target color and target direction, resulting in an enhanced processing of the designated target features throughout the visual field. The RTE has already been related to featurebased attention in the study by Mordkoff and Yantis (1993). In their variant of the redundant-target paradigm, they investigated integration of color and shape information and found violations of the race-model inequality not only if the two target elements were part of the same object (i.e., a colored letter) but also if they occurred at different spatial locations (i.e., a colored frame around a white letter or a colored patch below the letter). Yet, there are two important differences between these experiments and our approach. First, using moving RDPs allowed us to superimpose two objects and randomly present same-object and differentobject conditions without any change in stimulus attributes or perceptual task. Second, whereas Mordkoff and Yantis have demonstrated violations of the race-model inequality for the stimulus dimensions color and shape, we show such violations for the dimensions color and direction of motion.

At first sight, our results might seem to be difficult to reconcile with recent behavioral studies underscoring the importance of location, on the one hand, or objects, on the other hand, for performance in visual tasks. In particular, there are two different lines of research that both arrive at apparently discrepant conclusions: investigations of feature binding and work on object-based visual attention. Investigating feature binding, Holcombe and Cavanagh (2001), for example, have demonstrated that the stimulus dimensions color and orientation are correctly bound at very high rates of presentation but only if they were superimposed (i.e., presented at the same location). Why does location matter in the case of feature binding but not in our paradigm? We think that this discrepancy can be explained by differences in task demands. As Roskies (1999) has framed it, for feature binding, "one sort of visual feature [...] must be correctly associated with another feature [...] to provide a unified representation of that object." Such an explicit association is not a requirement in the type of divided-attention paradigm we have employed. In fact, because the two target features are not necessarily presented together in a given trial (single targets), an explicit association of the target features is impossible in those trials. Instead, detection of either target feature (direction of motion or color) is sufficient to initiate a response: As soon as, for example, the color red appears, a speeded response is required, regardless of the direction the red dots are moving. Likewise, the presence of, for example, rightward motion calls for an immediate response, irrespective of the color of the rightward moving dots. If we had intended to investigate the binding of color and motion, we would have asked the participants to indicate, for example, in which direction the red dots were moving. In such a situation, we would certainly expect location-based effects, that is, better performance if these two features were presented in the same location as compared with different locations. In our task, however, examining in which direction the red dots are moving might even have detrimental effects on RT. In the second line of research, transparent motion paradigms have been used to investigate object-based mechanisms of visual attention. Simultaneous judgments about speed and direction of motion are more accurate if they concern the same surface as opposed to different surfaces (Valdés-Sosa, Cobo, \& Pinilla, 1998). Furthermore, when a cue directs attention to one of two superimposed surfaces, subsequent changes in the direction of motion are discriminated less accurately in the uncued surface compared with the cued one (Mitchell, Stoner, Fallah, \& Reynolds, 2003; Reynolds, 
Alborzian, \& Stoner, 2003; Valdés-Sosa, Cobo, \& Pinilla, 2000). The underlying electrophysiological mechanism seems to be a selective reduction of visual neuronal responses to changes in the unattended surface, as evidenced by reduced early components of the event-related potential (ERP) (P1, N1; Valdés-Sosa, Bobes, Rodríguez, \& Pinilla, 1998). Given the considerable evidence for object-based attentional mechanisms with transparent motion stimuli, one might expect to find an indication of such mechanisms with the redundant-target paradigm as well. Just as in the case of feature binding, the object-based transparent motion studies we have mentioned place different demands on the visual system as compared with our task. Valdés-Sosa, Cobo, et al. (1998, 2000), Reynolds et al. (2003), and Mitchell et al. (2003) used designs that drew or directed attention to a given surface, resulting in prioritized processing of one surface over the other. Our design, in contrast, required participants to divide attention between the stimulus dimensions color and direction of motion, and not between two objects or surfaces. Because our targets were equally likely to appear in one surface or the other, participants would not benefit from allocating resources to one surface at the expense of the second one. Furthermore, as soon as the color turned, for example, red, a speeded response was required, regardless which surface was involved. To perform optimally in our task, participants would be well advised to just focus on, for example, the color red or some dots moving to the right and not to segregate the superimposed surfaces. Here as well, examining to which surface the target color belongs might be disadvantageous in terms of RT performance. Taken together, it seems justified to assume that differences in task demands can account for the apparent discrepancies between the various paradigms.

Alternatively, one could argue that these discrepancies might stem from the fact that we used speeded responses and compared RT distributions, whereas the other paradigms used threshold measurements (Holcombe \& Cavanagh, 2001) or percentage of correct responses (Reynolds et al., 2003; Valdés-Sosa et al., 2000) as their dependent variable. In particular, one might propose that feature binding (showing location-based effects) and attentional discrimination paradigms (showing object-based effects) directly probe mechanisms at early stages of visual processing, whereas RTs are influenced by many stages of processing between the retina and the motor cortex. However, it is not the case that RTs are insensitive to effects of perceptual integration and attention in early visual areas. For example, recent works on electrophysiological (Womelsdorf, Fries, Mitra, \& Desimone, 2005) and functional MRI (Weissman, Roberts, Visscher, \& Woldorff, 2005) have revealed a trial-by-trial correlation between stimulus-evoked activity in visual cortical areas and RT. Moreover, it has been shown that RTs also provide a signature for the presence or absence of feature binding, for example, in classic visual search studies (Treisman \& Gelade, 1980).

It is important for our approach, however, that the RTE is, at least partly, a perceptual effect and does not arise entirely at a premotor or motor level of processing. The locus of the RTE has been discussed controversially, with some studies advocating a premotor or motor contribution (e.g., Diederich \& Colonius, 1987; Giray \& Ulrich, 1993) but others arguing against it (Miller et al., 2001; Mordkoff et al., 1996). For instance, Giray and Ulrich (1993) measured response force in addition to RT. Response force, which is regulated in the motor cortex (Scott, 2003), was largest in redundant-target trials, leading the authors to propose contributions of motor areas to the RTE. However, Miller et al. (2001) provided direct evidence against a motor locus by analyzing single-cell recordings from primary motor cortex in nonhuman primates. Briefly, if the RTE originated during perceptual processing, the input signals to the motor cortex should already be speeded in redundant-target trials. This would be evident in shorter latencies (i.e., time differences between stimulus onset and onset of neuronal activity) of primary motor neurons in response to redundant targets compared with single targets. Alternatively, if the RTE arose at late motor levels of processing, the motor cortex output signals should show an additional redundancy gain. In this case, the difference between response latencies of primary motor neurons and corresponding RTs should be smaller for redundant trials compared with single signal trials. Miller et al. found a reduction in neuronal response latencies to redundant targets, although there was no difference between neuronal latencies and RTs directly disconfirming the hypothesis that late motor areas constitute the origin of the RTE. Moreover, evidence in favor of perceptual contributions to the RTE has been reported consistently by a number of studies. ERP recordings have demonstrated influences of redundant targets on components associated with early visual processing (N1, P1; Miniussi et al., 1998) or target selection (P2, N2p; Reimann, Müller, \& Krummenacher, 2004). Behaviorally, early sensory or perceptual contributions to the RTE have also been reported by Turatto et al. (2004) and Krummenacher et al. (2002), respectively. Taken together, these studies confirm that there is a substantial perceptual component to the RTE.

The functional integration of color and motion information has been investigated in a number of other paradigms, using behavioral, neurophysiological, and neuroimaging methods. Evidence for mostly independent processing of color and motion comes from psychophysical studies on temporal asynchronies in visual perception (Arnold \& Clifford, 2002; Arnold, Clifford, \& Wenderoth, 2001; Moutoussis \& Zeki, 1997; Nishida \& Johnston, 2002; Viviani \& Aymoz, 2001). Moutoussis and Zeki (1997) used moving objects that change their direction of motion and color. Although these changes would occur in perfect synchrony in some trials, they were shifted by different time lags with respect to each other in other trials. Psychophysical measures of the point of subjective synchrony revealed that motion changes have to happen $70-80 \mathrm{~ms}$ earlier than color changes for them to be perceived as occurring simultaneously. Exploiting the color-contingent motion aftereffect 
as an indirect measure of perceptual synchrony, Arnold et al. (2001) demonstrated a similar amount of processing lag for color and motion attributes of a stimulus. Such asynchronies in perception are taken as evidence for independent processing of color and motion signals and for a functional specialization of the visual brain areas (Zeki \& Bartels, 1998, but see also Bedell, Chung, Ogmen, \& Patel, 2003). In contrast, a number of studies have demonstrated functional interactions between the color and motion processing systems (for reviews, see Croner \& Albright, 1999a; Dobkins \& Albright, 1993a). If the two streams were functionally separate, the perception of a moving object should be impossible if object and background are isoluminant. This prediction has been rejected in behavioral experiments (Dobkins \& Albright, 1993b; Hawken, Gegenfurtner, \& Tang, 1994). Furthermore, corresponding neurophysiological studies have shown that neurons in the medial temporal area (MT), which is strongly implicated in the perception of visual motion (Salzman, Britten, \& Newsome, 1990; Salzman, Murasugi, Britten, \& Newsome, 1992), continue to signal the direction of motion of heterochromatic stimuli even under conditions of isoluminance (Dobkins \& Albright, 1994; Gegenfurtner et al., 1994; Saito, Tanaka, Isono, Yasuda, \& Mikami, 1989). Similarly, it has been demonstrated that color information can improve perceptual performance: Psychometric and neurometric detection thresholds in coherent motion displays are strongly reduced if dots carrying the motion signal and random noise can be segmented based on different but isoluminant colors (Croner \& Albright, 1997, 1999b). The chromatic influence on motion processing can be strong, particularly under conditions of low luminance contrast (Thiele, Dobkins, \& Albright, 1999, 2001), and is independent of attentional load (Thiele, Rezec, \& Dobkins, 2002). Whereas some studies have shown a more prominent contribution of color information to motion processing for stimuli modulated along the red-green cardinal axis in color space (i.e., with L- and M-cone input; Gegenfurtner et al., 1994; Ruppertsberg, Wuerger, \& Bertamini, 1993, but see also Lu, Lesmes, \& Sperling, 1999 for an alternative account), others have also documented reliable effects for stimuli modulated along the yellowish-violet axis (i.e., with S-cone input; Seidemann \& Newsome, 1999; Wandell et al., 1999). Taken together, these studies demonstrate that color information is available to the visual motion processing system. Likewise, it has been shown that some neurons in the ventral stream area V4, which is mainly specialized for the processing of orientation and color, are direction selective (Desimone \& Schein, 1987; Ferrera, Rudolph, \& Maunsell, 1994; Mountcastle, Motter, Steinmetz, \& Sestokas, 1987; Tolias, Keliris, Smirnakis, \& Logothetis, 2005). Together, these findings provide strong evidence for shared neuronal resources for color and motion processing across the two visual streams, potentially representing a neural substrate for the perceptual integration of color and visual motion signals.

Possibly, one could object that perceptual integration of color and motion signals is achieved entirely by dorsal stream processing in the present experiments. Although the colors used in our experiments were objectively isoluminant $\left(25 \mathrm{~cd} / \mathrm{m}^{2}\right)$, there might have been differences in perceived, subjective isoluminance, which might have been driving dorsal stream neurons. We argue that such an effect cannot explain the integration observed. In all experiments, four different colors were used (gray, red, green, and blue), of which only one was the designated target color. Thus, participants could not simply respond to changes in chromatic contrast or possible differences in subjective isoluminance. Considering the extremely low error rate, it seems highly unlikely that participants based their perceptual decisions on differences in subjective luminance, which, presumably, would have been much smaller than the differences in chromatic contrast. Moreover, if the dorsal stream simply integrated changes in direction of motion with changes in subjective isoluminance, no difference between redundant targets and direction targets combined with neutral color changes would be expected. In fact, our data demonstrate highly significant differences between these two conditions, ruling out such an interpretation of the results.

In conclusion, we demonstrate the integration of color and motion information in a speeded detection task using the redundant-target paradigm. All variants of race models proposing independent and separate processing of color and motion signals in such a task can be ruled out. This is in line with recent psychophysical and neurophysiological evidence for substantial interactions between the color and motion processing systems. We show that this integration persists throughout different stimulus constellations and task demands. With transparent motion stimuli, integration of color and motion signals occurs across overlapping object borders, ruling out object-based selection in such a design. Spatial separation of color and motion signals does not constrain their integration either, rejecting locationbased accounts of the effect. Feature-based theories of attentional selection, on the other hand, propose enhanced processing of the attended features throughout the visual field. Because color and motion signals neither have to be assigned to the same object nor to the same location to be integrated in the redundant-target paradigm, performance in this task can be best characterized by a feature-based mechanism of divided visual attention.

\section{Acknowledgments}

We thank Jeff Miller for valuable discussions and advice, Christoph Nothdurft for helpful comments on an earlier version of the manuscript, and Çaglar Akçay for testing the participants in Experiments 1 and 2.

Commercial relationships: none.

Corresponding author: Steffen Katzner.

Email: steffen.katzner@mail.gwdg.de.

Address: German Primate Center, Göttingen, Germany. 
References

Anllo-Vento, L., \& Hillyard, S. (1996). Selective attention to the color and direction of moving stimuli: Electrophysiological correlates of hierarchical feature selection. Perception \& Psychophysics, 58, 191-206. [PubMed]

Arnold, D. H., \& Clifford, C. W. (2002). Determinants of asynchronous processing in vision. Proceedings, Biological Sciences/The Royal Society, 269, 579-583. [PubMed]

Arnold, D. H., Clifford, C. W., \& Wenderoth, P. (2001). Asynchronous processing in vision: Color leads motion. Current Biology, 11, 596-600. [PubMed] [Article]

Bedell, H. E., Chung, S. T., Ogmen, H., \& Patel, S. S. (2003). Color and motion: Which is the tortoise and which is the hare? Vision Research, 43, 2403-2412. [PubMed]

Blaser, E., Pylyshyn, Z. W., \& Holcombe, A. O. (2000). Tracking an object through feature space. Nature, 408, 196-199. [PubMed]

Corbetta, M., Miezin, F. M., Dobmeyer, S., Shulman, G. L., \& Petersen, S. E. (1990). Attentional modulation of neural processing of shape, color, and velocity in humans. Science, 248, 1556-1559. [PubMed]

Croner, L. J., \& Albright, T. D. (1997). Image segmentation enhances discrimination of motion in visual noise. Vision Research, 37, 1415-1427. [PubMed]

Croner, L., \& Albright, T. D. (1999a). Seeing the big picture: Integration of image cues in the primate visual system. Neuron, 24, 777-789. [PubMed] [Article]

Croner, L., \& Albright, T. D. (1999b). Segmentation by color influences responses of motion-sensitive neurons in the cortical middle temporal visual area. The Journal of Neuroscience, 19, 3935-3951. [PubMed] [Article]

Desimone, R., \& Schein, S. J. (1987). Visual properties of neurons in area V4 of the macaque: Sensitivity to stimulus form. Journal of Neurophysiology, 57, 835-868. [PubMed]

Diederich, A., \& Colonius, H. (1987). Intersensory facilitation in the motor component? A reaction time analysis. Psychological Research, 49, 23-29.

Dobkins, K. R., \& Albright, T. D. (1993a). Color, luminance, and the detection of visual motion. Current Directions in Psychological Science, 2, 189-193.

Dobkins, K. R., \& Albright, T. D. (1993b). What happens if it changes color when it moves?: Psychophysical experiments on the nature of chromatic input to motion detectors. Vision Research, 33, 1019-1036. [PubMed]
Dobkins, K. R., \& Albright, T. D. (1994). What happens if it changes color when it moves?: The nature of chromatic input to macaque visual area MT. The Journal of Neuroscience, 14, 4854-4870. [PubMed] [Article]

Driver, J., \& Baylis, G. (1998). Attention and visual object segmentation. In R. Parasuraman (Ed.), The attentive brain (pp. 299-325). Cambridge, MA: MIT Press.

Duncan, J. (1984). Selective attention and the organization of visual information. Journal of Experimental Psychology: General, 113, 501-517. [PubMed]

Eriksen, C. W., \& St. James, J. D. (1986). Visual attention within and around the field of focal attention: A zoom lens model. Perception \& Psychophysics, 40, 225-240. [PubMed]

Felleman, D., \& Van Essen, D. C. (1991). Distributed hierarchical processing in the primate cerebral cortex. Cerebral Cortex, 1, 1-47. [PubMed]

Ferrera, V. P., Nealey, T. A., \& Maunsell, J. H. R. (1992). Mixed parvocellular and magnocellular geniculate signals in visual area V4. Nature, 358, 756-761. [PubMed]

Ferrera, V. P., Rudolph, K. K., \& Maunsell, J. H. (1994). Responses of neurons in the parietal and temporal visual pathways during a motion task. The Journal of Neuroscience, 14, 6171-6186. [PubMed] [Article]

Gegenfurtner, K. R., Kiper, D. C., Beusmans, J. M., Carandini, M., Zaidi, Q., \& Movshon, J. A. (1994). Chromatic properties of neurons in macaque MT. Visual Neuroscience, 11, 455-466. [PubMed]

Giray, M., \& Ulrich, R. (1993). Motor coactivation revealed by response force in divided and focused attention. Journal of Experimental Psychology: Human Perception and Performance, 19, 1278-1291. [PubMed]

Hawken, M. J., Gegenfurtner, K. R., \& Tang, C. (1994). Contrast dependence of colour and luminance motion mechanisms in human vision. Nature, 367, 268-270. [PubMed]

Holcombe, A. O., \& Cavanagh, P. (2001). Early binding of feature pairs for visual perception. Nature Neuroscience, 4, 127-128. [PubMed] [Article]

Iacoboni, M., \& Zaidel, E. (2003). Interhemispheric visuomotor integration in humans: The effect of redundant targets. The European Journal of Neuroscience, 17, 1981-1986. [PubMed]

Krummenacher, J., Müller, H. J., \& Heller, D. (2001). Visual search for dimensionally redundant pop-out targets: Evidence for parallel-coactive processing of dimensions. Perception \& Psychophysics, 63, 901-917. [PubMed]

Krummenacher, J., Müller, H. J., \& Heller, D. (2002). Visual search for dimensionally redundant pop-out targets: Redundancy gains in compound tasks. Visual Cognition, 90, 801-837. 
Loftus, G., \& Masson, M. (1994). Using confidence intervals in within-subjects designs. Psychonomic Bulletin \& Review, 1, 476-490.

Lu, Z. L., Lesmes, L. A., \& Sperling, G. (1999). The mechanism of isoluminant chromatic motion perception. Proceedings of the National Academy of Sciences of the United States of America, 96, 8289-8294. [PubMed] [Article]

Martínez-Trujillo, J. C., \& Treue, S. (2004). Feature-based attention increases the selectivity of population responses in primate visual cortex. Current Biology, 14, 744-751. [PubMed] [Article]

Miller, J. (1982). Divided attention: Evidence for coactivation with redundant signals. Cognitive Psychology, 14, 247-279. [PubMed]

Miller, J. (1986). Timecourse of coactivation in bimodal divided attention. Perception \& Psychophysics, 40, 331-343. [PubMed]

Miller, J. (2004). Exaggerated redundancy gain in the split brain: A hemispheric coactivation account. Cognitive Psychology, 49, 118-154. [PubMed]

Miller, J., Ulrich, R., \& Lamarre, Y. (2001). Locus of the redundant-signals effect in bimodal divided attention: A neurophysiological analysis. Perception \& Psychophysics, 63, 555-562. [PubMed]

Miniussi, C., Girelli, M., \& Marzi, C. A. (1998). Neural site of the redundant target effect electrophysiological evidence. Journal of Cognitive Neuroscience, 10, 216-230. [PubMed]

Mitchell, J., Stoner, G., Fallah, M., \& Reynolds, J. H. (2003). Attentional selection of superimposed surfaces cannot be explained by modulation of the gain of color channels. Vision Research, 43, 1323-1328. [PubMed]

Mordkoff, J. T., Miller, J., \& Roch, A. C. (1996). Absence of coactivation in the motor component: Evidence from psychophysiological measures of target detection. Journal of Experimental Psychology: Human Perception and Performance, 22, 25-41. [PubMed]

Mordkoff, J. T., \& Yantis, S. (1991). An interactive race model of divided attention. Journal of Experimental Psychology: Human Perception and Performance, 17, 520-538. [PubMed]

Mordkoff, J. T., \& Yantis, S. (1993). Dividing attention between color and shape: Evidence of coactivation. Perception \& Psychophysics, 53, 357-366. [PubMed]

Mountcastle, V. B., Motter, B. C., Steinmetz, M. A., \& Sestokas, A. K. (1987). Common and differential effects of attentive fixation on the excitability of parietal and prestriate (V4) cortical visual neurons in the macaque monkey. The Journal of Neuroscience, 7, 2239-2255. [PubMed] [Article]
Moutoussis, K., \& Zeki, S. (1997). A direct demonstration of perceptual asynchrony in vision. Proceedings: Biological Sciences / The Royal Society, 264, 393-399. [PubMed]

Nishida, S., \& Johnston, A. (2002). Marker correspondence, not processing latency, determines temporal binding of visual attributes. Current Biology, 12, 359-368. [PubMed] [Article]

O'Craven, K. M., Downing, P. E., \& Kanwisher, N. (1999). fMRI evidence for objects as the units of attentional selection. Nature, 401, 584-587. [PubMed]

Posner, M. (1980). Orienting of attention. The Quarterly Journal of Experimental Psychology, 32, 3-25. [PubMed]

Raab, D. H. (1962). Statistical facilitation of simple reaction times. Transactions of the New York Academy of Sciences, 24, 574-590. [PubMed]

Reimann, B., Müller, H. J., \& Krummenacher, J. (2004). Visual search for dimensionally redundant pop-out targets: Behavioral and electrophysiological evidence for parallel co-active processing and location specificity. 11th Annual Meeting of the Cognitive Neuroscience Society, San Francisco.

Reynolds, J. H., Alborzian, S., \& Stoner, G. (2003). Exogenously cued attention triggers competitive selection of surfaces. Vision Research, 43, 59-66. [PubMed]

Roskies, A. L. (1999). The binding problem. Neuron, 24, 7-9, 111-125. [PubMed] [Article]

Ruppertsberg, A. I., Wuerger, S. M., \& Bertamini, M. (1993). The chromatic input to global motion perception. Visual Neuroscience, 20, 421-428. [PubMed]

Sàenz, M., Buracas, G. T., \& Boynton, G. M. (2002). Global effects of feature-based attention in human visual cortex. Nature Neuroscience, 5, 631-632. [PubMed] [Article]

Sàenz, M., Buracas, G. T., \& Boynton, G. M. (2003). Global feature-based attention for motion and color. Vision Research, 43, 629-637. [PubMed]

Saito, H., Tanaka, K., Isono, H., Yasuda, M., \& Mikami, A. (1989). Directionally selective response of cells in the middle temporal area (MT) of the macaque monkey to the movement of equiluminous opponent color stimuli. Experimental Brain Research, 75, 1-14. [PubMed]

Salzman, C. D., Britten, K. H., \& Newsome, W. T. (1990). Cortical microstimulation influences perceptual judgements of motion direction. Nature, 346, 174-177. [PubMed]

Salzman, C. D., Murasugi, C. M., Britten, K. H., \& Newsome, W. T. (1992). Microstimulation in visual area MT: Effects on direction discrimination performance. The Journal of Neuroscience, 12, 2331-2355. [PubMed] [Article] 
Scholl, B. J. (2001). Objects and attention: The state of the art. Cognition, 80, 1-46. [PubMed]

Scott, S. H. (2003). The role of primary motor cortex in goal-directed movements: Insights from neurophysiological studies on non-human primates. Current Opinion in Neurobiology, 13, 671-677. [PubMed]

Seidemann, E., \& Newsome, W. T. (1999). Effect of spatial attention on the responses of area MT neurons. Journal of Neurophysiology, 81, 1783-1794. [PubMed] [Article]

Thiele, A., Dobkins, K. R., \& Albright, T. D. (1999). The contribution of color to motion processing in macaque middle temporal area. The Journal of Neuroscience, 19, 6571-6587. [PubMed] [Article]

Thiele, A., Dobkins, K. R., \& Albright, T. D. (2001). Neural correlates of chromatic motion perception. Neuron, 32, 351-358. [PubMed] [Article]

Thiele, A., Rezec, A., \& Dobkins, K. R. (2002). Chromatic input to motion processing in the absence of attention. Vision Research, 42, 1395-1401. [PubMed]

Tolias, A. S., Keliris, G. A., Smirnakis, S. M., \& Logothetis, N. K. (2005). Neurons in macaque area V4 acquire directional tuning after adaptation to motion stimuli. Nature Neuroscience, 8, 591-593. [PubMed]

Treisman, A. M., \& Gelade, G. (1980). A featureintegration theory of attention. Cognitive Psychology, 12, 97-136. [PubMed]

Treue, S., \& Martínez-Trujillo, J. C. (1999). Feature-based attention influences motion processing gain in macaque visual cortex. Nature, 399, 575-579. [PubMed]

Treue, S., \& Maunsell, J. H. (1996). Attentional modulation of visual motion processing in cortical areas MT and MST. Nature, 382, 539-541. [PubMed]

Turatto, M., Mazza, V., Savazzi, S., \& Marzi, C. A. (2004). The role of the magnocellular and parvocellular systems in the redundant target effect. Experimental Brain Research, 158, 141-150. [PubMed]
Ungerleider, L., \& Mishkin, M. (1982). Two cortical visual systems. In D. Ingle, M. Goodale, \& R. Mansfield (Eds.), Analysis of visual behavior (pp. 49-86). MA: MIT Press.

Valdés-Sosa, M., Bobes, M., Rodríguez, V., \& Pinilla, T. (1998). Switching attention without shifting the spotlight: Object-based attentional modulation of brain potentials. Journal of Cognitive Neuroscience, 10, 137-151. [PubMed]

Valdés-Sosa, M., Cobo, A., \& Pinilla, T. (1998). Transparent motion and object-based attention. Cognition, 66, B13-B23. [PubMed]

Valdés-Sosa, M., Cobo, A., \& Pinilla, T. (2000). Attention to object files defined by transparent motion. Journal of Experimental Psychology: Human Perception and Performance, 26, 488-505. [PubMed]

Viviani, P., \& Aymoz, C. (2001). Colour, form, and movement are not perceived simultaneously. Vision Research, 41, 2909-2918. [PubMed]

Wandell, B. A., Poirson, A. B., Newsome, W. T., Baseler, H. A., Boynton, G. M., Huk, A., et al. (1999). Color signals in human motion-selective cortex. Neuron, 24, 901-909. [PubMed] [Article]

Weissman, D. H., Roberts, K. C., Visscher, K. M., \& Woldorff, M. G. (2005). The neural correlates of momentary lapses of attention. Program No. 770.11. 2005 Abstract Viewer/Itinerary Planner. Washington, DC: Society for Neuroscience, 2005. Online.

Womelsdorf, T., Fries, P., Mitra, P. P., \& Desimone, R. (2006). Gamma-band synchronization in visual cortex predicts speed of change detection. Nature, 439, 733-736. [PubMed]

Zeki, S., \& Bartels, A. (1998). The autonomy of the visual systems and the modularity of conscious vision. Philosophical Transactions of the Royal Society of London. Series B, Biological Sciences, 353, 1911-1914. [PubMed] 


\subsection{Anticipation of impending signals lowers decision crite- rion without affecting perceptual sensitivity}

The preceding two articles have demonstrated influences of attention on visual processing. While attention is the most prominent top-down mechanism influencing perception, credit must also be payed to other cognitive factors modulating the sensory processing. For instance, based on prior experience, we constantly and implicitly maintain an internal representation of the likelihood of impending events, which enables us to allocate processing resources at the right moment in time and to prepare appropriate actions. Accurately predicting upcoming events requires a representation of the probability that a signal is about to occur given it has not occurred so far. This conditional probability is termed the hazard rate.

Recently, two electrophysiological studies have demonstrated that visual processing is modulated according to the hazard rate. In area MT (Ghose and Maunsell, 2002), the strength of attentional effects was strongly correlated with the probability of immediate signal occurrence. In area LIP (Janssen and Shadlen, 2005), the pattern of single neuron activity directly reflected the time course of the hazard rate. Both of these studies demonstrate that the observer's state of anticipation can substantially modulate the firing rates of visual neurons.

In addition, behavioral studies have shown that RT is inversely related to the time between a warning and a response-demanding stimulus, but only if the hazard rate increases (Klemmer, 1956; Requin and Granjon, 1969). However, the mechanism mediating this behavioral benefit has not been investigated so far. On the one hand, observers could sharpen their perceptual sensitivity based on the probability of an upcoming event, leading to improved sensory thresholds and faster RTs. On the other hand, subjects could also achieve faster RTs without sensory improvements, by changing their decision criterion according to the hazard rate.

In this study, we examined whether the behavioral benefits of signal anticipation are mediated by improvements in perceptual sensitivity or rather by adjustments in the internal response criterion. Using a combination of signal detection theory and behavioral reverse correlation we find that human observers adjust their internal decision criterion according to the probability of an upcoming event, while maintaining a constant perceptual sensitivity. 


\title{
Anticipation of Impending Signals Lowers Decision Criterion Without Affecting Perceptual Sensitivity
}

\author{
Steffen Katzner ${ }^{1,2}$, Laura Busse ${ }^{1,2}, \&$ Stefan Treue ${ }^{1,2,3}$ \\ ${ }^{1}$ Cognitive Neuroscience Laboratory, German Primate Center, Göttingen, Germany \\ 2 Bernstein Center for Computational Neuroscience, Göttingen, Germany \\ 3 Department of Biology, Georg-Elias-Müller Institute for Psychology, University of Göttingen, Germany
}

\begin{abstract}
An accurate prediction of upcoming events is crucial for the effective allocation of processing resources and for planning actions. Numerous behavioral studies have shown that reaction time is inversely related to the hazard rate, i.e. the conditional probability that a response-demanding signal is about to occur, given it has not occurred so far. Here, we used a combination of signal detection theory and a novel behavioral reverse correlation approach to investigate whether benefits of event predictability are mediated by improving perceptual sensitivity or by adjustments in decision-related variables over time. The data show that perceptual sensitivity $\left(d^{\prime}\right)$ is not affected by the hazard rate and does not change during a trial. In contrast, we observed a strong dependence of the decision criterion $(\beta)$ on the hazard rate. These results indicate that the human visual system actively tracks the probability of upcoming events by adjusting its internal decision criterion while maintaining a constant perceptual sensitivity.
\end{abstract}

\section{Introduction}

In the Seoul 1988 Olympics, the German decathlet Jürgen Hingsen, favorite for the gold medal, was disqualified for false-starting three times in the $100 \mathrm{~m}$ dash competition. Taking the enormous risk of being expelled from the Games can only be justified if performance is greatly enhanced by correctly anticipating the starter's gun. In fact, several laboratory experiments have demonstrated that human reaction time (RT) is faster if subjects can anticipate the point in time that a response demanding signal is likely to appear, compared to situations where such anticipation is impossible (Klemmer, 1956; Requin and Granjon, 1969). To correctly anticipate upcoming signals, an observers' brain must represent the conditional probability 
that a signal is about to appear, given it has not appeared yet. The resulting quantity is termed the hazard rate. Electrophysiological studies in non-human primates have recently revealed a positive correlation between the hazard rate and neuronal activity in two areas of visual cortex (medial temporal area (MT), Ghose and Maunsell (2002); lateral intraparietal area (LIP), Janssen and Shadlen (2005)). Given that these areas are specialized for sensory processing (MT), or represent an interface between sensory processing and motor planning (LIP), it seems possible that the hazard rate might influence sensitivity of visual processing. Here, we devised a behavioral paradigm to address the question whether the correct prediction of upcoming signals can lead to changes in sensory sensitivity or mainly causes adjustments in decision-related variables, hence coming secondary to sensory processing.

To investigate the benefits of correctly anticipating upcoming signals we used a seveninterval visual-motion signal detection paradigm, which allowed us to disentangle changes in perceptual sensitivity from changes in the decision criterion (Green and Swets, 1966). Subjects had to detect a single, threshold-level coherent motion signal (target) among consecutive, discrete presentations of brief random-motion dot stimuli (RDPs), separated by blank intervals (Fig. 1a). They were instructed to report the target as soon as they detected it. Predictability of an upcoming target was manipulated by using two different hazard conditions (Fig. 1b). In one block, the hazard rate was increasing from interval to interval such that the likelihood of future events increased with every interval passing by. In a second block, the hazard rate remained constant, and therefore the likelihood of instant target signal appearance did not vary with the number of past intervals. The discrete, seven-interval design we used offers the advantage to evaluate hits (i.e., correct positive responses) and false alarms (i.e., incorrect positive responses) for every single interval in the stimulus sequence. Adopting the framework of signal detection theory, we used these hits and false alarms to compute measures of perceptual sensitivity $\left(d^{\prime}\right)$ and decision criterion $(\beta)$ as a function of stimulus interval and hazard condition.

Moreover, assuming that a subject's response results from some perceptual filter being applied to the motion signal in the stimulus, we performed a noise-based estimation of this filter, or kernel, and examined how it changed from interval to interval, separately for each hazard condition. In this approach, the underlying idea is to characterize the linear relationship between certain stimulus properties and subsequent behavioral responses, from which mechanisms of sensory processing, i.e., perceptual filters, can then be inferred. In this context, changes in the overall shape of the perceptual filter would indicate changes in perceptual sensitivity. In contrast, multiplicative scaling of the filter, without significant changes in its overall shape, would be consistent with changes in the decision criterion (Eckstein et al., 2002). For this approach, we adapted the reverse correlation technique that has been used in neurophysiological studies (Cook and Maunsell, 2004).

The signal detection analysis showed that perceptual sensitivity remains unchanged from interval to interval, independent of the hazard condition. In contrast, the decision criterion is strongly lowered from interval to interval, but only if the hazard rate is increasing. This clearly indicates that the behavioral benefit of correctly anticipating relevant signals is mediated by 
A

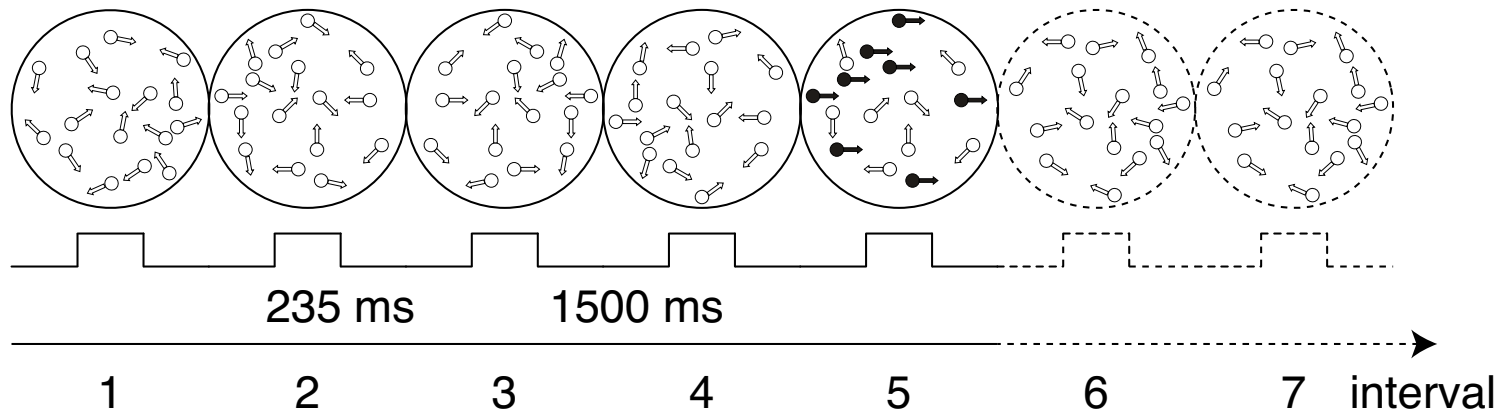

B Uniform

Geometric
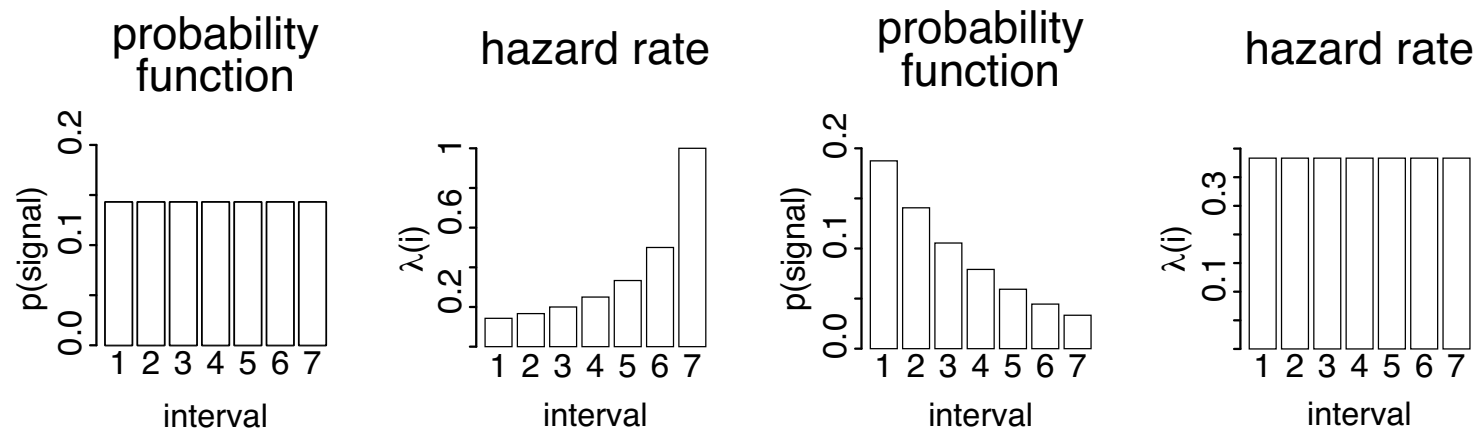

Figure 1: Methods. (a) Sequence of events in a single trial. Subjects viewed a temporal sequence of 1-7 brief presentations of a centrally displayed, moving random dot pattern, followed by a blank screen. The subject's task was to press a button upon detection of a threshold-level coherent motion signal of a pre-defined direction, embedded in a sequence of otherwise random noise stimuli ( $0 \%$ coherence). Trials were terminated after the response (hit or false alarm), but also if the target had been presented and no response was given during the following interstimulus-interval (miss). Subjects received auditory feedback after each trial. For illustrative purposes, the target signal in the figure consists of rightward motion, and was presented in the fifth interval (signal and noise dots were indistinguishable in the actual experiment). Dotted lines indicate parts of the trial sequence that were not actually shown since the trial had already been terminated. (b) Target-interval distributions and corresponding hazard rates in the two different hazard conditions. In different blocks of trials, the stimulus interval containing the threshold-level coherent motion signal was drawn from a uniform or geometric distribution, respectively. While the uniform probability distribution yields an increasing hazard function, the hazard function of the geometric probability distribution remains constant across intervals. Each subject performed 9 runs containing 53 trials under each of the two hazard condition, with the order of conditions being counterbalanced across subjects. The subjects were neither informed about the different signal interval distributions nor about the change in the hazard conditions. 
changes in the decision criterion and not by changes in perceptual sensitivity. Consistent with this finding, our behavioral reverse correlation revealed that the perceptual filters did not qualitatively change their shape from interval to interval. Furthermore, an interval-dependent multiplicative scaling of the filter tuning seemed evident, and this scaling was more reliable for the increasing than for the constant hazard rate. Taken together, these results indicate that the human visual system dynamically updates the probability of upcoming events by adjusting its internal decision criterion while maintaining a constant perceptual sensitivity.

\section{Results}

\section{Signal Detection Analysis}

We obtained measures of perceptual sensitivity and response criterion for each signal interval and hazard condition using Signal Detection Theory (Green and Swets, 1966) (see Methods). The results are summarized in Fig. 2. In both hazard conditions, perceptual sensitivity $\left(d^{\prime}\right)$ remained unchanged across intervals of stimulus presentation (ANOVA, $P=0.18$ ), and there was no difference in the overall pattern of $d^{\prime}$ values between the two hazard conditions (ANOVA, $P=0.38$ ). In contrast, the response criterion $(\beta)$ strongly varied between stimulus intervals, and this variation depended on which hazard condition was used (ANOVA, $P<$ 0.002). While $\beta$ remained unaffected for the constant hazard rate (ANOVA, $P=0.8$ ), it strongly decreased across intervals for the increasing hazard rate (ANOVA, $P=0.003$, linear trend analysis, $P<0.0001)$. For the first intervals, changes in $\beta$ were more pronounced before leveling off towards the end of the sequence (quadratic trend analysis, $P=0.04$ ).

\section{Reverse correlation analysis}

Next, we performed a noise-based reverse correlation analysis to estimate the linear filter which, applied to the motion stimulus, best characterizes the subjects' responses for each hazard condition and interval. For this analysis, we only used stimuli containing purely random motion, i.e., stimuli that subjects incorrectly responded to ('false alarms'), or stimuli that subjects correctly did not respond to ("correct rejections"). Due to the random placement and motion direction of dots, the effective motion signal in such stimuli varies from trial to trial. To capture the effective motion signal in each noise stimulus we first computed, from frame to frame, every possible motion vector along which every single dot could have moved (Barlow and Tripathy, 1997; Cook and Maunsell, 2004). Second, we constructed a histogram of the obtained motion vectors, ranging from 0 to $360^{\circ}$, with a bin size of $30^{\circ}$. In order to reveal the relationship between stimuli of varying effective motion signals and subsequent behavioral responses, we performed a logistic multiple regression analysis to predict, on the basis of the binned effective motion signals in the random noise stimuli, false alarms and correct rejections, 

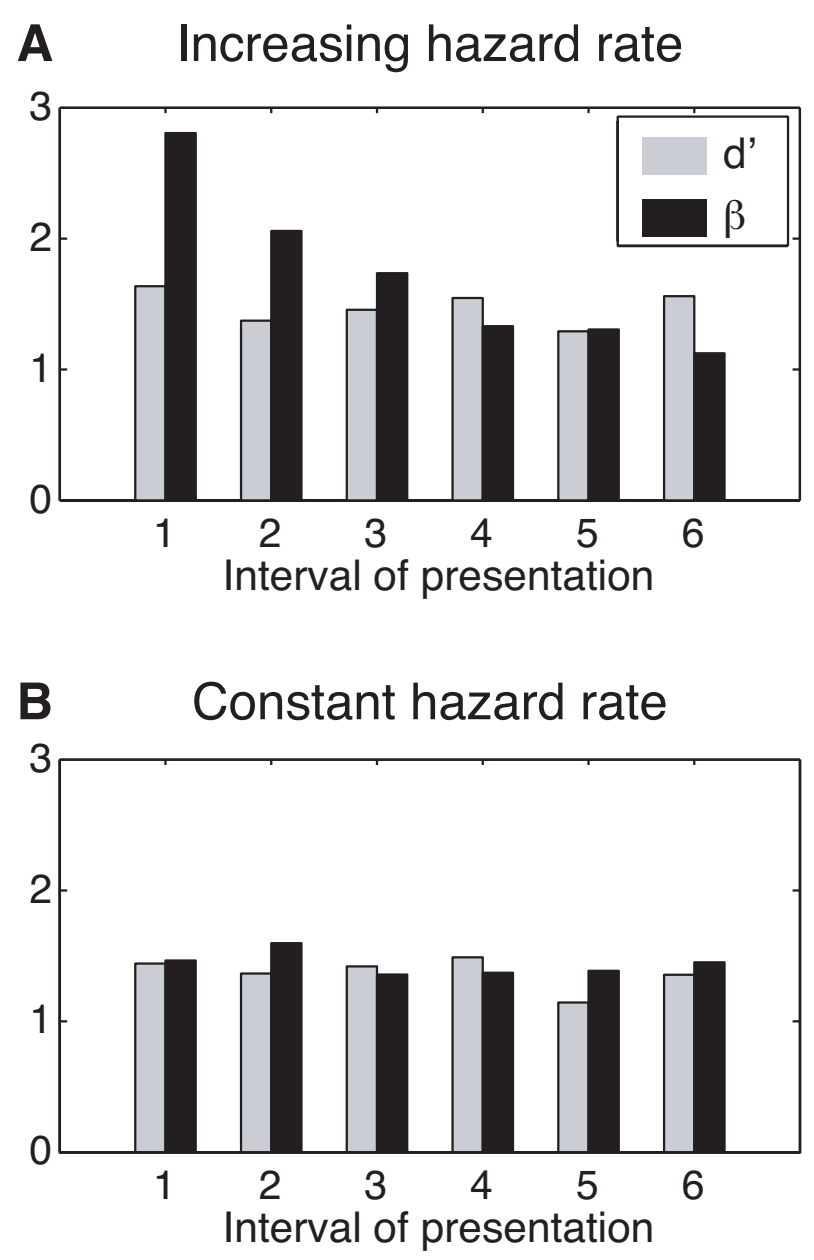

Figure 2: Results from signal detection analysis. Separately for the increasing (a) and constant (b) hazard condition, we calculated perceptual sensitivity $\left(d^{\prime}\right)$ and response criterion $(\beta)$ for each stimulus interval. Perceptual sensitivity is not influenced by the hazard condition, nor does it vary across intervals. In contrast, the response criterion strongly decreases across intervals, but only in the condition with increasing hazard rate. The decrease is most pronounced in the first four intervals. These results clearly show that benefits of signal anticipation are mediated by changes in the response criterion rather than by changes in perceptual sensitivity. 


\begin{tabular}{lllll}
\hline \hline & \multicolumn{4}{c}{ Hazard rate } \\
\cline { 2 - 5 } & \multicolumn{2}{c}{ constant } & \multicolumn{2}{c}{ increasing } \\
\cline { 2 - 5 } & \multicolumn{1}{c}{$\mathrm{n}$} & \multicolumn{1}{c}{$\mathrm{n}$} & $\mathrm{p}$ \\
\cline { 2 - 5 } Interval 1 & 2112 & $1.11 * 10^{-16}$ & 2417 & $6.16 * 10^{-6}$ \\
Interval 2 & 1262 & $1.08 * 10^{-8}$ & 1919 & $4.44 * 10^{-16}$ \\
Interval 3 & 778 & $4.68 * 10^{-10}$ & 1353 & $5.87 * 10^{-8}$ \\
Interval 4 & 458 & $3.24 * 10^{-14}$ & 842 & $5.57 * 10^{-11}$ \\
\hline \hline
\end{tabular}

Table 1: Summary of logistic regression models. A penalized maximum-likelihood algorithm (Firth, 1993) was used to fit a multiple logistic regression model to the subjects' behavioral responses (correct rejections or false alarms) triggered by random variations in the motion components of the preceding noise stimulus. While the number of observations $(n)$ necessarily decrease with increasing intervals, the model provides a very good description for the first four intervals ( $p$-values indicate the significance of the regression coefficients as determined by a Likelihood ratio test).

respectively. The resulting regression coefficients can be expressed as linear filter weights, and we estimated these weights separately for each interval in each hazard condition. In multiple regression, reliable estimates of the regression coefficients generally require a sufficient number of observations. Since the number of false alarms and correct rejections necessarily decreases towards the end of our stimulus sequence, we restricted the regression analysis to the first four intervals, combining the data from all subjects. Note also that decreases in $\beta$ are most pronounced for the first four intervals only (see Fig. 2). Table 1 summarizes the number of observations together with the validity of the regression models for the first four intervals in both hazard conditions.

Fig. 3a shows the kernel weights as estimated by the logistic regression analysis, after they have been smoothed with a Gaussian filter (width of 1 bin). If benefits of target signal anticipation are mediated by adjustments in sensory processing, we should observe pronounced changes in the kernels' overall shape from interval to interval. For instance, improved sensory performance could be achieved by increasing the weights for the target direction while decreasing the weights for other directions. In contrast, if benefits of target signal anticipation are mainly due to non-sensory, decision-related adjustments, we should observe multiplicative scaling only, without major changes in the kernel's shape, i.e., an increase of the weights for all directions by a constant factor. As can be seen in Fig. 3a, the general shape of the kernels does not change much from interval to interval, and this holds true for both hazard conditions. To evaluate changes in shape statistically, we scaled the kernel in each interval by a constant factor, giving the best fit to the kernel in the subsequent interval. With a true multiplicative scaling, the kernel for a given interval should be perfectly predictable by scaling the preceding kernel with the corresponding factor. However, because of noise in the estimation process this will never be the case, but the variance in the residuals can be used to assess the goodness of 


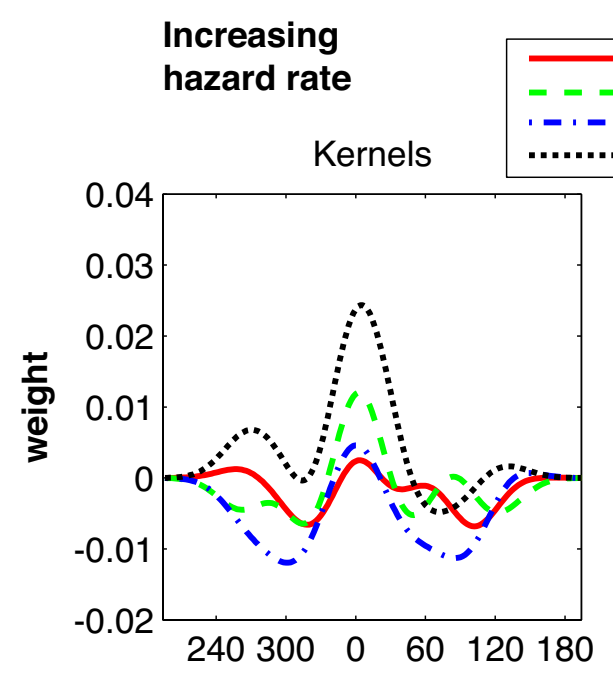

\section{Constant hazard rate}

Kernels
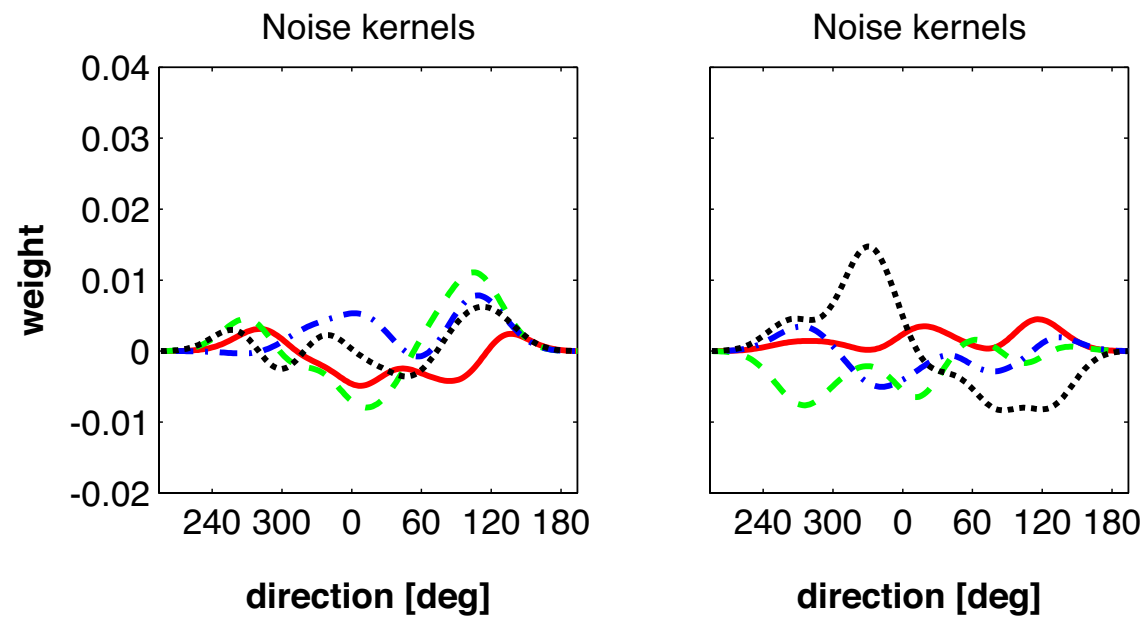

Figure 3: Results from behavioral reverse correlation analysis. The top panels show the kernels estimated by combining data from 7 subjects for the increasing (left) and constant (right) hazard condition, the bottom panel the corresponding noise kernels computed forward in time. In each condition, the signal direction $\left(0^{\circ}\right)$ closely corresponds to the point with highest amplitude. Differences between kernels in successive intervals can be attributed to multiplicative scaling without significant changes in kernel shape (but see discussion). Kernel amplitudes, i.e. the differences between maximal and minimal kernel weight, generally increase from interval to interval in the increasing hazard condition, which is in accord with decreases in the response criterion. Remarkably, the kernel for the first interval is almost flat in the increasing hazard condition, while it is much more pronounced in the constant hazard condition. This is in close agreement with the strong difference in response criteria for this interval between the two hazard conditions. 
the match between the actual and the predicted kernel. If the residual variance is much more pronounced relative to the variance expected by chance, this would reject the hypothesis of multiplicative scaling. To estimate residual variance obtained by chance, we computed 'noise kernels' by correlating false alarms and correct rejections with stimuli that have never been shown, or have only been shown in future trials, relative to the current behavioral response. Such noise kernels can be seen in Fig. 3b. Theoretically, these noise kernels should be flat since motion signals in the stimuli entering the prediction equations cannot be related to the current behavioral response. Therefore, profiles of noise kernels deviating from a flat line reflect the noise inherent in our kernel estimation process. Apparently, most of the noise kernels have a non-flat shape and it is therefore not surprising, that none of the statistical tests for deviations from multiplicative scaling reached significance ( $p>0.90$ for all comparisons). Hence, this finding has to be interpreted in light of the enormous variance that is present in the noise kernels (see discussion).

We also examined whether any interval-dependent scaling of the kernels was more pronounced for the constant than for the increasing hazard rate. As a measure of the strength of tuning for these kernels we computed the peak-to-peak amplitude, separately for each interval and hazard condition. Using a bootstrap-based analysis (Efron and Tibshirani, 1993), we found a significant positive increase in peak-to-peak amplitude as a function of interval for the increasing hazard rate $(P=0.03)$. While the peak-to-peak amplitudes for the constant hazard rate also tend to increase, this effect closely failed to reach statistical significance $(P=0.078)$. To validate that the increase in amplitudes is not caused mainly by an increase in noise, we calculated the signal-to-noise ratio, defined as the variance of the kernel divided by the variance of the corresponding noise kernel for each interval. Interestingly, for the increasing hazard rate, the signal-to-noise ratio increases across intervals, while it decreases only in the constant hazard rate condition. Taken together, our noise-based reverse-correlation analysis revealed kernels, or perceptual tuning curves, that expressed a distinct peak and a Mexican-hat shaped profile. Evidently, the profiles of these kernels seem to reflect changes in the hazard rate. While the kernel in the first interval is almost flat for the increasing hazard rate, the corresponding kernel for the constant hazard rate is much more pronounced in the corresponding first interval. Moreover, the scaling of the amplitudes seems to be more obvious for the increasing than for the constant hazard rate. However, because of the noise inherent in our kernel estimation procedure we are unable to draw firm conclusions as to whether the scaling is truly multiplicative or not.

\section{Discussion}

In this experiment, we addressed the question whether benefits of signal anticipation are mediated by changes in perceptual sensitivity or by adjustments of decision-related variables.

We manipulated the predictability of a pre-defined threshold-level target signal in a sequence of noise stimuli with increasing and constant hazard rates, respectively. With an increasing 
hazard rate, the immediate appearance of the target signal becomes more and more likely, given it has not appeared yet. In contrast, with constant hazard rates the conditional probability of instantaneous target signal appearance remains constant. Using signal detection theory we find that the magnitude of predictability of an upcoming target signal strongly influences the response criterion $(\beta)$, while it leaves perceptual sensitivity $\left(d^{\prime}\right)$ unaffected. This shows that benefits of successful signal anticipation are mediated by adjustments in decisionrelated variables, rather than changes in sensory processing. From reaction time research it has long been known that RT strongly depends on the variability and distribution of the 'fore-period', i.e., the time elapsing between the presentation of a warning stimulus and a response demanding stimulus (Klemmer, 1956; Drazin, 1961; Bertelson and Tisseyre, 1968; Requin and Granjon, 1969; Nickerson and Burnham, 1969; Niemi and Näätänen, 1981). RTs considerably decrease with longer fore-periods, but only if the immediate presentation of the response demanding signal becomes more and more likely over time. In contrast, RTs remain constant in the absence of such predictability. While this shows an influence of the magnitude of predictability of upcoming signals on behavior, such a measure cannot distinguish between sensory versus decision-related adjustments in stimulus processing. Unlike previous work, our discrete-interval signal detection approach offers the advantage to disentangle these alternatives, shedding light on the mechanism involved in target signal anticipation.

Various electrophysiological studies in non-human primates have investigated the neuronal basis of decision making. In particular, neurons in the lateral intra-parietal (LIP) cortex have been shown to play a major role in decision processes that guide behavior (Hanks et al., 2006; Platt and Glimcher, 1999), and their activity is strongly modulated by the hazard rate (Janssen and Shadlen, 2005). Hence, hazard-rate dependent modulations in the activity of area LIP might represent the neuronal basis for the variations in response criterion we observed.

In addition to the signal detection analysis, we adapted the reverse correlation technique for an analysis of behavioral data to assess how estimates of perceptual filters are influenced by different hazard rates. We reverse correlated behavioral responses with preceding stimuli to estimate kernels describing the subjects' weighting of the motion signal as a function of hazard rate and time. Reverse correlation has first been used in electrophysiological studies to investigate receptive field structures of sensory neurons (Sakai et al., 1988; Ringach and Shapley, 2004), and has subsequently been adopted for characterizing human observer templates in psychophysical studies (for a review, see Neri and Levi, 2006). While appropriate methods, e.g. classification image techniques, are available for psychophysical reverse correlation approaches with stationary white-noise stimuli, application of these techniques to visual motion is not straightforward because of autocorrelations in the stimulus. Other work has evaded this problem by using an equivalent linear regression approach (Ahumada Jr. and Lovell, 1971; Cook and Maunsell, 2004; DiCarlo et al., 1998; Theunissen et al., 2001; Blake and Merzenich, 2002). However, in none of these studies the dependent variable consisted of binary outcomes. Here, we used a logistic multiple regression approach which allowed us to reverse correlate binary behavioral outcomes, (i.e., false alarms or correct rejections) with the motion signal in the stimulus preceding that response. The validity of our approach is strongly supported 
by the significance of the regression model fits (see Table 1). Since the logistic regression approach can be applied in virtually any detection task context, this method is a useful addition to previous implementations of psychophysical reverse correlation.

Reverse correlation critically depends on random variations in a noisy stimulus that are correlated with observed responses. We introduced noise into moving random dot patterns by randomly assigning different directions of motion and speeds to individual dots. While speed was always kept constant for all dots across all trials, it would be optimal to vary the motion direction of individual dots randomly for every single trial. However, our stimulus-generating software currently does not allow this. While the starting positions of individual dots vary across trials, the random direction assigned to each dot is constant throughout a block of trials. Consequently, the distribution of possible motion vectors varies much more strongly across blocks than across trials within a given block. This inability to randomly re-assign motion directions to individual dots on a trial-by-trial basis most likely reduces the quality of kernel estimates in the logistic regression approach, since the variance in the motion signal is artificially reduced. In extreme cases, different responses (false alarms or correct rejections, respectively) are correlated with stimuli containing identical motion vectors. This reduced variation in the motion signal could be one reason why we failed to compute statistically reliable kernels for each subject separately. As it stands, our reverse correlation analysis did not reveal any evidence for changes in the overall shape of perceptual filters across intervals, which would be consistent with the absence of changes in sensory processing. However, the fact that we do not find these changes is mainly due to the lack of statistical power and the noise inherent in our kernel estimation process. Currently, we are repeating the experiment using an improved version of the software that allows a random re-assignment of motion directions for every single trial. Note however, that despite our problems of incomplete randomization, we were still able to extract meaningful estimates of the subjects' kernels (Table 1), from which one can readily extract, e.g., the designated target signal direction (Fig. 3a,b).

In summary, we have devised a discrete-interval signal detection paradigm that allows us to disentangle alternative mechanisms explaining behavioral benefits of successful anticipation of upcoming signals. Applying methods of signal detection theory we demonstrate that behavioral benefits of signal anticipation are not mediated by changes in sensory processing but rather result from adjustments in decision-related variables. As a complementary approach, we have used behavioral reverse correlation analysis to estimate perceptual filters of human observers, and have examined whether predictability of upcoming signals can lead to changes in these filters. Although the results from these latter analyses tend to support the findings obtained with signal detection theory, we cannot make firm conclusions about the absence of significant changes in the perceptual filters yet.

\section{Methods}

Subjects. 8 subjects (ages 21-28, 2 males and 6 females) participated in this study. All had 
normal or corrected-to-normal vision. They gave informed written consent and were naïve as to the purpose of the experiment.

Stimulus and apparatus. The stimulus was composed of dots moving within a virtual circular aperture of $5^{\circ}$ in diameter. A total of 250 dots was presented within this aperture. Each dot subtended $0.075^{\circ}$ of visual angle. All the dots were black and the RDP was plotted at the center of gaze, against a white background. All dots moved at a speed of $5^{\circ} / \mathrm{s}$. A noise stimulus was characterized by all dots moving in random directions ( $0 \%$ coherence). In contrast, a target motion signal exhibited some threshold level of coherent motion, which was embedded in the noise. The stimulus was presented on a VGA monitor (Lacie, Electron22 Blue IV) operating at a refresh rate of $85 \mathrm{~Hz}$ and a resolution of 80 pixels per degree of visual angle. Presentation of the stimulus and recording of the responses was controlled by an Apple Power Mac G4 computer.

Design and procedure. At trial start, a stationary dot pattern of $235 \mathrm{~ms}$ duration was presented as a warning signal. Following this, subjects viewed a temporal sequence of $1-7$ brief presentations of a centrally displayed, moving random dot pattern $(235 \mathrm{~ms})$, always followed by a blank screen (1500 ms). The subject's task was to press a button upon detection of a threshold-level coherent motion signal of a pre-defined direction, embedded in a sequence of otherwise random noise stimuli ( $0 \%$ coherence). For half of the subjects, the target signal consisted of rightward motion, for the other half leftward motion was used. Trials were terminated after a subject's response (hit or false alarm), but also after the target had been presented and no response was given before the next stimulus in the sequence would have been presented (miss). Subjects received auditory feedback after each trial. An increasing hazard rate was realized by drawing the target-interval for a given trial from a uniform distribution defined over the values $1-7$. A constant hazard rate was achieved by drawing the target-interval from a geometric, i.e. non-aging, distribution $($ mean $=4)$. In case the draw from the geometric distribution produced a number larger than 7 , no target signal was presented and the trial was considered a no-go. The experiment was divided into 6 sessions, each consisting of 3 blocks, that were performed on consecutive days. For one half of the subjects, the hazard rate was kept constant for the first nine blocks, and remained increasing for the second nine blocks. For the second half of the subjects, this assignment was reversed. Subjects were neither informed about the different signal interval distributions nor about the change in hazard conditions. For each subject and before each single block, a standard two-interval forced-choice staircase algorithm (Kaernbach, 1991) was run to determine the individual, practice-dependent level of coherent motion signal that was required to achieve $75 \%$ correct responses. This current estimation of individual threshold-level motion coherence was then used for the subsequent experimental block. Such a single experimental block was composed of 53 trials. The first three trials were used by the subjects to get acquainted with the strength of the target signal and were not included in any of the analyses.

Data analysis. One subject was excluded from the analyses because he did not produce any false alarms in the sixth interval. In such a case, meaningful signal detection measures $\left(d^{\prime}\right.$ and $\beta$ ) cannot be computed. All analyses were performed using Matlab (The MathWorks, Inc.) 
and the R environment for statistical computing (R Development Core Team, 2006).

Signal detection analysis. We used signal detection theory (Green and Swets, 1966) to compute measures for perceptual sensitivity $\left(d^{\prime}\right)$ and response criterion $(\beta)$. These two measures were determined for every combination of subject, signal interval, and hazard condition. Statistical significance was evaluated with a two-way Analysis of Variance (ANOVA), separately for $d^{\prime}$ and $\beta$, involving the within-subject factors hazard condition (increasing vs. constant) and stimulus interval (1-6). Interval 7 was excluded from analysis since, in the case of an increasing hazard rate, no false alarms can be generated in this interval. The Greenhouse-Geyser correction has been applied where appropriate.

Reverse correlation analysis. Following Cook and Maunsell (2004), we assumed that an subject's response $(R)$ is the result of a perceptual filter $(K)$ applied to the motion signal in the stimulus $(M)$, which can be expressed as a convolution

$$
R=K * M
$$

The convolution in Equation 1 can also be formulated as a linear regression problem (Ahumada Jr. and Lovell, 1971). Since we regress motion components on binary response outcomes, i.e. false alarms (1) or correct rejections (0), we used multiple logistic regression to estimate the kernel weights. The logistic regression model is a special case of the general linear model, in which the link function consists of the $\operatorname{logit}$ transformation $\log [p /(1-p)]$, symbolized by $\operatorname{logit}(p)$ (Agresti, 1996). Positive values for estimated coefficients reflect an increased, negative coefficients a decreased probability for an outcome of 1 , respectively. Thus, our regression model is given by

$$
\operatorname{logit}\left(p_{i}\right)=\log \left(\frac{p_{i}}{1-p_{i}}\right)=c+\sum_{d=1}^{12} k_{i, d} m_{i, d}
$$

where $c$ corresponds to the intercept term, $i$ is an index for interval, $d$ an index for direction of motion, $k$ represents the kernel weights, and $m$ the strength of the direction signal in the random noise stimulus. Using a bin size of $30^{\circ}$, Equation 2 expands to

$$
\operatorname{logit}\left(p_{i}\right)=c+k_{i, 15} m_{i, 15}+k_{i, 45} m_{i, 45}+\ldots+k_{i, 345} m_{i, 345}
$$

The obtained kernel weights as determined by the regression analysis are independent. However, since adjacent kernel weights are correlated, we have smoothed the kernel weights with a Gaussian function having a sigma of 1 bin $\left(30^{\circ}\right)$. To compute kernels across subjects, we have horizontally flipped the kernels for those subjects who had been assigned leftward motion as the target direction.

To test for differences in shape between kernels in successive intervals we adapted the procedure proposed by Cook and Maunsell (2004). First, we determined a best-fit scaling factor by which the kernel weights for a given interval $(i)$ would have to be multiplied to 
obtain the corresponding kernel weights for the subsequent interval $(i+1)$. To do so, we minimized a $\chi^{2}$-merit function with respect to the scaling factor $\gamma$ (Press et al., 1992):

$$
\chi^{2}=\sum_{d=1}^{12} \frac{\left(\gamma k_{i_{d}}-k_{i+1_{d}}\right)^{2}}{\gamma^{2} \sigma_{\text {noise }_{i}}^{2}+\sigma_{\text {noise }_{i+1}}^{2}}
$$

In Equation 4, the denominator represents the weighted sum of variances for 'noise kernels' $\left(\sigma_{\text {noise }}^{2}\right)$ in two successive intervals $i$. We computed these 'noise kernels' by correlating behavioral responses with such 0\%-coherence stimuli that have never been presented (because the trial had ended before that particular stimulus was going to be shown; see, e.g., the last two stimuli in Fig. 1), or that were going to be presented in future trials, relative to the current behavioral response (Fig. 3 b).

Second, we predicted kernel weights for interval $i+1$ by multiplying the kernel for interval $i$ with the estimated scaling factor. Such predictions will never be perfect, though, and the resulting residual kernels $K_{\text {res }}$ are given by the difference between the predicted kernel weights and the actual kernel weights for interval $i+1$.

$$
K_{\text {res }}=\gamma K_{i}-K_{i+1}
$$

Non-zero weights in the residual kernel, however, not only reflect deviations from multiplicative scaling, but also arise because of noise inherent in the estimation procedure. To disentangle these two components, we predicted the variance of $K_{\text {res }}$ due to noise $\left(\sigma_{0}\right)$ by the weighted sum of the variances in pairs of successive noise kernels.

$$
\sigma_{0}=\gamma^{2} \sigma_{\text {noise }_{i}}^{2}+\sigma_{\text {noise }_{i+1}}^{2}
$$

where $\gamma$ is our estimated multiplicative scaling factor. Deviations from multiplicative scaling would result in a larger variance in the residual kernel $K_{\text {res }}$ compared to the variance predicted on the basis of noise kernels $\left(\sigma_{0}\right)$. Statistical significance can then be evaluated by

$$
L=\frac{v \sigma_{r e s}^{2}}{\sigma_{0}^{2}}
$$

where $L$ follows a $\chi^{2}$-distribution with $v=12-1$ degrees of freedom (Zar, 1999).

Finally, we used a bootstrap approach to test whether the peak-to-peak amplitudes increased as a function of interval for the two hazard conditions. We first determined, for each interval separately, the difference between the maximal and minimal kernel weights and computed the slope of the best-fit line through the amplitude values. To test whether the slope of this line is significantly different from zero, we created bootstrap replicates of kernels by sampling with replacement the weights for each motion direction randomly from different intervals. We then smoothed the bootstrap replicates of the kernels with a Gaussian filter (sigma $=1 \mathrm{bin}$ ), before determining the slopes of the best-fit line through the amplitude values. We repeated this procedure 10000 times to obtain a distribution of bootstrapped slopes, which in 
turn allowed us to determine statistical significance of the actual slope. This procedure was done separately for increasing and constant hazard conditions.

\section{References}

Agresti, A. (1996). An introduction to categorical data analysis. Wiley, New York.

Ahumada Jr., A. J. and Lovell, J. (1971). Stimulus features in signal detection. Journal of the Acoustical Society of America, 49(6):1751-1756.

Barlow, H. and Tripathy, S. P. (1997). Correspondence noise and signal pooling in the detection of coherent visual motion. Journal of Neuroscience, 17(20):7954-7966.

Bertelson, P. and Tisseyre, F. (1968). The time-course of preparation with regular and irregular foreperiods. Quarterly Journal of Experimental Psychology, 20(3):297-300.

Blake, D. T. and Merzenich, M. M. (2002). Changes of AI receptive fields with sound density. Journal of Neurophysiology, 88(6):3409-3420.

Cook, E. P. and Maunsell, J. H. (2004). Attentional modulation of motion integration of individual neurons in the middle temporal visual area. Journal of Neuroscience, 24(36):79647977.

DiCarlo, J. J., Johnson, K. O., and Hsiao, S. S. (1998). Structure of receptive fields in area 3b of primary somatosensory cortex in the alert monkey. Journal of Neuroscience, 18(7):26262645.

Drazin, D. H. (1961). Effects of foreperiod, foreperiod variability, and probability of stimulus occurrence on simple reaction time. Journal of Experimental Psychology, 62:43-50.

Eckstein, M. P., Shimozaki, S. S., and Abbey, C. K. (2002). The footprints of visual attention in the Posner cueing paradigm revealed by classification images. Journal of Vision, 2(1):2545.

Efron, B. and Tibshirani, R. (1993). An Introduction to the Bootstrap. Chapman and Hall, London.

Firth, D. (1993). Bias reduction of maximum likelihood estimates. Biometrika, 80(1):27-38.

Ghose, G. M. and Maunsell, J. H. (2002). Attentional modulation in visual cortex depends on task timing. Nature, 419(6907):616-620.

Green, D. M. and Swets, J. A. (1966). Signal detection theory and psychophysics. Wiley, New York. 
Hanks, T. D., Ditterich, J., and Shadlen, M. N. (2006). Microstimulation of macaque area LIP affects decision-making in a motion discrimination task. Nature Neuroscience, 9(5):682-689.

Janssen, P. and Shadlen, M. N. (2005). A representation of the hazard rate of elapsed time in macaque area LIP. Nature Neuroscience, 8(2):234-241.

Kaernbach, C. (1991). Simple adaptive testing with the weighted up-down method. Perception \&5 Psychophysics, 49(3):227-229.

Klemmer, E. T. (1956). Time uncertainty in simple reaction time. Journal of Experimental Psychology, 51(3):179-184.

Neri, P. and Levi, D. M. (2006). Receptive versus perceptive fields from the reverse-correlation viewpoint. Vision Research, 46(16):2465-2474.

Nickerson, R. S. and Burnham, D. W. (1969). Response times with nonaging foreperiods. Journal of Experimental Psychology, 79(3):452-457.

Niemi, P. and Näätänen, R. (1981). Foreperiod and simple reaction time. Psychological Bulletin, 89(1):133-162.

Platt, M. L. and Glimcher, P. W. (1999). Neural correlates of decision variables in parietal cortex. Nature, 400(6741):233-238.

Press, W. H., Teukolsky, S. A., Vetterling, W. T., and Flannery, B. P. (1992). Numerical Recipes in C: the Art of Scientific Computing. Cambridge University Press, Cambridge, UK, 2nd edition.

R Development Core Team (2006). R: A Language and Environment for Statistical Computing. R Foundation for Statistical Computing, Vienna, Austria. ISBN 3-900051-07-0.

Requin, J. and Granjon, M. (1969). The effect of conditional probability of the response signal on the simple reaction time. Acta Psychologica, 31(2):129-144.

Ringach, D. and Shapley, R. (2004). Reverse correlation in neurophysiology. Cognitive Science, 28:147-166.

Sakai, H. M., Naka, K., and Korenberg, M. J. (1988). White-noise analysis in visual neuroscience. Visual Neuroscience, 1(3):287-296.

Theunissen, F. E., David, S. V., Singh, N. C., Hsu, A., Vinje, W. E., and Gallant, J. L. (2001). Estimating spatio-temporal receptive fields of auditory and visual neurons from their responses to natural stimuli. Network, 12(3):289-316.

Zar, J. H. (1999). Biostatistical Analysis. Prentice Hall, Upper Saddle River, NJ, 4th edition. 


\subsection{Spatial and feature-based effects of exogenous cueing on visual motion processing}

In the preceding studies, visual attention was directed to a stimulus for a rather long period of time, in some cases as long as several seconds. Many situations in everyday life, however, require that the focus of attention is shifted rapidly. The remaining two contributions address the dynamics of shifting visual attention.

Attention can be oriented in two fundamentally different ways. On the one hand, the attentional focus can be voluntarily directed to behaviorally relevant aspects in the visual scene. This mechanism has been termed endogenous attentional control, and has been described as effortful and relatively slow. On the other hand, attention can also be attracted reflexively to salient information in the visual field. Such exogenous shifts of attention are faster and occur in an automatic way.

In the laboratory, mechanisms of exogenous attentional orienting have been studied extensively using cueing paradigms. Here, a salient stimulus is briefly flashed at the potential location of an upcoming target. Even if such an exogenous cue does not reliably indicate the location of the upcoming target, subjects respond faster to targets at the cued compared to the uncued location, presumably owing to a reflexive shift of attention towards the source of stimulation (Posner and Cohen, 1984). Importantly, this RT benefit occurs only for targets presented in a short time interval after the cue. In contrast, with longer onset times ( $\sim 300 \mathrm{~ms})$ between cue and target, this facilitation of RTs vanishes and even turns into a slowdown. This phenomenon has been termed Inhibition of Return (IOR). It has been proposed that IOR plays an important role in visual foraging or other search behaviors in the sense that it encourages orienting towards novel locations by preventing attention from permanently focusing onto or revisiting the most salient stimulus.

While exogenous cueing is very well investigated for spatial shifts of attention, we have examined whether attention can also be automatically attracted to non-spatial stimulus features. We find that exogenous cueing only facilitates the cued feature at the cued location with short onset times between cue and target. In contrast, the later IOR is purely spatial in the sense that RTs are slower for the cued location, irrespective of the feature presented there. 


\title{
Spatial and feature-based effects of exogenous cueing on visual motion processing
}

\author{
Laura Busse $^{\mathrm{a}}$, Steffen Katzner ${ }^{\mathrm{a}}$, Stefan Treue ${ }^{\mathrm{a}, \mathrm{b}, *}$ \\ ${ }^{a}$ Cognitive Neuroscience Laboratory, German Primate Center, Kellnerweg 4, 37077 Göttingen, Germany \\ ${ }^{\mathrm{b}}$ Department of Biology, Georg-Elias-Müller Institute for Psychology, Göttingen University, Goßlerstrasse 14, 37073 Göttingen, Germany
}

Received 19 December 2003; received in revised form 29 April 2005

\begin{abstract}
In two experiments, we investigated the effects of exogenous cueing on visual motion processing. The first experiment shows that the typical pattern of reaction time (RT) effects, namely early facilitation and later inhibition of return (IOR), can be obtained using a color change as exogenous cue and a direction change as target. In the second experiment, we manipulated the validity of the cue independently with respect to location and feature using transparent motion stimuli. Facilitation of RTs with short cue-target interstimulus-intervals (ISIs) was only evident for targets with both the valid location and the valid feature. Furthermore, at longer cue-target intervals, RTs were prolonged for targets at the cued location, irrespective of the cued feature. These results demonstrate spatial and feature-based components of early facilitation and purely spatial IOR.
\end{abstract}

(C) 2006 Elsevier Ltd. All rights reserved.

Keywords: Visual attention; Reaction time; Motion transparency; Human

\section{Introduction}

Visual attention is the mechanism that allows us to choose behaviorally relevant information from the immense amount of input that impinges on our eyes. The flexible allocation of attention to spatial locations (e.g., Posner, 1980), different sensory features (like one particular color or motion direction $)^{1}$ (e.g., Sàenz et al., 2003), and objects (e.g., Blaser, Pylyshyn, \& Holcombe, 2000) is the central ability of our visual system to dynamically react to changing aspects of our environment and to varying behavioral goals.

In vision, cueing studies have provided insight in the dynamics of location-based, feature-based, and object-based attentional shifts. In general, such studies can be distin-

\footnotetext{
* Corresponding author. Fax: +49 5513851452.

E-mail address: treue@gwdg.de (S. Treue).

1 'Feature' refers to a particular property within a stimulus dimension, e.g., upwards motion is a feature within the stimulus dimension of motion, and red is a feature within the stimulus dimension of color (cf. Sàenz, Burac̊as, \& Boynton, 2003).
}

guished based on the nature of the cue: symbolic cues (arrows, etc.) are used for endogenous (or voluntary) orienting while peripheral cues (flashing stimuli, etc.) activate exogenous (or automatic) orienting processes (Posner \& Cohen, 1984). In the case of exogenous cueing, subjects generally react faster and more accurately after valid cues, but only for short intervals between the cue and target. If the cue-target interstimulus interval (ISI) exceeds around $300 \mathrm{~ms}$, reaction times will be slower (Posner \& Cohen, 1984), and responses less accurate (Handy, Jha, \& Mangun, 1999) for targets at the cued location than for targets at the uncued location. This latter effect of a peripheral cue has been termed Inhibition of Return (IOR) (see Klein, 2000 for a recent review). It has been suggested that IOR plays an important role in visual foraging behavior in that IOR prevents attention from permanently focussing onto or revisiting the most salient stimulus (Klein, 1988; Itti \& Koch, 2000).

The effects of exogenous cueing have not only been investigated in the spatial domain, but also with respect to feature-based and object-based attentional processes. Tipper, Driver, and Weaver $(1991,1994)$ were first to show 
object-based IOR. To dissociate location-based and objectbased IOR, they cued a moving object and found that, at long cue-target ISIs, responses were not inhibited for the initially cued location but inhibition moved with the cued object to its new location. Furthermore, with stationary stimuli, object-based and location-based inhibitory effects have been shown to combine in an additive fashion (Leek, Reppa, \& Tipper, 2003). However, other groups have failed to find independent location-based and object-based mechanisms of IOR (Christ, McCrae, \& Abrams, 2002; McAuliffe, Pratt, \& O'Donnell, 2001).

To investigate feature-based effects of IOR, typically a nonspatial stimulus attribute, such as color (Kwak \& Egeth, 1992; Law, Pratt, \& Abrams, 1995) or shape (Riggio, Patteri, \& Umilta, 2004), is repeated (valid condition) vs. non-repeated (invalid condition) for cue and target. The results obtained in these studies are mixed, in that some groups found feature-based inhibitory effects (Law et al., 1995; Riggio et al., 2004), whereas others did not (Kwak \& Egeth, 1992). However, in most of the studies, spatial and nonspatial attributes of the stimuli have not been manipulated independently (e.g., cues and targets were always presented at fixation (Kwak \& Egeth, 1992; Law et al., 1995)), thereby confounding spatial and feature-based effects of IOR. In addition, repetition of stimulus features might lead to adaptation (at least when presented at the same location) (e.g., Clifford, 2002; Muller, Metha, Krauskopf, \& Lennie, 1999) or repetition blindness (Fox \& de Fockert, 2001; Kanwisher, 1987; Taylor \& Klein, 1998).

Here, we investigate spatial and feature-based effects of exogenous cueing on visual motion processing using an experimental design that allows the independent manipulation of spatial and nonspatial stimulus attributes. In the first experiment, we show that spatial IOR can be obtained using a cue-target combination of stimulus attributes that are processed in two different visual pathways (Felleman \& Van Essen, 1991; Ungerleider \& Mishkin, 1982), namely a color change (ventral pathway) serving as the cue and a change in motion direction (dorsal pathway) serving as the target. In the second experiment, we independently vary the validity of the cue with respect to location and feature in order to disentangle spatial and feature-based effects of exogenous cueing. With short cue-target ISIs, we find facilitation of RTs only when the cue is valid with respect to both location and feature. Additionally, in conditions with longer cue-target ISIs, we obtain IOR for the cued location, irrespective of the previously cued feature. These results demonstrate location- and feature-based components of exogenous shifts of attention.

\section{Methods}

\subsection{Experiment 1}

Ten naive subjects (age 20-28, 6 female, 4 male) with normal or corrected-to-normal vision participated in the experiment. All subjects gave informed written consent and were paid for taking part in an one hour session in which they completed 5 blocks of 100 trials each. One subject was excluded from the data analysis since his performance was more than three standard deviations below the sample mean.

The experiment was conducted in a dimly illuminated and quiet room. Stimuli were presented on a VGA monitor (Quatographic, Color Station Professional) operated at a refresh rate of $85 \mathrm{~Hz}$ and a spatial resolution of 40 pixels/deg. Stimulus presentation and recording of responses was controlled by custom-made software running on an Apple Power Mac G4. Subjects placed their head on a chin-rest or a bite-bar positioned $57 \mathrm{~cm}$ from the monitor. During the trials, eye-movements were monitored using an infrared eyetracking system (ISCAN ETL-200). In case eye position deviated more than $1.5 \mathrm{deg}$ from a central fixation point the experiment was paused by the experimenter and subjects were re-instructed to maintain fixation.

The stimulus was composed of two circular apertures (radius $1.8 \mathrm{deg}$ ) of moving dots (dot density: 8 pixels $/ \mathrm{deg}^{2}$ ) centered $5 \mathrm{deg}$ to the left and the right of the fixation point. Dots were gray $\left(12 \mathrm{~cd} / \mathrm{m}^{2}\right)$ on a black background, subtending $0.05 \mathrm{deg}$ of visual angle in width. The dots in each aperture coherently moved at a speed of $7 \mathrm{deg} / \mathrm{s}$, in one of 4 possible directions $(45,135,225$, and $315 \mathrm{deg}$ deviation from vertical). In each trial, the directions of the two dot patterns differed by at least $90 \mathrm{deg}$. During the course of the trial, some dots changed their color to red. For each participant separately, flicker fusion photometry was conducted to achieve sub-

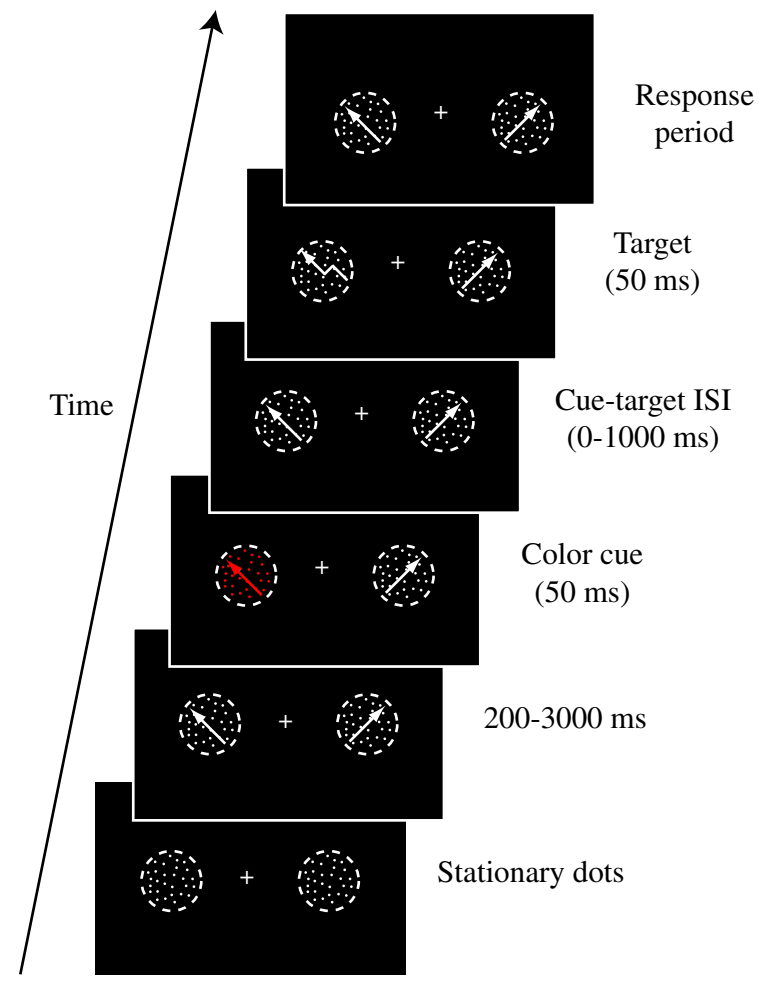

Fig. 1. Schematic trial structure for Experiment 1. Two random dot patterns were presented to the left and right of fixation. After trial start, the dots in the apertures moved coherently in directions differing by at least 90 deg for $200-3000 \mathrm{~ms}$. Randomly during this time period a cue consisting of a color change to red ( $50 \mathrm{~ms}$ duration) appeared in one of the apertures. This cue was followed by a target defined as a brief change in the direction of motion (except for a 'catch trial' condition, in which no target was presented, see below). The subjects' task was to respond as quickly as possible to the direction change (or to withhold the response in case of a 'catch trial'). The example illustrates a valid trial since the target direction change occurs in the previously cued motion pattern. Note that the dashed outlines of the apertures as well as the arrows symbolizing the movement of the dots were not present in the actual experiment. (For interpretation of the references to color in this figure legend, the reader is referred to the web version of this paper.) 
jective isoluminance for the gray and red colors. While the intensity of the gray was held constant for all subjects, the intensity of the red gun was adjusted by each subject until minimal flicker was perceived at a flicker rate of $16 \mathrm{~Hz}$. The adjusted intensity of the red gun was averaged across 25 trials and the result was used in Experiment 1.

Fig. 1A shows a schematic layout of the trial structure. At the beginning of each trial, stationary dots were presented in the apertures. When subjects initiated the trial by pressing the space bar on a computer keyboard, the dots in the two apertures started moving.

Seventy-five percent of the trials were 'cued trials,' $25 \%$ were 'catch trials' (Fig. 2). For the 'cued trials,' three different conditions were presented with equal probabilities, namely valid, invalid, and neutral trials. Hence, across trials cues were completely unpredictive with respect to the upcoming target location. In the valid and invalid trials, 200$3000 \mathrm{~ms}$ after trial start, the dots in one of the apertures changed their color to red. The color change lasted for $50 \mathrm{~ms}$ and served as the exogenous cue. A variable time interval $(0-1000 \mathrm{~ms})$ after cue-offset (cue-target ISI), the dots in either the same (valid condition) or the opposite (invalid condition) aperture underwent a direction change of $23 \mathrm{deg}$ and returned to their original direction after another $50 \mathrm{~ms}$. In the neutral trials, the color change occurred in both stimuli such that no particular location was cued. For all 'cued trials' the subjects' task was to detect the direction change and respond with a keypress ('H') as quickly as possible. Reaction times below $100 \mathrm{~ms}$ were considered anticipatory responses, reaction times above $1000 \mathrm{~ms}$ were counted as misses. The 'catch trials' were identical to the 'cued trials' except that no direction change (i.e., target) followed the cue, i.e., the dots continued to move in their original direction until trial end (signal absent trials). Thus, no response was required. 'Catch trials' were randomly interleaved with the 'cued trials' and served the purpose of preventing subjects from forming temporal expectancies and making anticipatory responses. After each trial, subjects received auditory feedback.

For each condition, RTs for correct 'cued trials' were sorted according to the cue-target ISI into 5 bins of $200 \mathrm{~ms}$ width (0-200, 200-400, 400-600, $600-800$, and $800-1000 \mathrm{~ms}$ ). For the statistical analysis of reaction times, a two-way ANOVA with the within-subject factors cueing (valid, invalid, neutral) and ISI (5 levels) was used. When appropriate, significance levels were corrected for violations of the sphericity assumption using the Greenhouse-Geyser method; however, original degrees of freedoms are reported.

\subsection{Experiment 2}

In Experiment 2, the same circular apertures and trial sequence as in Experiment 1 were used (Fig. 3). Here, each stimulus consisted of two superimposed populations of dots moving in opposite directions, resulting in a percept of transparent motion. The two dot populations always moved in the same opposite directions in the two apertures (45/225 deg or $135 / 315$ deg deviation from vertical). Stimulus parameters were identical to those used in Experiment 1 except for dot density. In Experiment 2, each individual surface contained only half the dots $\left(4\right.$ pixels $\left./ \mathrm{deg}^{2}\right)$ in order to keep the overall dot-density constant across experiments. To compensate for the reduced dot density in each single surface the duration of the cue color change was doubled to $100 \mathrm{~ms}$.

Using two transparent motion stimuli allowed the presentation of two different features (i.e., motion directions) superimposed at a single spatial location, and to repeat the same feature (i.e., same motion direction) at two different spatial locations. Since the color-cue and the directiontarget always occurred in only a single motion direction in a single dot pattern, we were able to independently manipulate the cue-validity with respect to location and feature (Fig. 4). Again, the cue was entirely unpredictive regarding the location and the direction of motion of the subsequent target.

For example, in the 'valid location, valid direction' condition the target occurred in the same location and in the same motion direction as the preceding cue. Analogously, the 'invalid location, valid direction' condition consisted of a target direction change occurring in the dot pattern opposite from the cued dot pattern, but in the population of dots moving in the same motion direction as the population of dots that served as the cue. The combinations of cue validity with respect to location (valid/invalid location) and motion direction (valid/invalid feature) yielded four different trial types. Additionally, a 'neutral condition' was presented in which the cue appeared simultaneously in one surface of each stimulus, such that no particular location or direction was cued. As in Experiment 1, the ratio of 'cued trials' and 'catch trials' was 3:1, cued surface, location and the directions of motion were randomized across trials.

Twelve naive subjects (age: 20-31, 5 female, 7 male) participated in two $1 \mathrm{~h}$ sessions conducted at different days. One subject was excluded from the data analysis because performance was below $50 \%$ in the catch trial condition ( $44 \%$ correct). As in the first experiment, trials were sorted into bins of $200 \mathrm{~ms}$ width according to the cue-target ISI. For the statistical
A

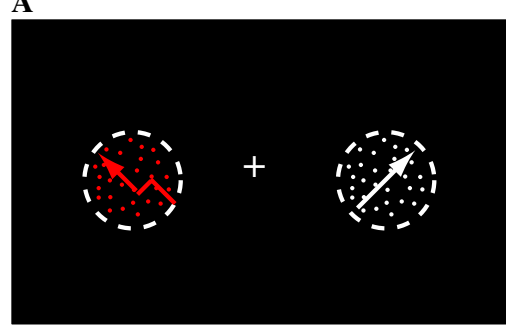

C

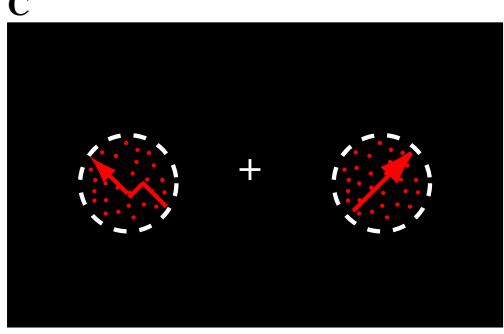

B

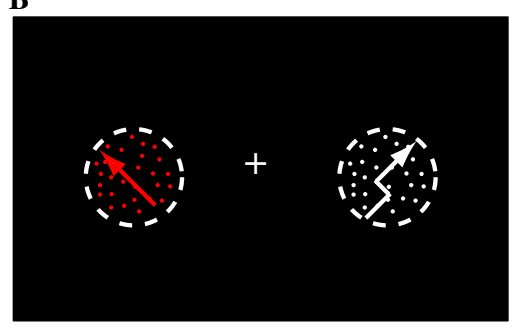

D

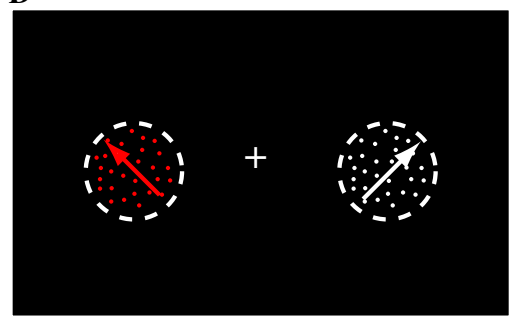

Fig. 2. Conditions for Experiment 1. For purpose of illustration the cue is drawn in the same frame as the target, which was not the case in the actual experiment (see Fig. 1). (A) Valid condition: cue and target in the same aperture. (B) Invalid condition: cue and target in different apertures. (C) Neutral condition: the cue appears in both apertures. (D) Catch trial: no target. The ratio of catch trials was $25 \%$, conditions (A-C) were presented with equal probabilities. The location of the cue and the two directions of motion were randomly varied across trials. 


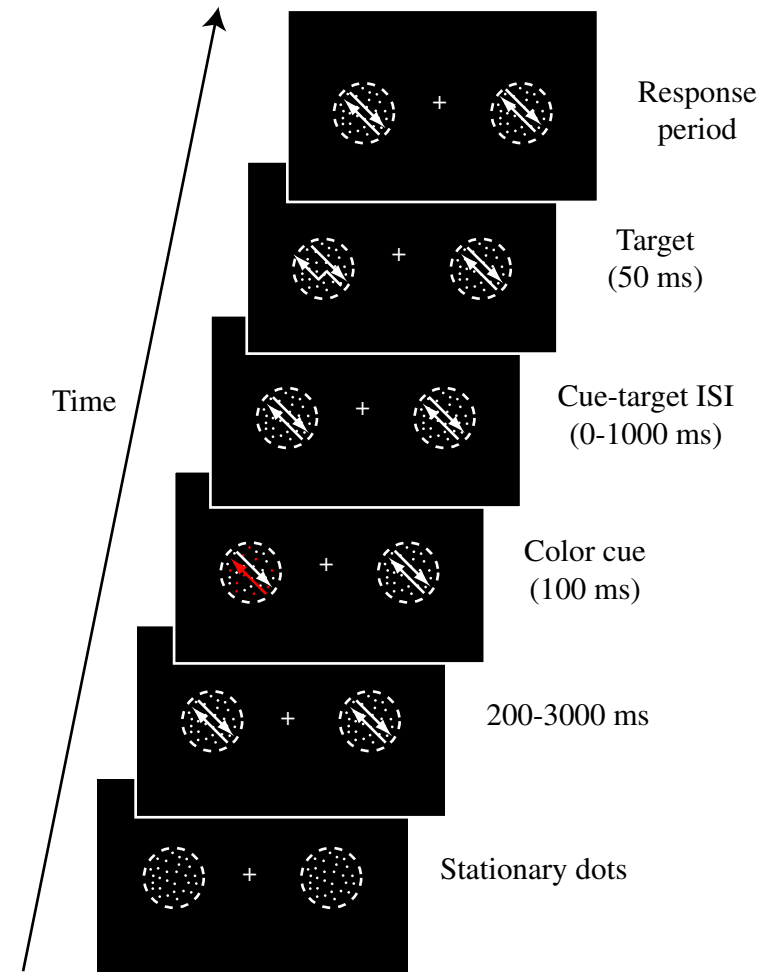

Fig. 3. Schematic trial sequence for Experiment 2. Two superimposed surfaces moving in opposite directions were presented to the left and right of fixation. After $200-3000 \mathrm{~ms}$ a color change in one surface served as the exogenous cue. Since the cue appeared only in a single direction of motion at a particular location we can independently manipulate the validity of the cue with respect to location and feature. The subjects' task was to respond as quickly as possible to the change in direction that could follow within $1000 \mathrm{~ms}$ after cue offset. In neutral trials, the cue was presented in one direction of each aperture, such that no particular location or feature was cued. In 'catch trials' no target followed the cue. The example illustrates a trial in the condition 'valid location, valid direction' since the target occurs in the cued aperture and in the cued direction of motion.

analysis of RT data, a three-way ANOVA with the within-subject factors location (valid, invalid), direction (valid, invalid), and ISI (5 levels) was used. When appropriate, significance levels were corrected using the Greenhouse-Geyser correction; however, original degrees of freedom are reported. RTs in the neutral condition were compared against RTs in the other cued trials in post-hoc comparisons.

\section{Results}

\subsection{Experiment 1}

In the first experiment, we investigated whether an exogenous color-cue affects processing of subsequent motion targets. Across subjects, performance for cued trials was $94.8 \%$, for catch trials $96.5 \%$.

Mean reaction times for Experiment 1 are shown in Fig. 5. The ANOVA for RTs revealed a significant main effect of ISI $(F(4,32)=8.35, p<0.01)$, indicating that RTs vary as a function of cue-target ISI. More importantly, the effects of exogenous cueing depend on the level of cue-target ISI, as reflected in the significant interaction between these factors $(F(8,64)=2.56, p<0.05)$. In trials with short cue-target ISIs (0-200 ms), subjects were faster in both the valid and neutral condition than in the invalid condition (462/470 ms vs. $496 \mathrm{~ms}$, respectively; $p<0.05$, NewmanKeuls). In contrast, for longer cue-target ISIs (200-400 and $400-600 \mathrm{~ms}$ ), this pattern first disappears and then reverses. This crossover effect is significant for ISIs between 400 and $600 \mathrm{~ms}$ with responses to targets after valid and neutral cues being on average $13 \mathrm{~ms}$ slower than responses to targets at uncued locations (436/433 ms vs. $422 \mathrm{~ms}$, respectively; $p<0.05$, Newman-Keuls). For longer cue-target ISIs, there was no significant difference between the conditions.

\subsection{Experiment 2}

In Experiment 2, we investigated location- and featurebased effects of exogenous cueing, using transparent motion stimuli. In this experiment, performance for cued trials $(76.9 \%)$ and catch trials $(82.1 \%)$ were lower compared to Experiment $1(t(18)=4.9, p<0.001$ for cued trials, and $t(18)=2.4, p<0.05$ for catch trials), and mean RTs across all conditions were slower $(444 \mathrm{~ms}$ vs. $507 \mathrm{~ms} ; t(18)=2.2$, $p<0.05)$ in Experiment 2 than Experiment 1. These differences probably reflect an increased target detection difficulty due to the presence of a second, overlapping dot surface.

Mean RTs for Experiment 2 are shown in Fig. 6. Along with the increased RTs compared to Experiment 1 goes an increase of the crossover latency in which facilitation changes to inhibition (600-800 ms bin). This agrees with the proposal that the onset of IOR might vary with the difficulty of the task (Lupiáñez, Milan, Tornay, Madrid, \& Tudela, 1997; Lupiáñez, Milliken, Solano, Weaver, \& Tipper, 2001). The reasoning is as follows: the more difficult the task, the more attention will be devoted to the target processing, and hence to the processing of the cue (Folk, Remington, \& Johnston, 1992); the more intensely attention will be allocated to the cue, the longer attention will dwell on it (Klein, 2000), and the longer facilitation will last after the exogenous cue.

An overall three-way ANOVA (location $\times$ direction $\times$ ISI) on RTs revealed a main effect of ISI $(F(4,40)=5.96$, $p<0.05)$, indicating that RTs vary as a function of cue-target ISI. A main effect of location $(F(1,10)=5.25, p<0.05)$ indicates that, on average, responses to targets at the cued location were faster than responses to targets at the uncued location ( $491 \mathrm{~ms}$ vs. $507 \mathrm{~ms}$ ). This effect is, on average, larger for targets with the cued feature as revealed by the interaction between location and direction $(F(1,10)=5.69$, $p<0.05)$. In addition, we obtain a significant interaction between location and ISI $(F(4,40)=7.32, p<0.001)$, showing that the effect of spatial cueing changes, on average, with ISI. Most importantly, the three-way interaction is also significant (location $\times$ direction $\times$ ISI, $F(4,40)=3.03$, $p=0.05)$. Post hoc comparisons revealed significant differences between the cueing conditions for the ISI bins 0-200, 200-400, and 600-800 ms. For the first and second ISI bin, 
A

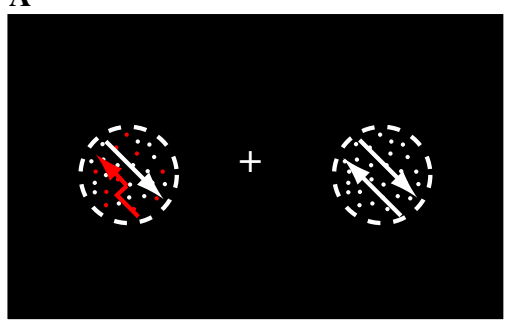

C

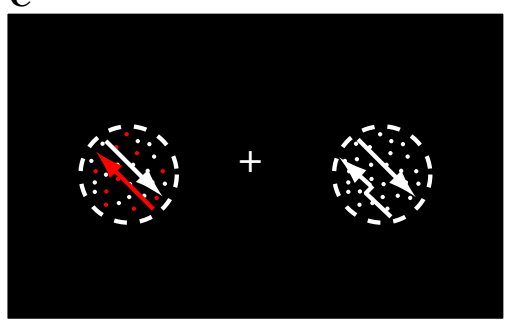

E

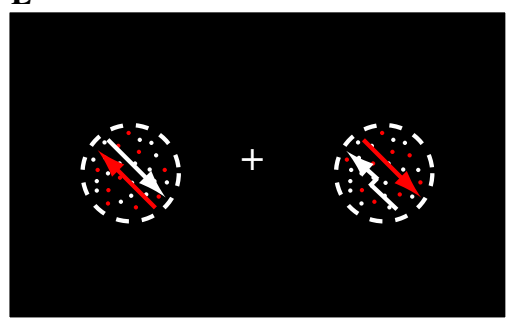

B

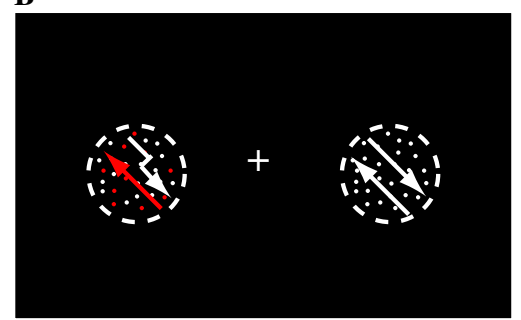

D

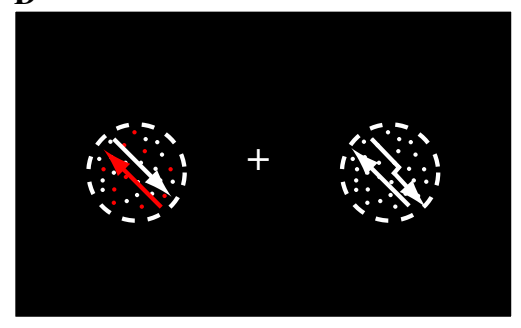

$\mathbf{F}$

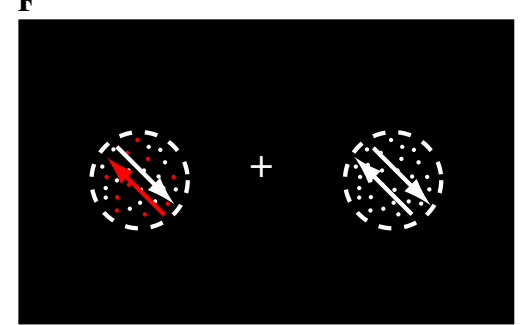

Fig. 4. Conditions in Experiment 2. (A) Valid location, valid direction. (B) Valid location, invalid direction. (C) Invalid location, valid direction. (D) Invalid location, invalid direction. (E) Neutral condition. (F) Catch trial. The ratio of catch trials was $25 \%$. Conditions (A-E) were presented with equal probability. Note that, in the actual experiment, the cue and the target were not presented at the same time.

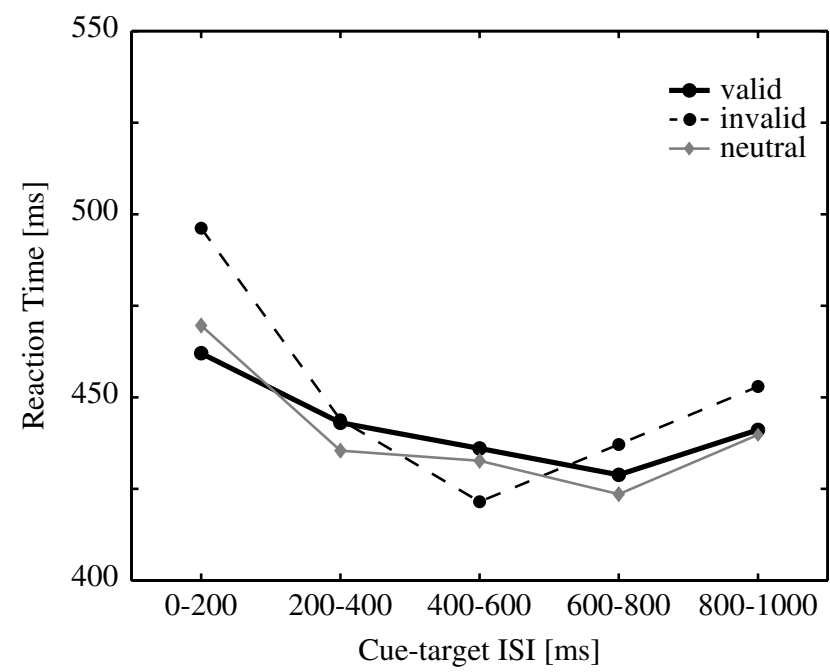

Fig. 5. Mean reaction times for Experiment 1. In conditions with short cue-target ISIs RTs are faster after both valid and neutral cues compared to invalid cues (466 ms vs. $496 \mathrm{~ms}$; 0-200 ms ISI). However, for longer cuetarget ISIs, this pattern reverses, and targets in the uncued dot pattern are responded to faster compared to both cued and neutral targets $(435 \mathrm{~ms}$ vs. $422 \mathrm{~ms}$; 400-600 ms ISI).

a separate two-way ANOVA with factors location and direction revealed a main effect of location $(F(1,10)$ $=10.77, p<0.01(0-200 \mathrm{~ms}$ ISI $) ; F(1,10)=6.42, p<0.05$
(200-400 ms ISI)), indicating that, on average, responses to targets in the cued location were faster than responses to targets in the uncued location. Moreover, we obtain a significant interaction between location $\times$ feature $(F(1,10)=8.82, p<0.05(0-200 \mathrm{~ms}$ ISI), $F(1,10)=11.28$, $p<0.01$ (200-400 ms ISI)). Post hoc comparisons show that RTs are fastest to targets appearing at the cued location and in the cued feature ('valid location, valid direction'), while there is no significant difference between the other cueing conditions $(496 \mathrm{~ms}$ vs. $565 \mathrm{~ms}$ for $0-200 \mathrm{~ms}$, $483 \mathrm{~ms}$ vs. $522 \mathrm{~ms}$ for $200-400 \mathrm{~ms} ; p<0.05$, NewmanKeuls). Additionally, there is a significant benefit for targets at the cued location and with the cued feature compared to the neutral condition for the ISI bin $0-200 \mathrm{~ms}$ ( $496 \mathrm{~ms}$ vs. $538 \mathrm{~ms} ; p<0.05$, Newman-Keuls). This difference vanishes in the subsequent ISI bin. A cross-over of RTs is evident in the $600-800 \mathrm{~ms}$ bin. Here, RTs to targets at the cued location are slower than RTs to targets at the uncued location $(483 \mathrm{~ms}$ vs. $456 \mathrm{~ms}, \quad F(1,10)=5.52$, $p<0.05$, main effect of location). Importantly, this effect does not depend on the cued feature $(F(1,10)=0.16$, $p=0.6$, interaction between feature and location). There is also no significant difference between RTs to targets at the cued location and RTs in the neutral condition for both the third (400-600 ms) and fourth ISI (600-800 ms) periods. 


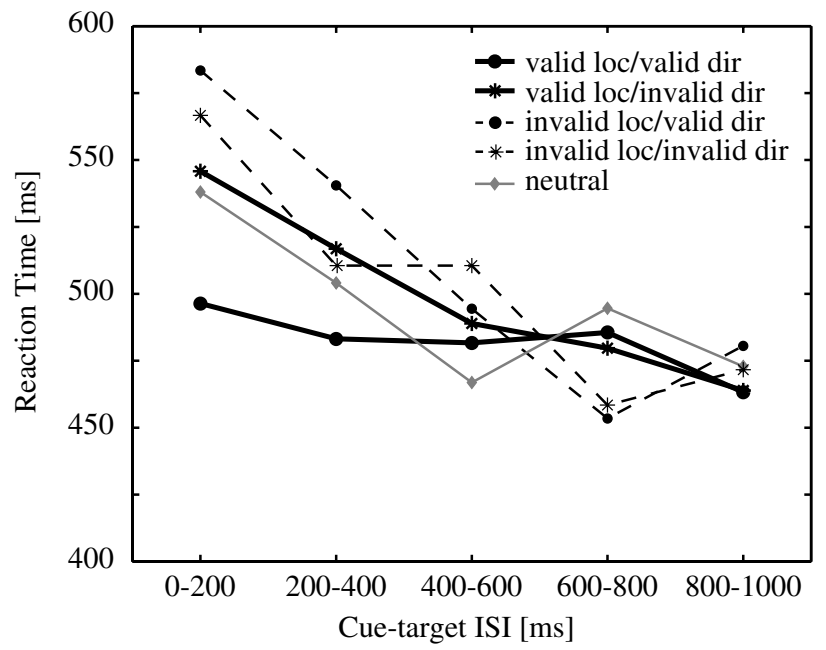

Fig. 6. Mean reaction times for experiment 2. At cue-target ISIs between 0-200 ms and 200-400 ms RTs are fastest if the target occurs in the previously cued location and direction ( $496 \mathrm{~ms}$ vs. $565 \mathrm{~ms}$ for $0-200 \mathrm{~ms} ; 483 \mathrm{~ms}$ vs. $522 \mathrm{~ms}$ for $200-400 \mathrm{~ms}$ ) compared to the other cueing conditions. While for the earliest targets, a benefit of the 'valid location, valid direction' compared to the neutral condition $(538 \mathrm{~ms})$ is present, this difference disappears between 200 and $400 \mathrm{~ms}$. At longer cue-target intervals (600$800 \mathrm{~ms}$ ), RTs are slower for targets appearing in the cued location compared to the uncued location ( $483 \mathrm{~ms}$ vs. $456 \mathrm{~ms}$ ). There is no difference between RTs in the neutral condition and RTs to validly cued targets in neither the third or fourth ISI period.

\section{Discussion}

In two experiments, we show spatial and feature-based effects of exogenous cueing on the processing of visual motion using an experimental design that does not confound cueing of location and cueing of feature. We find that, with short cue-target ISIs, detection of a change in direction of motion at the cued location is facilitated as evidenced by faster RTs to validly cued targets (Experiment 1). When further dissociating spatial and feature-based effects of the cue (Experiment 2), it becomes evident that this facilitation is feature-specific, such that targets occurring in the cued aperture (location) and in the cued direction of motion (feature) yield the fastest responses. In contrast, when the cue-target ISI is in the range of 400 $600 \mathrm{~ms}$ (Experiment 1) or 600-800 ms (Experiment 2), RTs to targets at the cued location are prolonged. This late inhibitory effect on RTs seems to be purely spatial.

By using a cue-target combination consisting of two different stimulus attributes processed in different visual pathways, namely a color change serving as a cue and a direction change constituting the target, we can infer that simple sensory interactions are not likely to cause the sequence of facilitatory and inhibitory effects of the cue. Furthermore, since we are not repeating the same feature for the cue and the target, we can exclude repetition blindness as an explanation for the effects in our experiments (Fox \& de Fockert, 2001). Rather, we interpret the findings as attention being initially reflexively drawn to the cued feature at the cued location, and later being oriented away from such tagged locations.

\subsection{Mechanisms and neuronal substrates}

Although the effects of exogenous cueing have been studied extensively using behavioral techniques, the underlying physiological processes remain unclear. So far, few studies have investigated the brain areas and mechanisms involved in automatic orienting as well as the physiological consequences of exogenous cueing on sensory processing.

\subsubsection{Initial attraction of attention}

In line with numerous previous studies, we find an initial facilitation of RTs to cued compared to uncued targets for short cue-target ISIs in both experiments. In Experiment 1, this initial difference does not seem to be due to a speed-up of RTs relative to the neutral condition (although the data show a trend in this direction), but seems caused primarily by prolonged responses to targets at the uncued location. In contrast, in the second experiment, RTs in the "valid location, valid feature' condition are considerably faster than the neutral and all other cueing conditions. This difference between the two experiments is surprising, and we can only speculate about the causes: Maybe processing of the neutral cue led to fast responses in the first experiment since task difficulty was low and attention was attracted in a split-focus fashion (e.g., Awh \& Pashler, 2000; McMains \& Somers, 2004) to both apertures with little or no cost after the neutral cue. Further experiments testing different neutral cues are needed to examine the facilitatory component after exogenous cueing relative to a neutral condition.

A number of recent studies employed transparent motion stimuli to investigate the effects of exogenous cueing on motion processing. They found that, after a salient change in one of two spatially superimposed, rotating surfaces performance in a subsequent discrimination task was strongly reduced for the uncued surface (e.g., Mitchell, Stoner, Fallah, \& Reynolds, 2003; Reynolds, Alborzian, \& Stoner, 2003; Valdés-Sosa, Cobo, \& Pinilla, 2000). This benefit in performance for discrimination in the cued surface lasted for a couple of hundreds of milliseconds. Thus, the facilitatory effect on performance has a similar time-course than the RT effect we observed in Experiment 2. The fact that the three studies cited above did not vary spatial location but presented the cue and target at fixation might have disguised a potential subsequent locationbased inhibition that would have affected both features similarly.

As has been proposed in computational models of bottom-up attentional processes (Itti \& Koch, 2000; Itti \& Koch, 2001), location-based facilitation of RTs with short cue-target ISIs could be interpreted as arising from a peak in activity in a feature-unspecific, retinotopically organized global 'salience map,' reflexively drawing attention to the most salient (i.e., cued) location. This 'salience map' is thought to receive input from multiple feature-specific 
maps that each encode spatial contrast in one specific feature dimension, like orientation, color, or motion. Directing attention to the location corresponding to the peak activity in such a feature-unspecific salience map would yield a purely spatial-based attentional advantage. Evidence for representation of salience, not stimulus features per se, has been found for neurons in the pulvinar (Robinson \& Petersen, 1992), parietal cortex (Gottlieb, Kusunoki, \& Goldberg, 1998), in the frontal eye fields (FEF) (Thompson \& Schall, 2000; Thompson, 2001), and in the superior colliculus (SC) (Kustov \& Robinson, 1996). However, to account for a feature-based component in the initial orienting of attention as found in Experiment 2 one would need to assume an additional, feature-specific mechanism, e.g., incorporation of feature information in the global saliency map or interactions with distributed feature-specific computations of salience (e.g., Desimone \& Duncan, 1995; Hamker, 2004).

\subsubsection{Inhibition of return}

IOR has been proposed to be a crucial mechanism of attentional orienting in that it prevents attention from permanently focusing on the most salient stimulus (Itti \& Koch, 2001; Klein, 1988). According to this notion, attention is able to shift to different stimuli with decreasing saliency in the visual scene by transient inhibition of neurons in the 'salience map' encoding the attended stimulus (Itti \& Koch, 2000, 2001).

Experiments 1 and 2 both demonstrated IOR for longer cue-target ISIs when targets appeared at the cued location. Consistently across the experiments, this disadvantage for the previously cued location seems to be primarily caused by a benefit for the uncued location, since RTs to targets in the uncued location are considerably faster than RTs to targets after both spatially valid and neutral cues. Thus, instead of being a true inhibition of the attended stimulus, the effect might rather be interpreted as a facilitation of previously unattended locations. On the other hand, our neutral cue, consisting of a salient change in both apertures, might have led to similar inhibitory processes than the valid cue. To disentangle these opposing interpretations further experiments using a different neutral cue, e.g., a change at fixation, need to be conducted.

The finding that IOR seems to operate in a purely spatial manner has immediate functional plausibility. ${ }^{2}$ When searching for an item with defining characteristics (e.g., a certain color) in a cluttered visual scene spatial IOR biases the system toward processing of new locations if the item has not been found at the previously attended location. This mechanism seems crucial for efficient attentional employment. In contrast, it would seem an inappropriate strategy if the attended feature, which defines the target item, would be inhibited across the entire visual field by a global feature-based IOR.

\footnotetext{
${ }^{2}$ We thank one anonymous reviewer for pointing out this issue.
}

Single-unit recordings in the lateral intraparietal (LIP) area in awake behaving monkeys have revealed a potential neural correlate of a transient spatial-based inhibition (Bisley \& Goldberg, 2003). In this study, typical effects of exogenous cueing on behavior (lower contrast thresholds with short ISIs, higher contrast thresholds with long ISIs for targets at the cued location) correlate with the ensemble activity in LIP. Shortly after a transient cue-related activity, the LIP population response is increased for neurons with receptive fields (RF) responding to the cued region compared to neurons whose RFs are located at the opposite target location. After a period of $80-90 \mathrm{~ms}$ without any significant difference between the two population responses, the firing rate of the neurons coding the cued region decreases below the firing rate of those coding the opposite target location.

Along the lines of the motor theory of IOR (Rafal, Calabresi, Brennan, \& Sciolto, 1989; Sapir, Soroker, \& Berger, 1999), single unit studies in the superior colliculus (SC), a critical node in the visual orienting pathway, propose a collicular contribution to IOR (Dorris, Klein, Everling, \& Munoz, 2002; Fecteau, Bell, \& Munoz, 2004). For short cue-target intervals, Fecteau et al. (2004) showed that the initial facilitation of saccadic RTs is accompanied by an enhanced neural response to the target in the SC. Similarly, prolonged RTs correlate with a suppression of the target-related responses in the SC (Dorris et al., 2002; Fecteau et al., 2004) and reduced target-related responses at the cued location have been found in the event-related scalp potential (ERP) recorded in humans. At long cue-target intervals, P1 amplitudes are reduced when targets appear at the cued location, suggesting that exogenous cueing can influence sensory processing in the extrastriate cortex (Hopfinger \& Mangun, 1998; McDonald, Ward, \& Kiehl, 1999). Using event-related fMRI IOR has been linked to areas commonly activated during oculomotor/motor planning (frontal and supplementary eye fields) (Lepsien \& Pollmann, 2002; Mayer, Seidenberg, Dorflinger, \& Rao, 2004) as well as attentional orienting (posterior parietal, superior and middle temporal, anterior cingulate and thalamic areas) (Mayer et al., 2004).

\subsection{Perceptual effects vs. shifts of criterion}

Traditionally, the effects of stimulus-driven or bottomup capture of attention have been interpreted as reflecting perceptual effects. Recently, an alternative explanation has been advanced, namely a simple change in criterion for targets at attended vs. unattended locations (Eckstein, Thomas, Palmer, \& Shimozaki, 2000; Verghese, 2001). In case of the Posner cueing paradigm, Eckstein, Shimozaki, and Abbey (2002) propose an ideal-observer model that weights the information at cued and uncued locations as a function of the cue validity. They find close agreement of psychophysical data with the model and no evidence for a change in the quality of the visual signal, which suggests a 
change in criterion rather than a perceptual benefit of attention. While RT data cannot distinguish between shifts of sensitivity vs. criterion, one should note that simple weighting of information according to the cue-validity cannot account for effects after uninformative precues as used in this study. Using a signal-detection measure of perceptual sensitivity in a design with unpredictive cues, Handy et al. (1999) showed that the typical pattern of RTs, namely early facilitation and later IOR, is also found for sensitivity measures like $d^{\prime}$ and $A^{\prime}$. Similarly, an irrelevant singleton in a search array seems to attract attention such that target detectability seems reduced (Theeuwes, Kramer, \& Kingstone, 2004). Finally, Carrasco, Ling, and Read (2004) recently demonstrated that uninformative exogenous cues alter the perceived contrast of the cued items, at least with a short cue-target ISI. Unfortunately, our design does not allow us to determine the signal detection measures $d^{\prime}$ and $\beta$ in order to directly test for changes in sensitivity vs. decision criterion. While it is straightforward to calculate hit rates for the various cue-target ISIs for the different signal present conditions, it is impossible (except for the neutral cueing condition) to categorize false alarms with respect to the various valid or invalid cueing conditions. Moreover, there is no obvious way to sort the false alarms into the cue-target ISI bins. Clearly, further experiments will be needed to dissociate changes in sensitivity from changes in criterion over time in an exogenous cueing task.

\section{Summary}

In summary, we show spatial and feature-based influences of exogenous cueing on motion processing. By using a cue-target combination that avoids simple sensory interactions we infer that exogenous automatic attentional processes can best account for the results. With short cuetarget ISIs, we find a benefit for targets at the valid location and with the valid feature, probably reflecting attentional orienting to the cued item. In contrast, with long cue-target ISIs, responses to targets are slower when they occur at the cued location irrespective of the cued feature. This finding underlines the notion that IOR might facilitate orienting to novel spatial locations.

\section{Acknowledgments}

We thank Annette Heinrich for testing some of the subjects in Experiment 2 and for providing help with the figures. This study was funded in part by support from the state of Lower Saxony.

\section{References}

Awh, E., \& Pashler, H. (2000). Evidence for split attentional foci. Journal of Experimental Psychology: Human Perception and Performance, 26(2), 834-846.

Bisley, J., \& Goldberg, M. (2003). Neuronal activity in the lateral intraparietal area and spatial attention. Science, 299(5603), 81-86.
Blaser, E., Pylyshyn, Z., \& Holcombe, A. (2000). Tracking an object through feature space. Nature, 408, 196-199.

Carrasco, M., Ling, S., \& Read, S. (2004). Attention alters appearance. Nature Neuroscience, 7(3), 308-313.

Christ, S., McCrae, C., \& Abrams, R. (2002). Inhibition of return in static and dynamic displays. Psychonomic Bulletin \& Review, 9(1), 80-85.

Clifford, C. (2002). Perceptual adaptation: Motion parallels orientation. Trends in Cognitive Sciences, 6(3), 136-143.

Desimone, R., \& Duncan, J. (1995). Neural mechanisms of selective visual attention. Annual Review of Neuroscience, 18, 193-222.

Dorris, M., Klein, R., Everling, S., \& Munoz, D. (2002). Contribution of the primate superior colliculus to inhibition of return. Journal of Cognitive Neuroscience, 14(8), 1256-1263.

Eckstein, M., Shimozaki, S., \& Abbey, C. (2002). The footprints of visual attention in the posner cueing paradigm revealed by classification images. Journal of Vision, 2(1), 25-45.

Eckstein, M. P., Thomas, J. P., Palmer, J., \& Shimozaki, S. S. (2000). A signal detection model predicts the effects of set size on visual search accuracy for feature, conjunction, triple conjunction, and disjunction displays. Perception \& Psychophysics, 62(3), 425-451.

Fecteau, J., Bell, A., \& Munoz, D. (2004). Neural correlates of the automatic and goal-driven biases in orienting spatial attention. Journal of Neurophysiology, 92(3), 1728-1737.

Felleman, D., \& Van Essen, D. (1991). Distributed hierarchical processing in the primate cerebral cortex. Cerebral Cortex, 1, 1-47.

Folk, C., Remington, R., \& Johnston, J. (1992). Involuntary covert orienting is contingent on attentional control settings. Journal of Experimental Psychology: Human Perception and Performance, 18(4), 1030-1044.

Fox, E., \& de Fockert, J. (2001). Inhibitory effects of repeating color and shape: Inhibition of return or repetition blindness? Journal of Experimental Psychology: Human Perception and Performance, 27(4), 798812.

Gottlieb, J., Kusunoki, M., \& Goldberg, M. (1998). The representation of visual salience in monkey parietal cortex. Nature, 391(6666), 481-484.

Hamker, F. H. (2004). A dynamic model of how feature cues guide spatial attention. Vision Research, 44, 501-521.

Handy, T., Jha, A., \& Mangun, G. (1999). Promoting novelty in vision: Inhibition of return modulates perceptual-level processing. Psychological Science, 10(2), 157-161.

Hopfinger, J., \& Mangun, G. (1998). Reflexive attention modulates processing of visual stimuli in human extrastriate cortex. Psychological Science, 9(6), 441-447.

Itti, L., \& Koch, C. (2000). A saliency-based search mechanism for overt and covert shifts of visual attention. Vision Research, 40(10-12), 1489-1506.

Itti, L., \& Koch, C. (2001). Computational modelling of visual attention. Nature Review Neuroscience, 2(3), 194-203.

Kanwisher, N. (1987). Repetition blindness: Type recognition without token individuation. Cognition, 27(2), 117-143.

Klein, R. (1988). Inhibitory tagging system facilitates visual search. Nature, 334(6181), 430-431.

Klein, R. (2000). Inhibition of return. Trends in Cognitive Sciences, 4, 138147.

Kustov, A., \& Robinson, D. (1996). Shared neural control of attentional shifts and eye movements. Nature, 384(6604), 74-77.

Kwak, H.-W., \& Egeth, H. (1992). Consequences of allocating attention to locations and to other attributes. Perception \& Psychophysics, 51, 455-464.

Law, M., Pratt, J., \& Abrams, R. (1995). Color-based inhibition of return. Perception \& Psychophysics, 57, 402-408.

Leek, E., Reppa, L., \& Tipper, S. (2003). Inhibition of return for objects and locations in static displays. Perception \& Psychophysics, 65(3), 388-395.

Lepsien, J., \& Pollmann, S. (2002). Covert reorienting and inhibition of return: An event-related fMRI study. Journal of Cognitive Neuroscience, 14(2), 127-144.

Lupiáñez, J., Milan, E., Tornay, F., Madrid, E., \& Tudela, P. (1997). Does IOR occur in discrimination tasks. Yes, it does, but later. Perception \& Psychophysics, 59(8), 1241-1254. 
Lupiáñez, J., Milliken, B., Solano, C., Weaver, B., \& Tipper, S. (2001). On the strategic modulation of the time course of facilitation and inhibition of return. Quarterly Journal of Experimental Psychology, 54(3), 753-773.

Mayer, A., Seidenberg, M., Dorflinger, J., \& Rao, S. (2004). An eventrelated fMRI study of exogenous orienting: Supporting evidence for the cortical basis of inhibition of return? Journal of Cognitive Neuroscience, 16(7), 1262-1271.

McAuliffe, J., Pratt, J., \& O’Donnell, C. (2001). Examining location-based and object-based components of inhibition of return in static displays. Perception \& Psychophysics, 63(6), 1072-1082.

McDonald, J., Ward, L., \& Kiehl, K. (1999). An event-related brain potential study of inhibition of return. Perception \& Psychophysics, 61(7), 1411-1423.

McMains, S., \& Somers, D. (2004). Multiple spotlights of attentional selection in human visual cortex. Neuron, 42(4), 677-686.

Mitchell, J., Stoner, G., Fallah, M., \& Reynolds, J. (2003). Attentional selection of superimposed surfaces cannot be explained by modulation of the gain of color channels. Vision Research, 43(12), 1323-1328.

Muller, J., Metha, A., Krauskopf, J., \& Lennie, P. (1999). Rapid adaptation in visual cortex to the structure of images. Science, 285(5432), 1405-1408.

Posner, M. (1980). Orienting of attention. Quarterly Journal of Experimental Psychology, 32, 3-25.

Posner, M., \& Cohen, Y. (1984). Components of visual orienting. In H. Bouma \& D. Bouwhuis (Eds.), Attention and performance $X$ (pp. 531555). Hillsdale, NJ: Erlbaum.

Rafal, R., Calabresi, P., Brennan, C., \& Sciolto, T. (1989). Saccade preparation inhibits reorienting to recently attended locations. Journal of Experimental Psychology: Human Perception and Performance, 15(4), 673-685.

Reynolds, J., Alborzian, S., \& Stoner, G. (2003). Exogenously cued attention triggers competitive selection of surfaces. Vision Research, 43(1), 59-66.

Riggio, L., Patteri, I., \& Umilta, C. (2004). Location and shape in inhibition of return. Psychological Research, 68, 41-54.
Robinson, D., \& Petersen, S. (1992). The pulvinar and visual salience. Trends in Neurosciences, 15(4), 127-132.

Sàenz, M., Burac̊as, G., \& Boynton, G. (2003). Global feature-based attention for motion and color. Vision Research, 43(6), 629-637.

Sapir, A., Soroker, N., \& Berger, A. (1999). Inhibition of return in spatial attention: Direct evidence for collicular generation. Nature Neuroscience, 2(12), 1053-1054.

Taylor, T., \& Klein, R. (1998). Inhibition of return to color: A replication and nonextension of Law, Pratt, \& Adams (1995). Perception \& Psychophysics, 60, 1452-1456.

Theeuwes, J., Kramer, A., \& Kingstone, A. (2004). Attentional capture modulates perceptual sensitivity. Psychonomic Bulletin \& Review, 11(3), $551-554$.

Thompson, K., \& Schall, J. (2000). Antecedents and correlates of visual detection and awareness in macaque prefrontal cortex. Vision Research, 40(10-12), 1523-1538.

Thompson, K. G. (2001). From attention to action in frontal cortex. In J. Braun, C. Koch, \& J. Davies (Eds.), Visual attention and cortical circuits (pp. 137-157). MA: MIT Press.

Tipper, S., Driver, J., \& Weaver, B. (1991). Object-centred inhibition of return of visual attention. Quarterly Journal of Experimental Psychology. A, Human Experimental Psychology, 43(2), 289-298.

Tipper, S., Weaver, B., Jerreat, L., \& Burak, A. (1994). Object-based and environment-based inhibition of return of visual attention. Journal of Experimental Psychology: Human Perception and Performance, 20(3), 478-499.

Ungerleider, L., \& Mishkin, M. (1982). Two cortical visual systems. In D. Ingle, M. Goodale, \& R. Mansfield (Eds.), Analysis of visual behavior (pp. 49-86). MA: MIT Press.

Valdés-Sosa, M., Cobo, A., \& Pinilla, T. (2000). Attention to object files defined by transparent motion. Journal of Experimental Psychology: Human Perception and Performance, 26(2), 488-505.

Verghese, P. (2001). Visual search and attention: a signal detection theory approach. Neuron, 31(4), 523-535. 


\subsection{The time course of shifting visual attention}

The following contribution is a short review of a recent article by Khayat et al. (2006) entitled "Attention lights up new object representations before the old ones fade away" which has been published in the Journal of Neuroscience. The reviewed paper investigates attentional modulation during shifts of visual attention, using multi-unit recordings in the primary visual cortex.

The time course of attentional modulation during shifts of attention represents an important issue in current research. The reviewed study is among the first to provide a detailed analysis of the dynamics of attentional modulation. 
Editor's Note: These short reviews of a recent paper in the Journal, written exclusively by graduate students or postdoctoral fellows, are intended to mimic the journal clubs that exist in your own departments or institutions. For more information on the format and purpose of the Journal Club, please see http://www.jneurosci.org/misc/ifa_features.shtml.

\title{
The Time Course of Shifting Visual Attention
}

\author{
Laura Busse and Steffen Katzner \\ Cognitive Neuroscience Laboratory, German Primate Center, 37707 Göttingen, Germany \\ Review of Khayat et al. (http://www.jneurosci.org/cgi/content/full/26/1/138)
}

At any instant, we are confronted with more information than we can fully process, because our sensory and cognitive resources are limited. To cope with this avalanche we use selective attention to enhance information from the environment that we care about and suppress the rest. Extracellular single-unit recordings in the visual cortex of awake, behaving macaques have provided detailed information about the mechanisms of "sustained" attention. In these studies, attention was directed to a visual stimulus or a stream of visual stimuli for up to several seconds. Little is known, though, about "shifts" in attention. This is an important gap in our knowledge because attention must operate on millisecond time scales. Imagine driving along a busy road. While keeping your eyes on the road, you also have to attend to cars on each side, pedestrians crossing the street, upcoming signs and changing traffic lights. In such a situation, accident-free driving is only possible because we are able to quickly shift our focus of attention, thereby temporarily "lightening up" representations of the most relevant objects in the visual scene.

A recent study by Khayat et al. (2006) in The Journal of Neuroscience (http:// www.jneurosci.org/cgi/content/full/26/1/ 138) addresses how this might be achieved. The authors recorded multiunit

Received Feb. 1, 2006; accepted Feb. 17, 2006.

Correspondence should be addressed to Laura Busse, German Primate Center, Cognitive Neuroscience Laboratory, Kellnerweg 4, 37707 Göttingen, Germany. E-mail: Ibusse@gwdg.de.

D0I:10.1523/JNEUROSCI.0459-06.2006

Copyright $\odot$ 2006 Society for Neuroscience $\quad$ 0270-6474/06/263885-02\$15.00/0 activity from the primary visual cortex (V1) in two macaque monkeys while the animals performed a variant of the "curve-tracking" task, an elegant paradigm developed by Roelfsema et al. (1998) (Fig. 1A). On each trial, the monkey was presented with two curved lines, only one of which was connected via a small segment to the fixation point [Khayat et al., 2006, their Fig. 1a (http://www.jneurosci.org/cgi/content/full/26/1/138/FIG1)]. The monkey's task was to maintain its gaze on the fixation point for $800 \mathrm{~ms}$ and then saccade to the end of the target curve, the line connected to the fixation point. Roelfsema et al. (1998) have shown previously that neurons in $\mathrm{V} 1$ respond more strongly to such task-relevant curves, compared with distractor curves (i.e., curves that are task-irrelevant because they are not connected to the fixation point) [see also Khayat et al., 2006, their Fig. 2a-d (http://www.jneurosci.org/cgi/ content/full/26/1/138/FIG2)]. This selective modulation has been interpreted as a neuronal correlate of visual attention being directed to the target curve.

Khayat et al. (2006) extended the curve-tracking task to investigate the dynamics of attention. In addition to the "normal trials" described above, they introduced "switch trials," in which the monkey had to shift attention during the course of a trial (Fig. $1 B$ ), because the connection to the fixation point was changed while the monkey was waiting to saccade. Hence, the distractor curve became a target, and vice versa, thus changing the destination of the upcoming sac- cade [Khayat et al., 2006, their Fig. $1 b$ (http://www.jneurosci.org/cgi/content/ full/26/1/138/FIG1)]. The authors report that activity of neurons in area $\mathrm{V} 1$ reflects this change of behavioral relevance on a rapid time scale [Khayat et al., 2006, their Fig. $2 e-g$ (http://www.jneurosci.org/cgi/ content/full/26/1/138/FIG2)]. Remarkably, enhancement of responses caused by shifting attention to the new target curve occurred $\sim 60$ ms earlier than suppression of responses caused by removal of attention from the new distractor [Khayat et al., 2006, their Fig. 3 (http://www.jneurosci. org/cgi/content/full/26/1/138/FIG3)]. The $60 \mathrm{~ms}$ transition period, in which attention is already allocated to a new object before it is disengaged from a previously attended one, is incompatible with serial models of attentional shifts in which attention first has to be disengaged from an object before it can be shifted and allocated to another object (Shulman et al., 1979). Although Khayat et al. (2006) provide conclusive evidence against such serial models, further experiments are needed to test other models of attention that might account for the observed temporal pattern, such as split foci (McMains and Somers, 2004) or widening of the attentional focus (Müller et al., 2003).

To allow a direct comparison of neural responses during switch and normal trials the putative signal to switch attention had to occur at a fixed time $(400 \mathrm{~ms}$ in this case) after the trial begin, making the onset of the switch signal predictable. Hence, after $400 \mathrm{~ms}$, the animals were left without doubt about the task demands in the on- 
going trial ("switch" or "stay focused"). Primates have a precise internal representation of trial timing (Janssen and Shadlen, 2005), and the data of Khayat et al. (2006) suggest that their animals made use of it. In particular, responses during normal trials, in which no switch occurred, seem to decrease strongly after $\sim 550 \mathrm{~ms}$ [Khayat et al., 2006, their Fig. 3a, dotted lines (http://www.jneurosci.org/cgi/ content/full/26/1/138/FIG3)], suggesting that the monkeys' level of attention dropped after they had acquired some certainty that they were performing a normal trial. Because the authors compute the latency of attentional modulation using the difference of activity between switch and normal trials, the anticipation of the switch time might affect their estimates of absolute latencies. However, these absolute latencies do not alter their main finding, namely the relative temporal relationship between attentional enhancement and suppression.

It is remarkable that the size of the attentional enhancement after shifting attention to the new target curve seems to exceed the effect of short-term adaptation during normal trials [Khayat et al., 2006, their Fig. $3 a$, red solid line vs black dotted line (http://www.jneurosci.org/cgi/content/full/26/1/138/FIG3)]. However, because activity drops during normal trials, the extra attentional benefit for new targets is difficult to interpret. Here, it will be interesting to investigate, in further experiments, whether this additional enhancement also holds true in designs avoiding temporal predictability. This, in turn, would raise intriguing questions about the interaction between short-term adaptation and attention.

In summary, Khayat et al. (2006) provide the first detailed analyses of the temporal dynamics of attentional modulation during shifts of attention in visual area V1. Their data convincingly demonstrate that shifts of attention cannot simply be attributed to serial subprocesses that are executed sequentially but that attention can be allocated quickly to a new object even before it is disengaged from the previously attended one.

\section{References}

Janssen P, Shadlen M (2005) A representation of the hazard rate of elapsed time in macaque area LIP. Nat Neurosci 8:234-241.

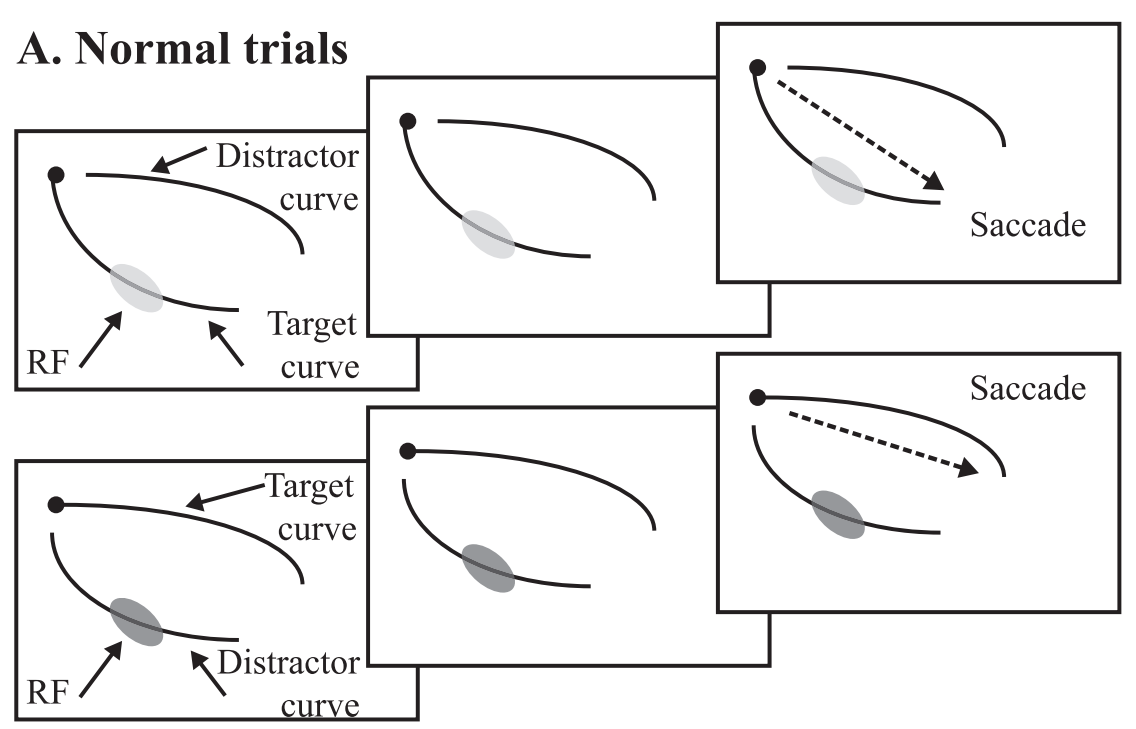

\section{B. Shift trials}

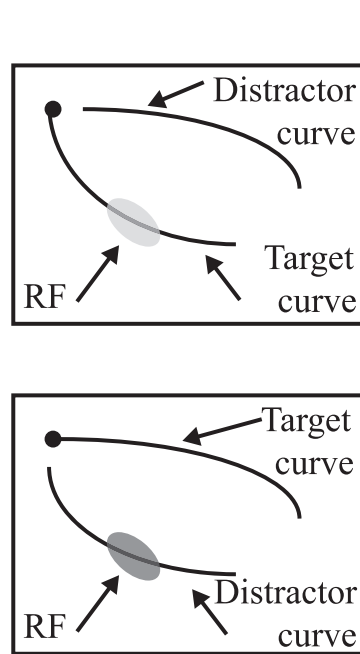

Stimulus onset Shift Cue $(\mathrm{p}=25 \%)$

$0 \mathrm{~ms}$

$400 \mathrm{~ms}$
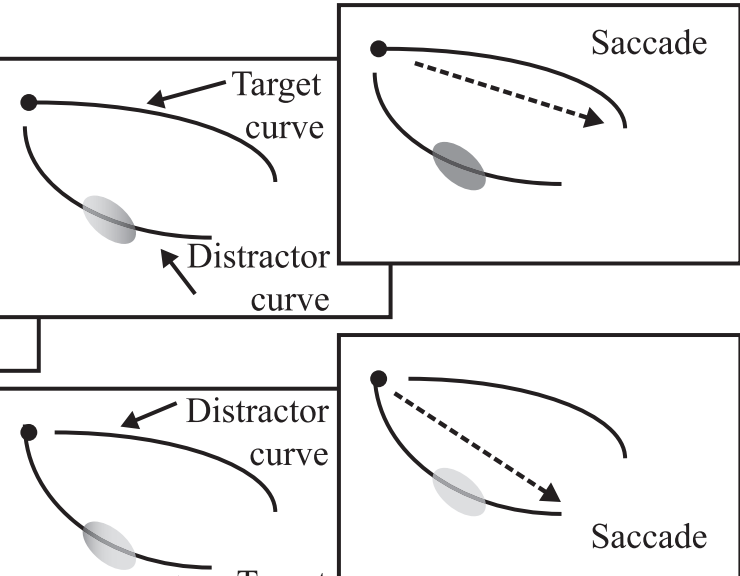

Saccade

Figure 1. Curve-tracing task in normal ( $\boldsymbol{A}$ ) and shift trials $(\boldsymbol{B})$. The shaded ellipse illustrates a V1 receptive field (RF). $\boldsymbol{A}$, During normal trials, the monkey fixates on a fixation point (black circle) for $800 \mathrm{~ms}$ before making a saccade to the end of the line connected to the fixation point (target curve). Roelfsema et al. (1998) have demonstrated an increase in V1 activity if the curve segment inside the RF belongs to the target versus distractor curve (indicated by light gray and dark gray shading, respectively). $\boldsymbol{B}$, During shift trials, the small line segment connecting the target curve to the fixation point is switched, thereby interchanging target and distractor curves. Khayat et al. (2006) show that this change of behavioral relevance is reflected in the activity of V1 neurons, leading to a fast and strong enhancement, only later followed by suppression (indicated by the gradients in the shading).

Khayat PS, Spekreijse H, Roelfsema PR (2006) Attention lights up new object representations before the old ones fade away. J Neurosci 26:138-142.

McMains SA, Somers DC (2004) Multiple spotlights of attentional selection in human visual cortex. Neuron 42:677-686.

Müller NG, Bartelt OA, Donner TH, Villringer A, Brandt SA (2003) A physiological correlate of the "Zoom Lens" of visual attention. J Neurosci 23:3561-3565.

Roelfsema PR, Lamme VA, Spekreijse H (1998) Object-based attention in the primary visual cortex of the macaque monkey. Nature 395:376-381.

Shulman GL, Remington RW, McLean JP (1979) Moving attention through visual space. J Exp Psychol Hum Percept Perform 5:522-526. 


\section{Chapter 3}

\section{Summary}

In summary, this work examines cognitive influences on the processing of visual motion information. Recording extra-cellular activity from individual, motion-sensitive neurons in macaque area MT, we have provided the first single-unit evidence for an object-based transfer of attention between different features of a visual stimulus. The behavioral experiments investigate consequences of dividing attention between visual features, mechanisms mediating behavioral benefits of signal anticipation, and the time course of automatic shifts of attention. Finally, a short review discusses recent electrophysiological investigations of the dynamics of visual attention. Together, these contributions show that cognitive factors strongly modulate the processing of visual motion. 


\section{Bibliography}

Albright, T. D. (1984). Direction and orientation selectivity of neurons in visual area MT of the macaque. Journal of Neurophysiology, 52(6):1106-1130.

Awh, E. and Pashler, H. (2000). Evidence for split attentional foci. Journal of Experimental Psychology: Human Perception and Performance, 26(2):834-846.

Beauchamp, M. S., Cox, R. W., and DeYoe, E. A. (1997). Graded effects of spatial and featural attention on human area MT and associated motion processing areas. Journal of Neurophysiology, 78:516-520.

Bichot, N. P., Rossi, A. F., and Desimone, R. (2005). Parallel and serial neural mechanisms for visual search in macaque area V4. Science, 308(5721):529-534.

Blake, R. and Sekuler, R. (2005). Perception. McGraw-Hill, New York, 5th edition.

Blaser, E., Pylyshyn, Z. W., and Holcombe, A. O. (2000). Tracking an object through feature space. Nature, 408(6809):196-199.

Born, R. T. and Bradley, D. C. (2005). Structure and function of visual area MT. Annual Review of Neuroscience, 28:157-189.

Brefczynski, J. A. and DeYoe, E. A. (1999). A physiological correlate of the 'spotlight' of visual attention. Nature Neuroscience, 2(4):370-374.

Britten, K. H. (2004). The middle temporal area: motion processing and the link to perception. In Chalupa, L. M. and Werner, J. F., editors, The Visual Neurosciences, pages 1203-1216. MIT Press, Cambridge, MA.

Britten, K. H., Shadlen, M. N., Newsome, W. T., and Movshon, J. A. (1992). The analysis of visual motion: a comparison of neuronal and psychophysical performance. Journal of Neuroscience, 12(12):4745-4765. 
Chalupa, L. M. and Werner, J. S., editors (2003). The Visual Neurosciences. MIT Press, Cambridge, MA.

Corbetta, M., Miezin, F. M., Dobmeyer, S., Shulman, G. L., and Petersen, S. E. (1990). Attentional modulation of neural processing of shape, color, and velocity in humans. Science, 248(4962):1556-1559.

Cutzu, F. and Tsotsos, J. K. (2003). The selective tuning model of attention: psychophysical evidence for a suppressive annulus around an attended item. Vision Research, 43(2):205-219.

DeAngelis, G. C. and Newsome, W. T. (1999). Organization of disparity-selective neurons in macaque area MT. Journal of Neuroscience, 19(4):1398-1415.

Duncan, J. (1984). Selective attention and the organization of visual information. Journal of Experimental Psychology: General, 113(4):501-517.

Egly, R., Driver, J., and Rafal, R. D. (1994). Shifting visual attention between objects and locations: evidence for normal and parietal lesion subjects. Journal of Experimental Psychology: General, 123(2):161-177.

Eriksen, C. W. and St. James, J. D. (1986). Visual attention within and around the field of focal attention: a zoom lens model. Perception \& Psychophysics, 40(4):225-240.

Felleman, D. J. and Van Essen, D. C. (1991). Distributed hierarchical processing in the primate cerebral cortex. Cerebral Cortex, 1(1):1-47.

Gattass, R. and Gross, C. G. (1981). Visual topography of striate projection zone (MT) in posterior superior temporal sulcus of the macaque. Journal of Neurophysiology, 46(3):621-638.

Ghose, G. M. and Maunsell, J. H. (2002). Attentional modulation in visual cortex depends on task timing. Nature, 419(6907):616-620.

Hendry, S. H. C. and Reid, R. C. (2000). The koniocellular pathway in primate vision. Annual Review of Neuroscience, 23:127-153.

Hopf, J. M., Boehler, C. N., Luck, S. J., Tsotsos, J. K., Heinze, H. J., and Schoenfeld, M. A. (2006a). Direct neurophysiological evidence for spatial suppression surrounding the focus of attention in vision. Proceedings of the National Academy of Sciences, 103(4):1053-1058.

Hopf, J. M., Boelmans, K., Schoenfeld, M. A., Luck, S. J., and Heinze, H. J. (2004). Attention to features precedes attention to locations in visual search: evidence from electromagnetic brain responses in humans. Journal of Neuroscience, 24(8):1822-1832. 
Hopf, J. M., Luck, S. J., Boelmans, K., Schoenfeld, M. A., Boehler, C. N., Rieger, J., and Heinze, H.-J. (2006b). The neural site of attention matches the spatial scale of perception. Journal of Neuroscience, 26(13):3532-3540.

Janssen, P. and Shadlen, M. N. (2005). A representation of the hazard rate of elapsed time in macaque area LIP. Nature Neuroscience, 8(2):234-241.

Kandel, E. R., Schwartz, J. H., and Jessell, T. M., editors (2000). Principles of neural science. McGraw-Hill, New York, 4th edition.

Khayat, P. S., Spekreijse, H., and Roelfsema, P. R. (2006). Attention lights up new object representations before the old ones fade away. Journal of Neuroscience, 26(1):138-142.

Klemmer, E. T. (1956). Time uncertainty in simple reaction time. Journal of Experimental Psychology, 51(3):179-184.

Kreiman, G., Hung, C. P., Kraskov, A., Quiroga, R. Q., Poggio, T., and DiCarlo, J. J. (2006). Object selectivity of local field potentials and spikes in the macaque inferior temporal cortex. Neuron, 49(3):433-445.

Luck, S. J., Chelazzi, L., Hillyard, S. A., and Desimone, R. (1997). Neural mechanisms of spatial selective attention in areas V1, V2, and V4 of macaque visual cortex. Journal of Neurophysiology, 77(1):24-42.

Martínez-Trujillo, J. C. and Treue, S. (2004). Feature-based attention increases the selectivity of population responses in primate visual cortex. Current Biology, 14(9):744-751.

Maunsell, J. H. and Van Essen, D. C. (1983). Functional properties of neurons in middle temporal visual area of the macaque monkey. I. Selectivity for stimulus direction, speed, and orientation. Journal of Neurophysiology, 49(5):1127-1147.

Maunsell, J. H. R. (2004). The role of attention in visual cerebral cortex. In Chalupa, L. M. and Werner, J. S., editors, The Visual Neurosciences, pages 1538-1545. MIT Press, Cambridge, MA.

Maunsell, J. H. R. and Treue, S. (2006). Feature-based attention in visual cortex. Trends in Neurosciences, In Press.

McAdams, C. J. and Maunsell, J. H. (2000). Attention to both space and feature modulates neuronal responses in macaque area V4. Journal of Neurophysiology, 83(3):1751-1755. 
McAlonan, K., Cavanaugh, J., and Wurtz, R. H. (2006). Attentional modulation of thalamic reticular neurons. Journal of Neuroscience, 26(16):4444-4450.

McMains, S. A. and Somers, D. C. (2004). Multiple spotlights of attentional selection in human visual cortex. Neuron, 42(4):677-686.

Müller, N. G. and Kleinschmidt, A. (2003). Dynamic interaction of object- and space-based attention in retinotopic visual areas. Journal of Neuroscience, 23(30):9812-9816.

Newsome, W. T. and Pare, E. B. (1988). A selective impairment of motion perception following lesions of the middle temporal visual area (MT). Journal of Neuroscience, 8(6):2201-2211.

O’Connor, D. H., Fukui, M. M., Pinsk, M. A., and Kastner, S. (2002). Attention modulates responses in the human lateral geniculate nucleus. Nature Neuroscience, 5(11):1203-1209.

O'Craven, K. M., Downing, P. E., and Kanwisher, N. (1999). fMRI evidence for objects as the units of attentional selection. Nature, 401(6753):584-587.

Parker, A. J. and Newsome, W. T. (1998). Sense and the single neuron: probing the physiology of perception. Annual Review of Neuroscience, 21:227-277.

Posner, M. I. (1980). Orienting of attention. Quarterly Journal of Experimental Psychology, 32(1):3-25.

Posner, M. I. and Cohen, Y. (1984). Components of visual orienting. In Bouma, H. and Bouwhuis, D. G., editors, Attention and Performance X, pages 531-556. Erlbaum, Hillsdale, NJ.

Requin, J. and Granjon, M. (1969). The effect of conditional probability of the response signal on the simple reaction time. Acta Psychologica, 31(2):129-144.

Roelfsema, P. R., Lamme, V. A., and Spekreijse, H. (1998). Object-based attention in the primary visual cortex of the macaque monkey. Nature, 395(6700):376-381.

Sàenz, M., Buraĉas, G. T., and Boynton, G. M. (2002). Global effects of feature-based attention in human visual cortex. Nature Neuroscience, 5(7):631-632.

Saito, H., Yukie, M., Tanaka, K., Hikosaka, K., Fukada, Y., and Iwai, E. (1986). Integration of direction signals of image motion in the superior temporal sulcus of the macaque monkey. Journal of Neuroscience, 6(1):145-157. 
Salzman, C. D., Britten, K. H., and Newsome, W. T. (1990). Cortical microstimulation influences perceptual judgements of motion direction. Nature, 346(6280):174-177.

Treue, S. and Martínez-Trujillo, J. C. (1999). Feature-based attention influences motion processing gain in macaque visual cortex. Nature, 399(6736):575-579.

Treue, S. and Maunsell, J. H. (1999). Effects of attention on the processing of motion in macaque middle temporal and medial superior temporal visual cortical areas. Journal of Neuroscience, 19(17):7591-7602.

Tsao, D. Y., Freiwald, W. A., Tootell, R. B., and Livingstone, M. S. (2006). A cortical region consisting entirely of face-selective cells. Science, 311(5761):670-674.

Tsotsos, J. K., Culhane, S. M., Kei Wai, W. Y., Lai, Y., Davis, N., and Nuflo, F. (1995). Modelling visual attention via selective tuning. Artificial Intelligence, 78(1-2):507-545.

Ungerleider, L. G. and Mishkin, M. (1982). Two cortical visual systems. In Ingle, D. J., Goodale, M. A., and Mansfield, R. J. W., editors, Analysis of Visual Behavior, pages 549-586. MIT Press, Cambridge, MA.

Van Essen, D. C., Maunsell, J. H. R., and Bixby, J. L. (1981). The middle temporal visual area in the macaque: myeloarchitecture, connections, functional properties and topographic organization. Journal of Comparative Neurology, 199(3):293-326.

Watanabe, T., Harner, A. M., Miyauchi, S., Sasaki, Y., Nielsen, M., Palomo, D., and Mukai, I. (1998). Task-dependent influences of attention on the activation of human primary visual cortex. Proceedings of the National Academy of Sciences, 95(19):11489-11492.

Woldorff, M. G., Fox, P. T., Matzke, M., Lancaster, J. L., Veeraswamy, S., Zamarripa, F., Seabolt, M., Glass, T., Gao, J. H., Martin, C. C., and Jerabek, P. (1997). Retinotopic organization of early visual spatial attention effects as revealed by PET and ERPs. Human Brain Mapping, 5(4):280-286. 


\section{Curriculum Vitae}

\section{Steffen Katzner}

\section{Education}

01/02-07/06 Ph.D. student at the German Primate Center, Göttingen

Advisor: Prof. Dr. S.Treue

04/99-10/02 Diploma in Psychology, University of Tübingen

Thesis: Effects of stimulus and response probability on choice reaction time Advisor: Prof. Rolf Ulrich

04/99-09/01 M.Sc., Neural \& Behavioral Sciences, University of Tübingen

Thesis: Event-related attentional effects on sensory responses to moving stimuli in macaque area MT

Advisor: Prof. Stefan Treue

01/98-03/99 Research practical, Department of Psychology, University of Otago, New Zealand; Advisor: Prof. Jeff Miller

10/95-12/97 Undergraduate studies in Psychology, University of Wuppertal, Germany

10/91-09/93 Diploma in Business Administration \& Computer Science, Academy for Data Processing, Böblingen, Germany

\section{Working experience}

10/93-09/95 Systems programmer with Koch, Neff \& Oetinger, Stuttgart, Germany 


\title{
Journal publications
}

\author{
- $\quad$ Katzner, S., Busse, L., and Treue, S. (2006). Feature-based attentional inte- \\ gration of color and visual motion. Journal of Vision, 6(3):269-284. \\ - $\quad$ Busse, L. and Katzner, S. (2006). The time course of shifting visual atten- \\ tion. Journal of Neuroscience, 26(15):3885-3886. \\ Busse, L., Katzner, S., and Treue, S. (2006). Spatial and feature-based ef- \\ fects of exogenous cueing on visual motion processing. Vision Research, \\ 46(13):2019-2027.
}

\section{Published abstracts}

- $\quad$ Tillmann, C., Busse, L., Katzner, S. \& Treue, S. (2006). Attentional modulation of psychophysical tuning curves for direction of motion. European Conference on Visual Perception, St. Petersburg, Russia.

Katzner, S., Busse, L. \& Treue, S. (2005). Attending to the color in a motion signal: Evidence for object-based attentional effects in macaque area MT. Talk presented at the meeting of the Society for Neuroscience, Washington, DC.

- $\quad$ Busse, L., Katzner, S. \& Treue, S. (2005). Temporal dynamics of attentional modulation of neuronal responses in macaque area MT. Talk presented at the meeting of the Society for Neuroscience, Washington, DC.

Treue, S., Busse, L. \& Katzner, S. (2005). Behavioral benefits of signal anticipation in motion detection: improvement in perceptual sensitivity or adjustment of decision criterion? Poster presented at the meeting of the Society for Neuroscience, Washington, DC.

Busse, L., Katzner, S., Real, R. G. \& Treue, S. (2004). Time course of shifts of covert attention across features and space. Poster presented at the meeting of the Society for Neuroscience, San Diego.

Katzner, S., Busse, L. \& Treue, S. (2004). Dividing attention between color and motion: Feature-based effects of redundant targets. Poster presented at the meeting of the Society for Neuroscience, San Diego.

Katzner, S. Busse, L., Akcay, C. \& Treue, S. (2004). Processing visual 
motion and color: The effect of redundant targets. Poster presented at the meeting of the Cognitive Neuroscience Society, San Francisco.

Busse, L., Katzner, S. \& Treue, S. (2004). The effects of exogenous attention on the processing of visual motion. Poster presented at the meeting of the Cognitive Neuroscience Society, San Francisco.

Katzner, S. \& Treue, S. (2003). Dividing attention within or between hemifields: Effects on motion detection and discrimination thresholds. Poster presented at the meeting of the Society for Neuroscience, New Orleans.

Busse, L., Katzner, S. \& Treue, S. (2003). Spatial and feature-based effects of exogenous cueing on visual motion processing. Poster presented at the meeting of the Society for Neuroscience, New Orleans.

Katzner, S., Pieper, F. \& Treue, S. (2002). Effects of attention and contrast on visual motion detection and discrimination thresholds. Poster presented at the meeting of the Society for Neuroscience, Orlando.

Göttingen, 29th of May, 2006 